\title{
Studying the kinematics of the giant star-forming region 30 Doradus ${ }^{\star}$
}

\section{The data}

\author{
S. Torres-Flores ${ }^{1}$, R. Barbá ${ }^{1,2}$, J. Maíz Apellániz ${ }^{3}$, M. Rubio ${ }^{4}$, G. Bosch ${ }^{5}$, V. Hénault-Brunet ${ }^{6}$, and C. J. Evans ${ }^{7}$ \\ 1 Departamento de Física, Universidad de La Serena, Av. Cisternas 1200 Norte, La Serena, Chile \\ e-mail: storres@dfuls.cl \\ 2 Instituto de Ciencias Astronómicas, de la Tierra y del Espacio, Casilla 467, 5400 San Juan, Argentina \\ 3 Instituto de Astrofísica de Andalucía-CSIC, Glorieta de la Astronomía s/n, 18008 Granada, Spain \\ ${ }^{4}$ Departamento de Astronomía, Universidad de Chile, Casilla 36-D, Santiago, Chile \\ 5 Facultad de Ciencias Astronómicas y Geofísicas, Universidad Nacional de la La Plata, Paseo del Bosque s/n, 1900 La Plata, \\ Argentina \\ ${ }^{6}$ Institute for Astronomy, Royal Observatory Edinburgh, Blackford Hill, Edinburgh, EH9 3HJ, UK \\ 7 UK Astronomy Technology Centre, Royal Observatory Edinburgh, Blackford Hill, Edinburgh, EH9 3HJ, UK
}

Received 1 October 2012 / Accepted 30 April 2013

\section{ABSTRACT}

\begin{abstract}
We present high-quality VLT-FLAMES optical spectroscopy of the nebular gas in the giant star-forming region 30 Doradus. In this paper, the first of a series, we introduce our observations and discuss the main kinematic features of 30 Dor, as revealed by the spectroscopy of the ionized gas in the region. The primary data set consists of regular grid of nebular observations, which we used to produce a spectroscopic datacube of 30 Dor, centered on the massive star cluster R136 and covering a field-of-view of $10^{\prime} \times 10^{\prime}$. The main emission lines present in the datacube are from $\mathrm{H} \alpha$ and [NII] $\lambda \lambda 6548,6584$. The $\mathrm{H} \alpha$ emission-line profile varies across the region from simple single-peaked emission to complex, multiple-component profiles, suggesting that different physical mechanisms are acting on the excited gas. To analyse the gas kinematics we fit Gaussian profiles to the observed $\mathrm{H} \alpha$ features. Unexpectedly, the narrowest $\mathrm{H} \alpha$ profile in our sample lies close to the supernova remnant 30 Dor B. We present maps of the velocity field and velocity dispersion across 30 Dor, finding five previously unclassified expanding structures. These maps highlight the kinematic richness of 30 Dor (e.g. supersonic motions), which will be analysed in future papers.
\end{abstract}

Key words. ISM: bubbles - ISM: kinematics and dynamics - HII regions

\section{Introduction}

Giant H II regions (GHRs) are known as the site of massive young clusters which are rich in massive stars. The strong stellar winds and the evolution of these massive stars can disrupt the interstellar medium of these GHRs, resulting in the formation of expanding superbubbles. In the simplest scenario, the creation of superbubbles can be done by scaling the kinetic energy luminosity from the single-star models of Weaver et al. (1977). These models assume that shocked stellar winds dominate the dynamics of these bubbles. However, analysis of several superbubbles created by OB associations in the Large Magellanic Cloud (LMC; Oey 1996) and some extragalactic GHRs (Maíz-Apellániz \& Walborn 2001; MacKenty et al. 2000) reveals that such a model can not explain the observations. Recently, Lopez et al. (2011) studied the different feedback processes that are taking place in the 30 Doradus region in the LMC. They suggested that stellar feedback is the main process responsible in shaping the expansion of this GHR. In a different approach, Pellegrini et al. (2011) found that the large-scale structure of 30 Dor is dominated by a system of X-ray bubbles, which are in equilibrium between them. In spite of several studies to

^ Based on observations made with ESO Telescopes at the La Silla Paranal Observatory under programmes 072.C-0348 and 182.D-0222. investigate the origin of bubbles in GHRs, there is no consensus as to the mechanisms responsible, even in the well-studied case of 30 Dor.

A related, unsolved problem regarding GHRs is the origin of supersonic velocities seen in spatially-integrated nebular profiles. Given that supersonic motions should rapidly be dissipated, an energy source is needed to maintain them. Three possible sources exist: kinetic energy input from stellar winds and supernova explosions ( $\mathrm{SN})$, gravity, and photoionization. All of them can supply enough power to maintain the supersonic velocity dispersion but it is not clear which one(s) is/are responsible (Chu \& Kennicutt 1994; Tenorio-Tagle et al. 1993, 1996; Melnick et al. 1999). High-resolution spectroscopic data of nearby GHRs is necessary to disentangle these phenomena through the study of the kinematics of the warm gas. In this context, 30 Dor is one of the closest and most appropriate targets for undertaking a more detailed study.

The 30 Dor nebula is the largest H II region in the Local Group and the most powerful source of $\mathrm{H} \alpha$ emission in the LMC. At its core is the young massive cluster R136, host to a very large concentration of massive hot and luminous stars (see Crowther et al. 2010). Given all the information that can be extracted from 30 Dor, this object has been called the starburst "Rosetta Stone" (Walborn 1991). Due to its nature, 30 Dor has been the target of 
a range of multiwavelength studies (infrared, optical, ultraviolet and X-ray data) to try to disentangle its structure - both in terms of its stellar populations and the distribution of gas and dust. For instance, Bosch et al. (2009) searched for massive binary stars in the ionizing cluster of 30 Dor, finding a binary candidate rate of $50 \%$. These authors also derived the binary-corrected, virial mass of the cluster, which corresponds to $4.5 \times 10^{5} M_{\odot}$, suggesting that it could be a candidate for a future globular cluster. More recently, the VLT-FLAMES Tarantula Survey (VTFS, Evans et al. 2011) has opened a new window in the study of massive binary systems in 30 Dor (Sana et al. 2013), via the large number (over 800) of stars for which there are now high-quality spectroscopic data.

From imaging with the Hubble Space Telescope, Walborn et al. (2002) found some wind-blown cavities and several filamentary structures. While at X-ray wavelengths, Wang (1999) and Townsley et al. (2006a,b) found several X-ray bubbles and point sources in 30 Dor. In the case of the diffuse X-ray structures, which are spatially associated with high-velocity, optical emission-line clouds (Chu \& Kennicutt 1994), Townsley et al. (2006a) found that not every high-velocity feature displays bright X-ray emission. In this sense, optical kinematical analysis of the bright $\mathrm{X}$-ray regions is needed in order to understand this phenomenon.

The kinematics of the warm ionized gas in 30 Dor has also been well studied. For example, Smith \& Weedman (1972) used a Fabry-Perot instrument to map the [OIII] $\lambda 5007$ emission line, and suggested that the fast internal motions in the region are produced by the winds of WR stars. Chu \& Kennicutt (1994) and Melnick et al. (1999) both studied the kinematics of the ionized gas in 30 Dor, using echelle and long-slit spectroscopy, respectively. Chu \& Kennicutt (1994) found that 30 Dor displays very complex kinematics, with several fast expanding shells (that are coincident with extended X-ray sources) that can not be explained by stellar-wind models, and they suggested that SN remnants can solve that problem. Melnick et al. (1999) found complex $\mathrm{H} \alpha$ profiles in several regions of 30 Dor. They used their observations to study the mechanisms responsible for the linebroadening observed in the emission lines of $\mathrm{HII}$ regions and found a low-intensity broad component that explains the wings in the integrated profile of 30 Dor. Lastly, Redman et al. (2003) used echelle spectroscopy to study the gas kinematics in the outskirts of 30 Dor. These authors found some high-speed features, that they interpreted as shells formed by stellar winds and SNe.

These studies have each provided useful insights about the kinematics of 30 Dor, but the origin of superbubbles and the supersonic velocity dispersions in GHRs are still open questions. In fact, Melnick et al. (1999) suggested that it would be crucial to study the complete 30 Dor nebula at high spatial resolution. Thus, to address these problems, we have obtained highresolution spectroscopy of 30 Dor, using the FLAMES-Giraffe spectrograph on the Very Large Telescope (VLT) at the Paranal Observatory. Preliminary results from this programme have been published by Torres-Flores et al. (2011).

In this paper we present the data and discuss the general kinematics of 30 Dor, based on analysis of its nebular emissionlines. In future papers we will present analysis of: i) the width of the different emission lines and their contribution to the integrated emission-line profile of 30 Dor; ii) the kinematics of specific regions; iii) the wings detected in the integrated $\mathrm{H} \alpha$ profile of 30 Dor; iv) the connection between the kinematics of the ionized warm gas and the X-ray emission in this GHR. This paper is organized as follows. In Sect. 2 we present the data and the data-reduction process. In Sect. 3 we present the data analysis.
In Sect. 4 we show the general results obtained with the current data set. Finally, in Sect. 5 we summarize our findings.

\section{Observations}

\subsection{Data sets and fiber positioning}

To study the detailed kinematics of the ionized gas in 30 Dor we used the MEDUSA fiber mode of the VLT FLAMES-Giraffe spectrograph (Pasquini et al. 2002). Given the proximity of 30 Dor, FLAMES allows us to map, at high spectral and spatial resolution and high signal-to-noise, the main nebular emission lines from the gas, i.e., $\mathrm{H} \alpha$ and [NII] at 6548 and $6584 \AA$. In this regard, the multiplex of the MEDUSA observing mode enables us to sample a large number of points simultaneously across a relatively wide field-of-view; crucial when studying an extended region like 30 Dor. Given the complex morphology of 30 Dor, three different data sets are used to analyse the its kinematic features: a regular nebular grid, an irregular nebular grid (where the fibers were located in the brightest regions of 30 Dor), and finally a stellar grid. In the following we describe each data set in detail.

\subsubsection{The regular grid}

The observations of the regular nebular grid were carried out on December 3, 2003, using the HR14A Giraffe setting. This provided spectral coverage ranging from 6300 to $6691 \AA$, with a notional spectral resolving power of $R=17740$ at the central wavelength. We covered a field-of-view of $10^{\prime} \times 10^{\prime}$ centered on R136. Given that we were limited by the MEDUSA fiber separations, we used three different fiber configurations in offset positions to adequately sample our field-of-view; a total of 10 fields were observed, as summarised in Table 1 . These fields give a regular grid of $32 \times 30$ positions with a spatial sampling of $20^{\prime \prime}$, as shown in the top panel of Fig. 1, where the position of each fiber is indicated by a red circle. The aperture of each MEDUSA fiber corresponds to $1.2^{\prime \prime}$ on the sky $(\sim 0.3 \mathrm{pc})$.

Our configurations did not cover some positions given that two MEDUSA fibers were broken, producing some voids in the spatial sampling (see Fig. 1). Out of 960 fiber positions, 49 positions in the grid were not observed. We used nine fibers of the irregular grid to cover some of these missing positions (fibers nos. 11, 22, 23, 28, 31, 79, 100, 102 and 112). The positions of these fibers are indicated by blue numbered circles in the top panel of Fig. 1. In the case of fibers 28 and 31, we calculated an average of the two spectra and replaced this value at the corresponding missing position. The remaining 41 missing positions were filled with the average of the eight closest (and spatially linked) spectra. In this sense, we caution the reader that the spectra visible in the positions of the missing fibers do not represent the real emission at these locations (see Fig. 1).

The centers of the different configurations, setups, and exposure times used to observe 30 Dor are listed in Table 1. Given the high surface-brightness displayed by 30 Dor, two sets of observations were taken to avoid saturation effects. In the first instance we took three exposures of $60 \mathrm{~s}$ and then we took two exposures of $600 \mathrm{~s}$. At the end, we co-added the two observations of $600 \mathrm{~s}$. The fiber positions for the regular grid are listed in Table A.1; when a fiber was taken from the irregular grid (as described above), it is labelled as "IG".

Thus, from the regular grid we have obtained 911 equallyspaced spectra across an area of $10^{\prime} \times 10^{\prime}$ centered on R136. 
Table 1. VLT-FLAMES/MEDUSA observing log for the regular nebular grid.

\begin{tabular}{lcccc}
\hline \hline Field & Coordinates & Setup & $N$. Fibers & Exp. [s] \\
\hline 1 & $05: 38: 42.0,-69: 05: 40.0$ & HR14, Medusa1 & 101 & $2 \times 600$ \\
2 & $05: 38: 45.7,-69: 05: 40.0$ & HR14, Medusa1 & 101 & $2 \times 600$ \\
3 & $05: 38: 45.7,-69: 05: 20.0$ & HR14, Medusa1 & 76 & $2 \times 600$ \\
4 & $05: 38: 42.0,-69: 05: 20.0$ & HR14, Medusa1 & 101 & $2 \times 600$ \\
5 & $05: 38: 42.0,-69: 05: 40.0$ & HR14, Medusa2 & 98 & $2 \times 600$ \\
6 & $05: 38: 45.7,-69: 05: 40.0$ & HR14, Medusa2 & 98 & $2 \times 600$ \\
7 & $05: 38: 45.7,-69: 05: 20.0$ & HR14, Medusa2 & 98 & $2 \times 600$ \\
8 & $05: 38: 42.0,-69: 05: 20.0$ & HR14, Medusa2 & 98 & $2 \times 600$ \\
9 & $05: 38: 42.0,-69: 09: 00.0$ & HR14, Medusa1 & 71 & $2 \times 600$ \\
10 & $05: 38: 42.0,-69: 08: 40.0$ & HR14, Medusa1 & 69 & $2 \times 600$ \\
\hline
\end{tabular}

With that data in-hand, we sorted all the spectra in right ascension and declination. Given that each fiber gave us spectral information for each equally-spaced point, the regular grid enabled us to produce a high-resolution spectroscopic datacube of 30 Dor, spanning 6300 to $6691 \AA$. This datacube can provide us spatial and spectral information simultaneously, where the spatial information depends on the fiber positions and the spectral information depends on the wavelengths covered by the spectra. For instance, a slice of this datacube centered at $\mathrm{H} \alpha$ can give us a monochromatic image of 30 Dor, albeit limited in spatial resolution to $20^{\prime \prime}$ by our fiber separation. Despite this fact, the datacube give us an excellent means by which to investigate the kinematics of the large expanding structures in 30 Dor. To illustrate the spectral coverage and primary nebular emission features in the spectra, the integrated spectrum (summed from all spectra in the datacube) is shown in Fig. 2. Given that the most intense emission lines in Fig. 2 correspond to $\mathrm{H} \alpha$ and [NII] $\lambda 6584$, we have derived two sub-datacubes centered in these lines, where these sub-cubes cover a range of $500 \mathrm{~km} \mathrm{~s}^{-1}$ in velocity.

In this paper we focus on analysis of the regular grid, but we now briefly introduce the other two data sets as they will be employed in the future studies.

\subsubsection{The irregular grid}

Additional observations were obtained in the same observing run as the regular grid. This "irregular grid" of MEDUSA positions was selected to observe the brightest nebular regions of 30 Dor, which can be associated with photodissociation regions which lie between the bright nebular and molecular phases. The same high-resolution setup (i.e. HR14A) was used as the regular grid. To reach a high signal-to-noise ratio, nine exposures (each of $600 \mathrm{~s}$ ) were obtained with this configuration; the positions are shown in the middle panel of Fig. 1 (red circles). The red rectangle indicates the field-of-view covered by the regular nebular grid; as can be seen most of the fibers were located across the filamentary structure of 30 Dor. Each fibre position is listed in Table A.2.

\subsubsection{The stellar grid}

The observations of the stellar grid were obtained as part of the VFTS (Evans et al. 2011). This multi-epoch spectral survey aims to detect massive binary systems and determine their nature and evolution. The VFTS also aims to study the properties of stellar winds and rotational mixing in O-type stars. To carry out these studies, the survey has observed 800 massive stars, as shown in the bottom panel of Fig. 1. Of relevance here, the red-optical VFTS data were observed with the HR15N Giraffe setup, which has a spectral coverage ranging from 6442 to $6817 \AA$, at a spectral resolving power of $R=16000$. The HR $15 \mathrm{~N}$ setup provides the additional nebular diagnostic of the [SII] lines at $\lambda \lambda 6716$, $6731 \AA$, which are not covered by the HR14A observations. Given the large spatial coverage of the VFTS survey, these data can provide additional insights in the study of the kinematics of 30 Dor.

\subsection{Data reduction}

Reduction of the 2003 data was performed with the ESO pipeline GASGANO and EsoRex software. We observed three bias and flats, which were combined to correct our observations. The wavelength calibration was performed by using the ThAr calibration lamp, from which the instrumental resolution was measured to have a FWHM of $0.4 \AA$, which corresponds to $R=$ 16400 at $\mathrm{H} \alpha$. All the spectra were corrected to the heliocentric rest frame.

We have compared our processed data with the data available in the Giraffe archive ${ }^{1}$. By visual inspection, the two reductions are in good agreement. Cosmic rays were removed using the task CRREJECT in IRAF. Once the data were bias/flat-field corrected and wavelength calibrated, we removed the continuum emission present in the spectra by fitting a polynomial (using the IRAF CONTINUUM task). In a few cases in the regular grid, the fibers lie close to some stars; for these we use a high-order polynomial to remove the continuum emission. To remove the sky emission lines, we fit Gaussians to one of the lowest intensity spectra. This allowed us to create a template of sky lines, which were removed from all the spectra.

\section{Analysis}

Past efforts to understand the internal kinematics of H II regions have employed profile widths of their emission lines as a diagnostic (e.g., Chu \& Kennicutt 1994). Following a similar approach, we use the $\mathrm{H} \alpha$ line from our observations to characterize the kinematics of the gas, as it presents the highest signal-tonoise ratio and can be easily resolved into different components in our data. To obtain the line widths we fitted Gaussians automatically to each observed $\mathrm{H} \alpha$ profile. In several cases a single Gaussian does not represent the real shape of the multiple profiles that can be seen in this star-forming region (e.g., Melnick et al. 1999). However, this exercise provides some insight about the general behaviour of the $\mathrm{H} \alpha$ emission.

We used the MPFIT package in IDL (Markwardt 2009) to obtain the center, peak intensity and the observed width $\left(\sigma_{\mathrm{obs}}\right.$,

1 http://giraffe-archive.obspm.fr/ 

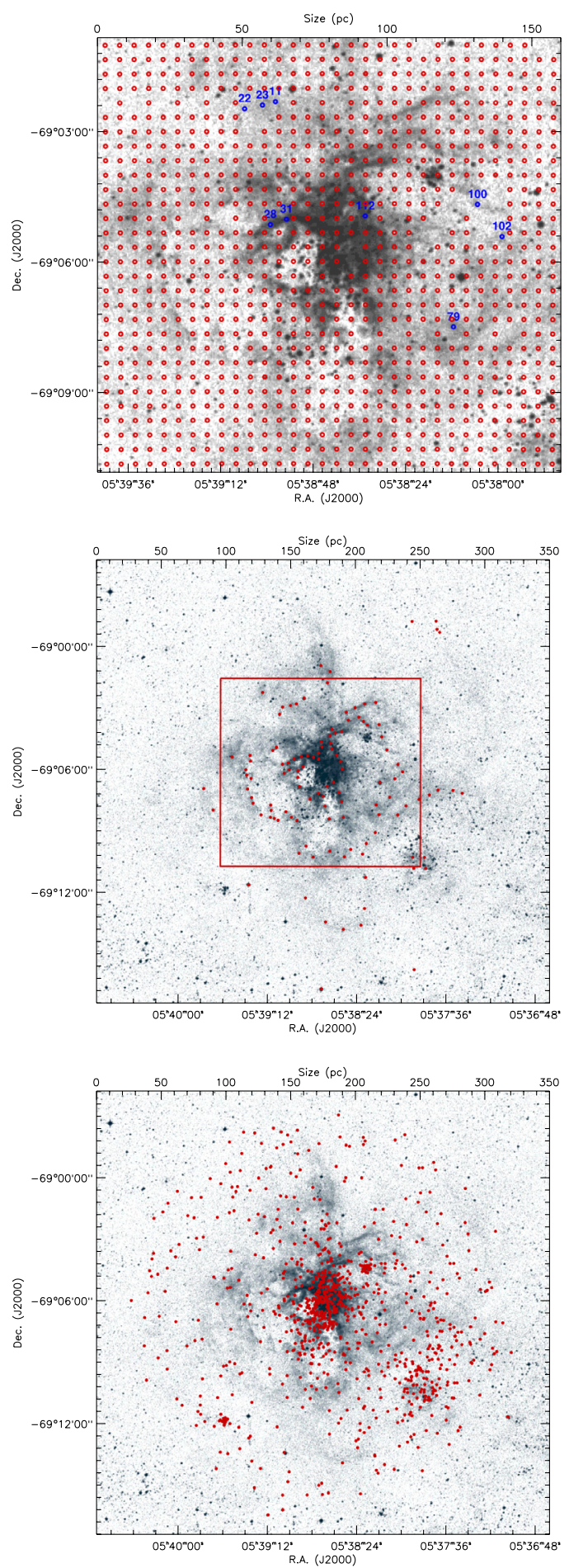

Fig. 1. Top panel: GIRAFFE/MEDUSA regular grid overlaid on an optical Digital Sky Survey image of 30 Dor. The spatial separation between each fiber corresponds to $20^{\prime \prime}$ ( $\left.\sim 5 \mathrm{pc}\right)$. Middle panel: MEDUSA irregular grid. Bottom panel: MEDUSA stellar grid (VFTS survey). The field-of-view on the sky shown in the lower two panels is larger than in the top panel to show all the fibers available. The red rectangle in the middle panel indicates the region covered by the regular grid.

uncorrected by instrumental and thermal broadening) of each emission line. Thereafter, where relevant, we fitted multiple Gaussians to the observed profiles using the PAN package ${ }^{2}$, which enabled us to select the number of components for each

\footnotetext{
2 http://ifs.wikidot.com/pan
}

observed profile ${ }^{3}$. This kind of analysis is necessary given the complexity of the emission lines in 30 Dor. For example, Melnick et al. (1999) showed that more than three Gaussian components are needed to reproduce some of the observed profiles. We note that this kind of exercise is (to some degree) arbitrary, given that in several cases we can not resolve two superposed $\mathrm{H} \alpha$ profiles (which lie at the same radial velocity, for example), which we would fit with just one Gaussian.

The width of the observed $\mathrm{H} \alpha$ profiles take into account the instrumental $\left(\sigma_{\text {in }}\right)$ and thermal $\left(\sigma_{\text {th }}\right)$ widths. The former value depends on the resolution of the instrument, while the latter depends on the thermal motions of the Hydrogen. To obtain a corrected value for the width of the $\mathrm{H} \alpha$ emission line, we have subtracted $\sigma_{\text {in }}$ and $\sigma_{\text {th }}$ from $\sigma_{\text {obs }}$ as follows: $\sigma^{2}=\sigma_{\text {obs }}^{2}-\sigma_{\text {in }}^{2}-\sigma_{\text {th }}^{2}$, where $\sigma$ represents the true width of the $\mathrm{H} \alpha$ line. As discussed in Sect. 2.2, $\sigma_{\text {in }}$ was derived from the calibration lamp exposures and has a value of $\sigma_{\text {in }}=7.8 \mathrm{~km} \mathrm{~s}^{-1}$. For $\sigma_{\text {th }}$ we assume hydrogen gas at an electronic temperature of $10^{4} \mathrm{~K}$, from which $\sigma_{\text {th }} \sim 9.1 \mathrm{~km} \mathrm{~s}^{-1}$.

\section{Results}

\subsection{General view of the 30 Dor spectroscopic datacube}

To visualize the complex morphology of the ionized emission in 30 Dor, in Fig. 3 we have plotted the $\mathrm{H} \alpha$ profile of each fiber from the regular grid (normalized by the peak of the intensity in each case) over an $\mathrm{H} \alpha$ image obtained with the EMMI instrument at the La Silla Observatory ${ }^{4}$.

In Fig. 3 it is possible to correlate the high-resolution spectral information with features seen in the image. The $\mathrm{H} \alpha$ profiles in many instances are extremely complex. For example, in the cavity located $1^{\prime}$ to the east of $\mathrm{R} 136$, the $\mathrm{H} \alpha$ profiles clearly display multiple components. In fact, the fiber located at the position $\alpha=05^{\mathrm{h}} 38^{\mathrm{m}} 53.21^{\mathrm{s}} \delta=-69^{\circ} 05^{\prime} 59.89^{\prime \prime}(\mathrm{J} 2000)$ displays at least five components (see the last panel of Fig. 7). In general, most of the multiple $\mathrm{H} \alpha$ profiles are located in the regions where expanding shells have been previously identified (see Chu \& Kennicutt 1994). On the other hand, Fig. 3 shows that the most intense $\mathrm{H} \alpha$ emitting regions in 30 Dor (based on its EMMI image) display simple and narrow profiles. In several cases, these bright regions are associated with photodissociation regions (PDRs), which can halt some expanding structures, producing simple profiles. In addition, Fig. 3 shows that some low-intensity regions display simple profiles (for example, in the north-east region), which suggests that no expanding structures lie at that location. By inspecting Fig. 3 it is also possible to identify low-/high-velocity components. For instance, at $\alpha=05^{\mathrm{h}} 37^{\mathrm{m}} 49.66^{\mathrm{s}} \delta=-69^{\circ} 02^{\prime} 39.91^{\prime \prime}$ we find a low-intensity component having an approaching velocity larger than $200 \mathrm{~km} \mathrm{~s}^{-1}$ compared to the systemic value.

Given the kinematic richness of 30 Dor, we have derived a velocity-sliced view of the datacube, centered on the $\mathrm{H} \alpha$ and [NII] $\lambda 6584$ lines. In Fig. 4 we show 20 frames of the datacube. The systemic (zero) velocity was defined by the Gaussian fit to the integrated $\mathrm{H} \alpha$ line (i.e. from Fig. 2), in which the line-center was $6568.65 \AA$, equivalent to $v_{\mathrm{r}}=267.4 \mathrm{~km} \mathrm{~s}^{-1}$ (where the rest

\footnotetext{
3 For consistency, we have checked the results derived from MPFIT and PAN in the case when just one Gaussian is fitted, finding the same results.

4 This image was taken on December 2002, under the program 70.C-0435(A), using the filter Ha\#596, with a total exposure time of $1340 \mathrm{~s}$.
} 
S. Torres-Flores et al.: Studying the kinematics of the giant star-forming region 30 Doradus. I.

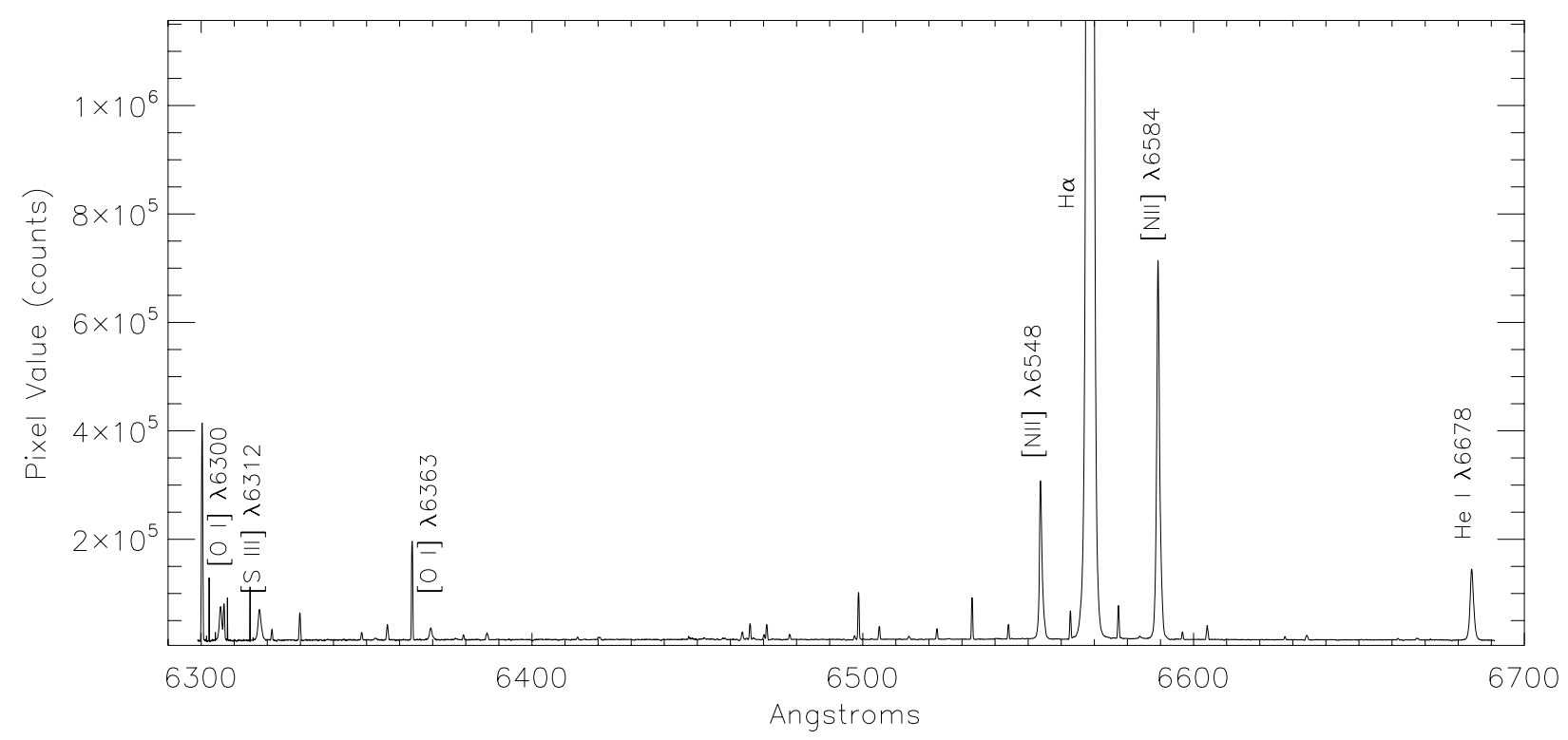

Fig. 2. Integrated nebular spectrum of 30 Dor obtained from summing all of the spectra from the regular grid. The most intense emission line is $\mathrm{H} \alpha$, but the intensity axis in the plot is truncated to show the low-intensity emission lines.

wavelength of the $\mathrm{H} \alpha$ emission line was taken from Hirata \& Horaguchi 1995) and the velocity interval between each frame was $25.1 \mathrm{~km} \mathrm{~s}^{-1}$. In this case, negative and positive velocities imply radially approaching and receding velocities, respectively.

By inspecting the first four frames of Fig. 4, we can see several high-velocity components with respect to the mean velocity of 30 Dor, especially in the neighborhood of the Hodge 301 cluster (the high velocity components in the first and second frames). These components reach approaching velocities larger than $230 \mathrm{~km} \mathrm{~s}^{-1}$. By inspecting the datacube we found several lowintensity components that present velocities even larger than $250 \mathrm{~km} \mathrm{~s}^{-1}$. For instance, Redman et al. (2003) found several discrete high-speeds knots in 30 Dor, which have velocities of $\pm 200 \mathrm{~km} \mathrm{~s}^{-1}$. In the last frames of Fig. 4 we also note several high-velocity components - some of these can be associated with the expanding structure \#5 from Chu \& Kennicutt (1994, their Fig. 1c).

Another interesting feature that appears in Fig. 4 is the detection of shell-like structures which are visible across all the observed field of 30 Dor (see Sect. 4.4). These structures appear as small voids that increase in size as we move from the blue to the red side of the $\mathrm{H} \alpha$ line. It is interesting to note that bluewards of the systemic $\mathrm{H} \alpha$ emission the shells are clearly defined, while they are not seen in the frames redwards of the central velocity. Internal extinction produced by dust can produce this signature. Also, molecular clouds located at these positions could hide the red side of the $\mathrm{H} \alpha$ emission.

In Fig. 5, we show a similar velocity-sliced view, but now centered on the [NII] $\lambda 6584$ emission line. As in the previous case, the systemic (zero) velocity was derived from the Gaussian fit to the integrated [NII] $\lambda 6584$ line, which gives a radial velocity of $v_{\mathrm{r}}=266.1 \mathrm{~km} \mathrm{~s}^{-1}(6589.29 \AA)$. As to be expected from the reduced intensity of the [NII] line compared to $\mathrm{H} \alpha$, most of the emission is detected at lower expanding velocities than seen in the $\mathrm{H} \alpha$ map. While there is a clear correlation between the morphology of the shell-like structures detected in both lines, inspection of Figs. 4 and 5 shows that several $\mathrm{H} \alpha$ and [NII] $\lambda 6584$ highvelocity components do not correlate spatially, which suggests that different physical processes are exciting the gas at these locations. For example, in Fig. 6 we show a high-velocity component in the [NII] $\lambda 6584$ line (black solid line) which presents a strong intensity with respect to their $\mathrm{H} \alpha$ counterpart $(\alpha=$ $\left.05^{\mathrm{h}} 38^{\mathrm{m}} 41.97^{\mathrm{s}} \delta=-69^{\circ} 04^{\prime} 40.04^{\prime \prime}\right)$. In the same figure, we see the spectrum of the next observed fiber (red dashed line), which present a high-velocity component in $\mathrm{H} \alpha$ but with no [NII] $\lambda 6584$ emission $\left(\alpha=05^{\mathrm{h}} 38^{\mathrm{m}} 41.96^{\mathrm{s}} \delta=-69^{\circ} 05^{\prime} 00.03^{\prime \prime}\right)$. We note that in this comparison we are using two adjacent observed fibers. These points all help to illustrate the capabilities of these data in disentangling the complex structures of 30 Dor.

\subsection{Complexity of the Ho profiles in 30 Doradus}

\subsubsection{From single to multiple profiles}

The wide variety of $\mathrm{H} \alpha$ profiles in the data are illustrated by the examples in Fig. 7, which show the four narrowest and broadest profiles (upper and lower panels, respectively). All these profiles correspond to those observed and not to the averaged profiles described in Sect. 2.1.1. A single Gaussian was fitted to each observed profile (as described in Sect. 3), with the coordinates of the fiber position and $\sigma_{\mathrm{obs}}$ derived from the fitting process shown in the upper right of each panel. In this analysis, the center of the Gaussian fit gives the overall systemic radial velocity at the position of the fiber, while the width of this fit gives the velocity dispersion of the ionized gas, which must be corrected by the instrumental and thermal widths.

The narrowest $\mathrm{H} \alpha$ profile has a $\sigma_{\mathrm{obs}}=14.3 \mathrm{~km} \mathrm{~s}^{-1}$ (upper left-hand panel in Fig. 7), which corresponds to $\sigma=7.8 \mathrm{~km} \mathrm{~s}^{-1}$ once corrected for $\sigma_{\text {th }}$ and $\sigma_{\text {in }}$. This profile is located in the south-west of the nebula, which is ionized by the LH99 stellar association (Lucke \& Hodge 1970) and is also the location of the SN remnant 30 Dor B (Danziger et al. 1981). Two low-intensity components can also be seen in the profile, and these could be associated with an expanding structure. Nonetheless, the main structure of this profile is symmetric and can be well-fitted by a single Gaussian (red dashed line in Fig. 7). The other three narrow $\mathrm{H} \alpha$ profiles shown in Fig. 7 display a few low-intensity components, but the main part of the profiles are also well-fitted by a single Gaussian. 


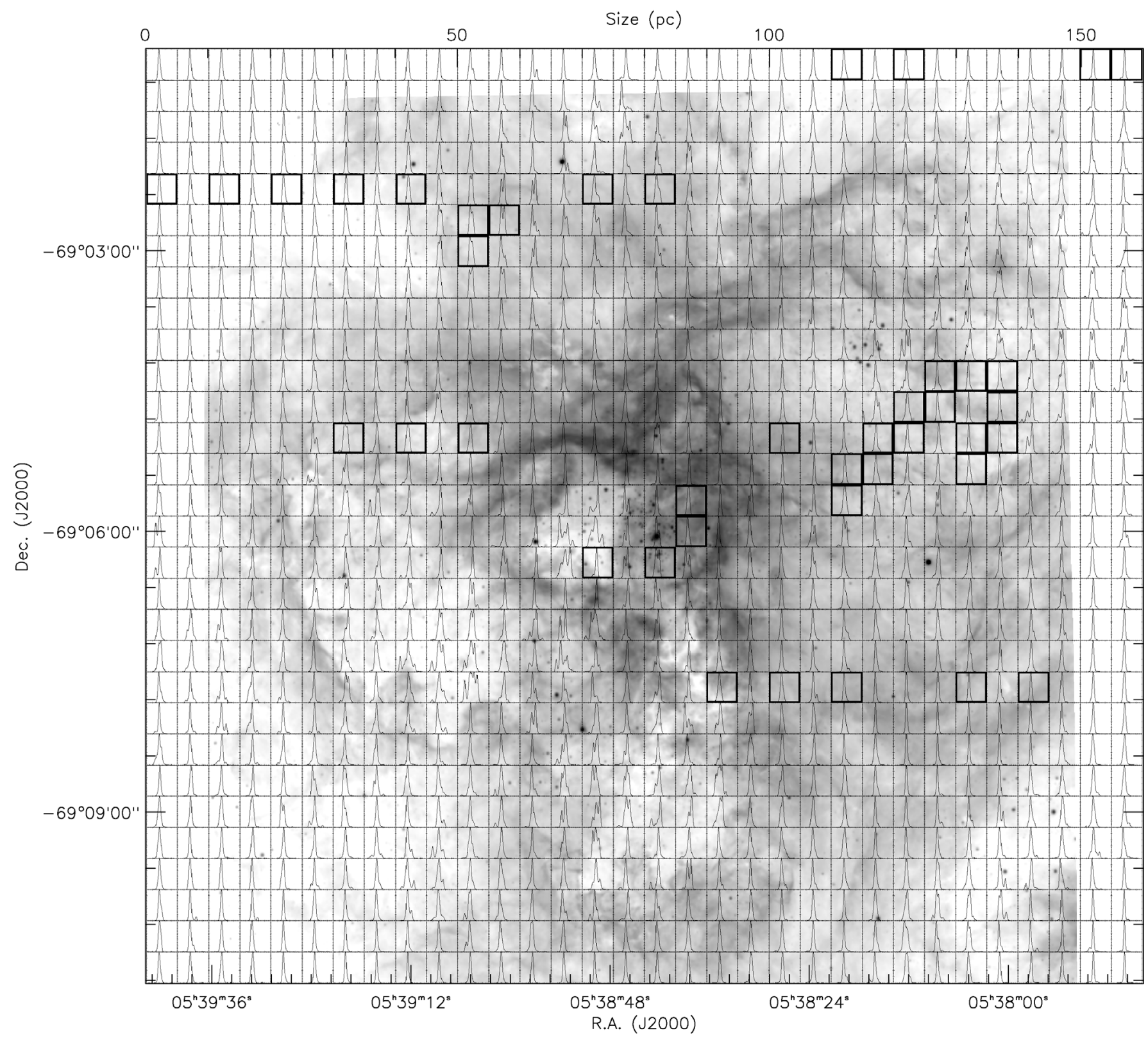

Fig. 3. ESO/La Silla H $\alpha$ image of 30 Dor (taken with the EMMI instrument), on which we have over-plotted the H $\alpha$ profile from each fiber of the regular nebular grid. Each small box is $20^{\prime \prime} \times 20^{\prime \prime}(5 \mathrm{pc} \times 5 \mathrm{pc})$, with the fibers located at the center of each box. Thick boxes indicate the positions for which we have interpolated the spectra (see Sect. 2.1.1).

In contrast, the broad profiles shown in Fig. 7 clearly display multiple strong components. As expected, these profiles can not be fit by a single Gaussian and their measured widths are the result of a coarse fit that takes into account all the components. All of these broad, multiple profiles lie in shell-like structures. It is interesting to note that the third broad profile shown in Fig. 7 lies just $20^{\prime \prime}$ (equivalent to $\sim 5 \mathrm{pc}$ ) from the narrow profile located at $\alpha=05^{\mathrm{h}} 38^{\mathrm{m}} 56.945^{\mathrm{s}} \delta=-69^{\circ} 06^{\prime} 19.940^{\prime \prime}$. This shows the extremely complex structure of 30 Dor even at small spatial scales.

\subsubsection{Multiple Gaussian fits: a few examples}

To determine the line-broadening mechanism in 30 Dor, Melnick et al. (1999) fitted multiple Gaussian components to the observed $\mathrm{H} \alpha$ profiles of different regions. They found that a broad, low-intensity component was necessary to reproduce the wings of all the observed $\mathrm{H} \alpha$ profiles. As noted earlier, we have performed multiple Gaussian fits to a subset of profiles using the PAN package. In particular, we have examined the two narrowest and broadest $\mathrm{H} \alpha$ profiles from single-component fits on the FLAMES data (see Fig. 7), with results of these fits shown in Fig. 8. In each panel of the figure the red (dashed) lines indicate the different Gaussian components used in the fits; the coordinates of the fiber position and the $\sigma_{\text {obs }}$ of each fitted component are given in the upper right of the panels. In Fig. 8 we also plot the residuals of the fits, i.e. the difference between the fitted and the observed profile, in which the intensity axis was limited to $\pm 5 \%$ of the peak of the observed profile.

For the narrowest $\mathrm{H} \alpha$ profile in the data (upper left in Fig. 8), we have fitted three Gaussian components. The two lowintensity components detected at this position present widths smaller than $\sigma_{\mathrm{obs}} \sim 26 \mathrm{~km} \mathrm{~s}^{-1}$. The strongest component at this 
S. Torres-Flores et al.: Studying the kinematics of the giant star-forming region 30 Doradus. I.
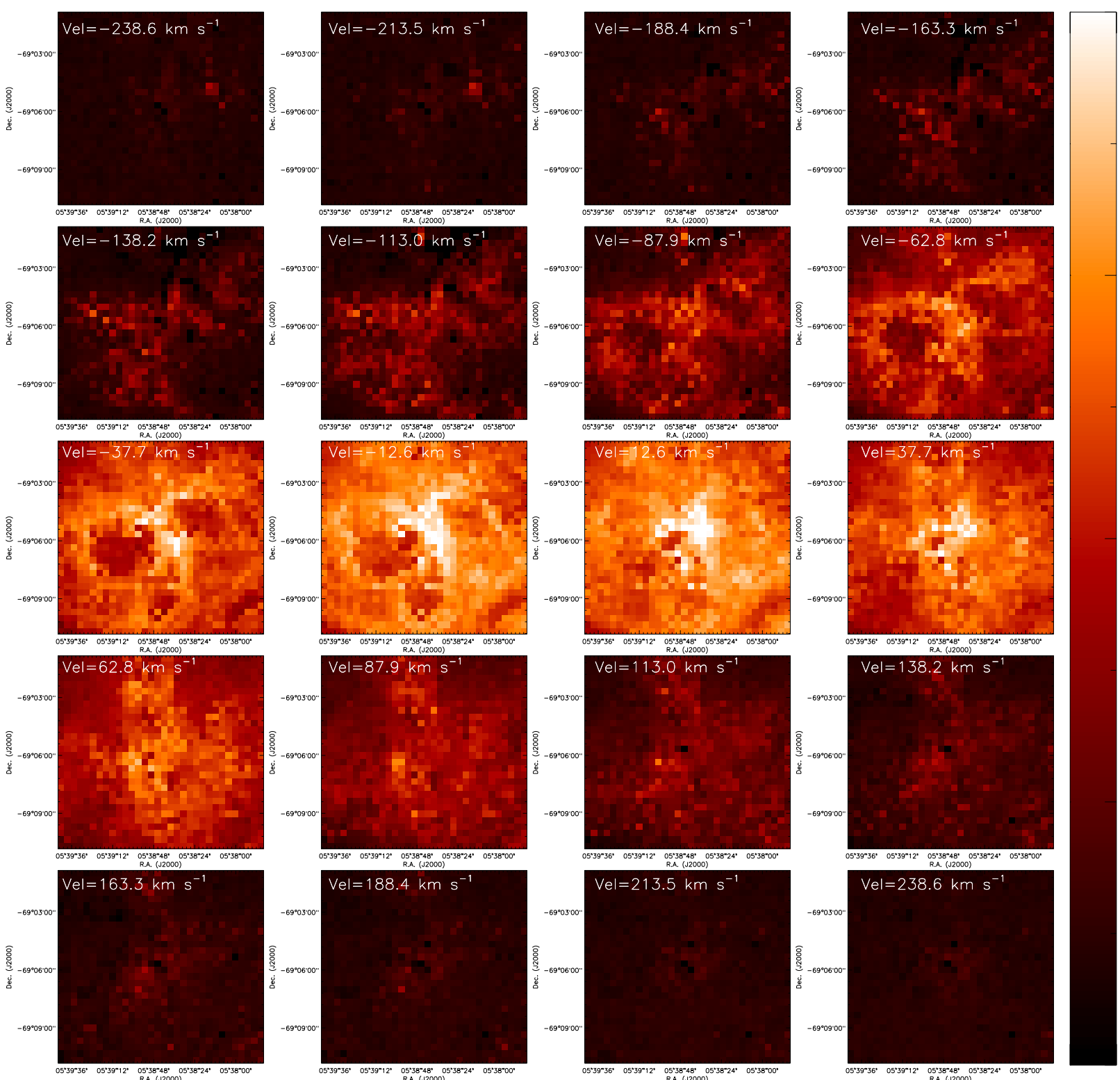

Fig. 4. Velocity-sliced view of the 30 Dor datacube centered on the $\mathrm{H} \alpha$ line. The velocity with respect to the center of the $\mathrm{H} \alpha$ line is shown in the upper left-hand corner of each panel (negative and positive velocities imply radially approaching and receding velocities, respectively). In this figure, a logarithmic scale is used for the intensity of each map.

position has a corrected width of $\sigma=6.1 \mathrm{~km} \mathrm{~s}^{-1}$, i.e., lower than the value obtained by fitting just one Gaussian $\left(\sigma=7.8 \mathrm{~km} \mathrm{~s}^{-1}\right.$, from Fig. 7). By inspecting the residual of these fits, no lowintensity broad component is necessary to explain the observed profile (the residuals are negligible compared with the observed emission). This is of wider interest as this $\mathrm{H} \alpha$ profile is located in 30 Dor B, associated with a SN remnant (where we can expect a complex kinematic). In the case of the second-narrowest profile, we have fitted three Gaussian components. The central, most intense feature is fit with a narrow component with a corrected width of $7.8 \mathrm{~km} \mathrm{~s}^{-1}$, together with two broader, lowintensity components with corrected widths of $19.2 \mathrm{~km} \mathrm{~s}^{-1}$ and $31.8 \mathrm{~km} \mathrm{~s}^{-1}$.

The scenario is quite different when multiple Gaussians are fitted to the broadest and more complex $\mathrm{H} \alpha$ profiles. In the lower left-hand panel of Fig. 8, we show that at least six components are necessary to reproduce the observed emission line at that position. The broadest component has a corrected width of $\sigma=$ $28.5 \mathrm{~km} \mathrm{~s}^{-1}$. As in the previous cases, the residual is negligible compared with the intensity of the profiles. Finally, in the lower right-hand panel of Fig. 8 we show another spectrum which requires six components to fit the observations. In this case, the broadest component has a corrected width of $\sigma=48.8 \mathrm{~km} \mathrm{~s}^{-1}$. It is interesting to note that four of the six broad components used in the Gaussian fits shown in the lower panel of Fig. 8 display supersonic widths (once corrected by $\sigma_{\text {th }}$ and $\sigma_{\text {ins }}$ ). Despite other Gaussian components can be fitted in these wide profiles, our results suggest the presence of a highly turbulent gas at these locations.

We note that Melnick et al. (1999) found that most of the $\mathrm{H} \alpha$ profiles in their analysis required the presence of a lowintensity broad component with $\sigma=45 \mathrm{~km} \mathrm{~s}^{-1}$, Moreover, this broad component was required to fit their integrated $\mathrm{H} \alpha$ profile of 30 Dor, centered at the radial velocity of this GHR. This 

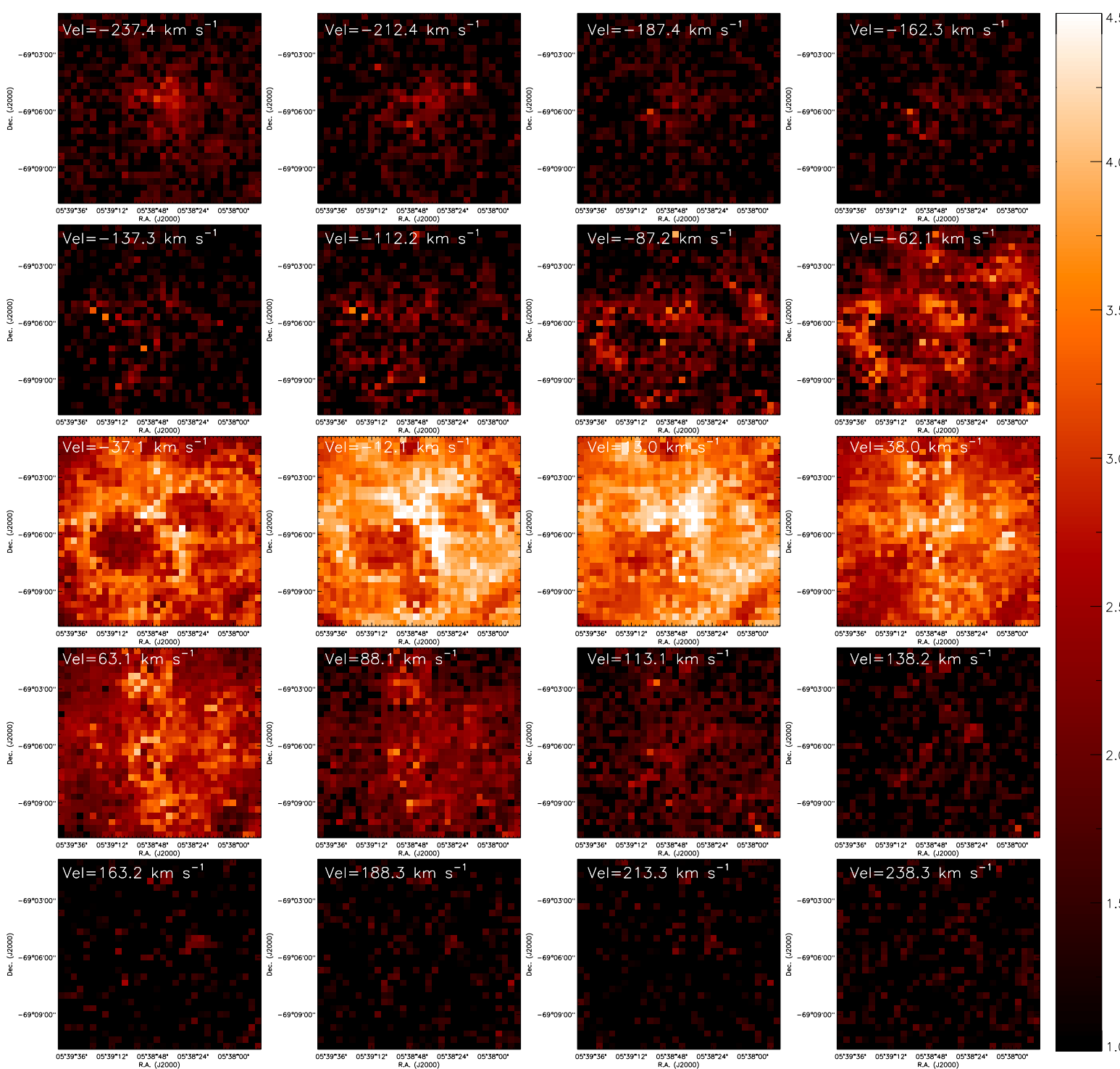

Fig. 5. Velocity-sliced view of the 30 Dor datacube centered on the [NII] $\lambda 6584$ line. The velocity with respect to the center of the [NII] $\lambda 6584$ line is shown in the upper left-hand corner of each panel (negative and positive velocities imply radially approaching and receding velocities, respectively). In this figure, a logarithmic scale is used for the intensity of each map.

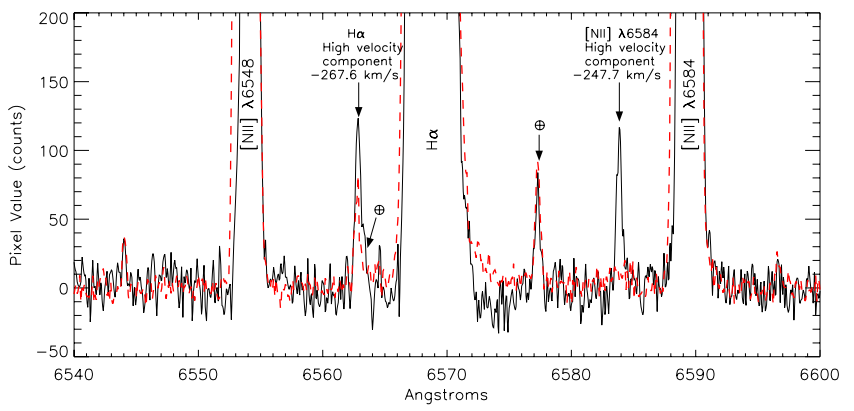

Fig. 6. Example of a high-velocity component seen in the [NII] 16584 line. The black solid and red dashed lines correspond to the spectra of two contiguous fibers on the sky. The black solid spectrum shows a [NII] high velocity component which is intense in comparison with its $\mathrm{H} \alpha$ counterpart. This effect is not detected in the red dashed spectrum.

component was not identified in the two narrowest profiles observed by FLAMES, nor in the second broadest profile (shown in
Fig. 8). In fact, the broad components that we have identified are not at the systemic radial velocity of the 30 Dor gas; the origin of the broad component in 30 Dor, if real, is still a mystery.

\subsubsection{The integrated $\mathrm{H} \alpha$ profile of 30 Doradus}

Fits to the integrated $\mathrm{H} \alpha$ profile (from Fig. 2) are shown in Fig. 9. For a single-component fit we found a corrected width of $\sigma=26.5 \mathrm{~km} \mathrm{~s}^{-1}\left(\sigma_{\mathrm{obs}}=29.1 \mathrm{~km} \mathrm{~s}^{-1}\right)$. Note from the figure that the blue and red wings of the integrated profile can not be fitted by one component - a second, broader component is required, as shown in the lower panel of Fig. 9. By using narrow, high-intensity and broad, low-intensity components, we obtain a much better fit to the observed $\mathrm{H} \alpha$ profile of 30 Dor. In this case, the narrow and the broad components have corrected widths of $\sigma=21.2 \mathrm{~km} \mathrm{~s}^{-1}\left(\sigma_{\mathrm{obs}}=24.4 \mathrm{~km} \mathrm{~s}^{-1}\right)$ and $\sigma=47.6 \mathrm{~km} \mathrm{~s}^{-1}$ $\left(\sigma_{\mathrm{obs}}=49.1 \mathrm{~km} \mathrm{~s}^{-1}\right)$, respectively. Our result for the narrow component is in good agreement with the corrected width from Melnick et al. $\left(1999, \sigma=22 \mathrm{~km} \mathrm{~s}^{-1}\right)$ from the data of Chu \& Kennicutt (1994); our broader component is marginally wider 
S. Torres-Flores et al.: Studying the kinematics of the giant star-forming region 30 Doradus. I.
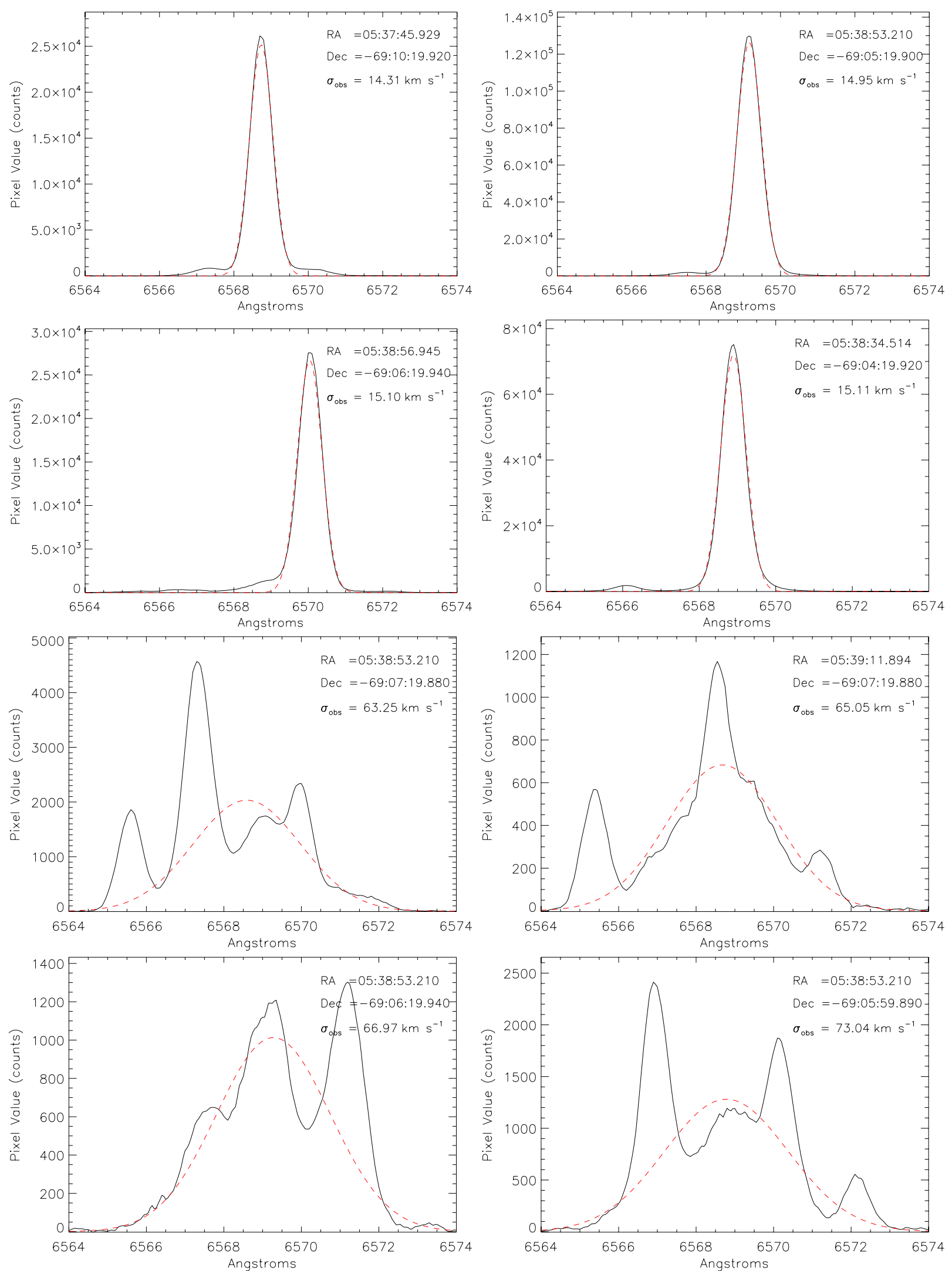

Fig. 7. Examples of the different $\mathrm{H} \alpha$ morphologies detected in 30 Dor. In the first four panels we show the narrowest $\mathrm{H} \alpha$ profiles found in this study. In contrast, the last four panels show the broadest velocities arising from single-profile fits (which are clearly a consequence of multiple components in the observed lines). In each panel we note the coordinates of the observation and $\sigma_{\mathrm{obs}}$ of the single-profile Gaussian fit (indicated by the overplotted red dashed lines). 

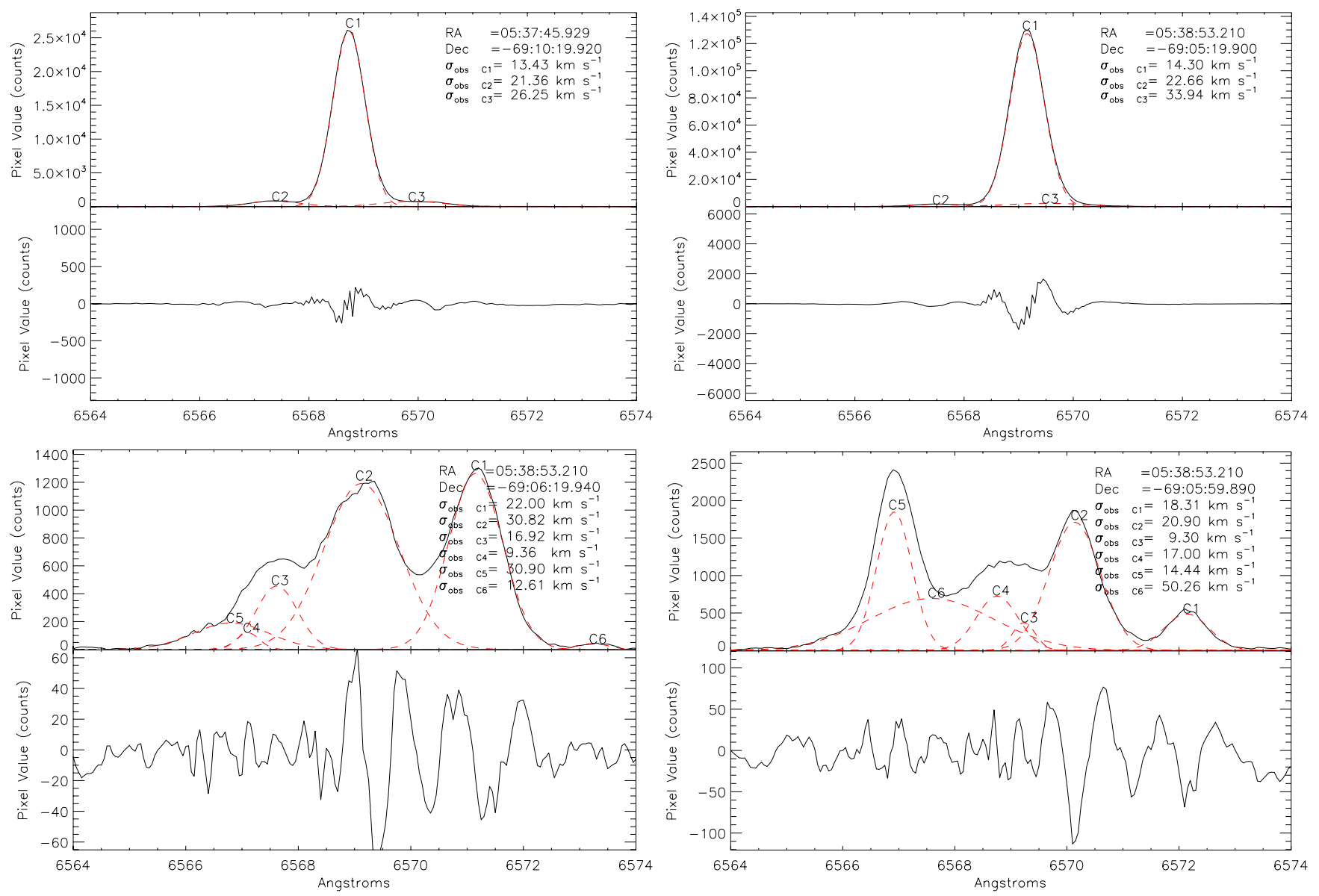

Fig. 8. Multiple Gaussian fits to the $\mathrm{H} \alpha$ profiles in 30 Dor with the narrowest and broadest single-component velocity dispersions (upper and lower panels, respectively). The black (solid) and red (dashed) lines represent the observed and fitted profiles, respectively; each component is numbered. Residuals of the fits are shown below each fit, which are relatively minor when compared with the intensity of the profiles. In each panel we list the position of the spectra and the $\sigma_{\mathrm{obs}}$ of the different fitted components.

than the $\sigma=44 \mathrm{~km} \mathrm{~s}^{-1}$ from Melnick et al. By comparing the bottom panel of Fig. 9 with the Fig. 4 from Melnick et al., we note that our broad component is less intense (with respect to the height of the profile) than their broad fit, perhaps due to the different spatial extents that the two studies cover.

\subsection{The velocity map of 30 Doradus}

As discussed in Sect. 3, we have fitted a single-component Gaussian to each $\mathrm{H} \alpha$ profile. We have used the center and $\sigma$ (corrected by $\sigma_{\text {in }}$ and $\sigma_{\text {th }}$ ) of each fit to derive the radial lineof-sight systemic velocity field and the velocity dispersion map of 30 Dor, as shown in Fig. 10. In the case of the radial velocity field, it was corrected by the systemic velocity of 30 Dor. Although a single Gaussian fit does not represent the exact radial velocity of regions that display multiple profiles, these fits still give us important information regarding the complexity of the observed profiles. i.e., regions that present multiple profiles will have large values of $\sigma$ (as shown in Fig. 7). In this sense, both maps help us to understand the overall dynamics of 30 Dor.

In the top panel of Fig. 10 we show the velocity field, centered on its integrated $\mathrm{H} \alpha$ line (see Fig. 9). Approaching and receding regions are represented by blue and red colors, respectively. Green regions are at the systemic velocity of the gas in 30 Dor. In order to show the whole kinematic behaviour of 30 Doradus, we chose a dynamic range of $70 \mathrm{~km} \mathrm{~s}^{-1}$ in the velocity map. As shown in the velocity map, the north-eastern and south-western regions remain at roughly the systemic velocity $\left(267.4 \mathrm{~km} \mathrm{~s}^{-1}\right.$, see Sect. 4.1). In the case of the south-western region, the velocity dispersion map (lower panel of Fig. 10) shows several narrow $\mathrm{H} \alpha$ profiles, especially in the neighbourhood of 30 Dor B (in which we find the narrowest $\mathrm{H} \alpha$ profile detected in the FLAMES spectra).

Another remarkable feature to note from Fig. 10 is the presence of large expanding structures. The north-eastern edge of shell \#2 (see Chu \& Kennicutt 1994) appears to have negative velocities (with respect to the systemic velocity) while the cavity of this structure appears to have positive velocities, giving a $3 \mathrm{D}$ view of this shell. Several profiles within this cavity display large values for $\sigma_{\mathrm{obs}}$, which are the result of fitting a single Gaussian over at least two $\mathrm{H} \alpha$ components. In general, Fig. 10 shows that the outer regions of 30 Dor are at the same velocity of the integrated profile of all the spectra, and also that the outer regions can typically be fitted by a single Gaussian component.

\subsection{Large expanding structures in 30 Doradus}

We have used the $\mathrm{H} \alpha$ datacube to search for large expanding structures. These structures are characterized by double components in their emission-line profiles, signature of expanding ionized gas at these positions. To identify these structures, we have applied two methods. In a first instance, we have 


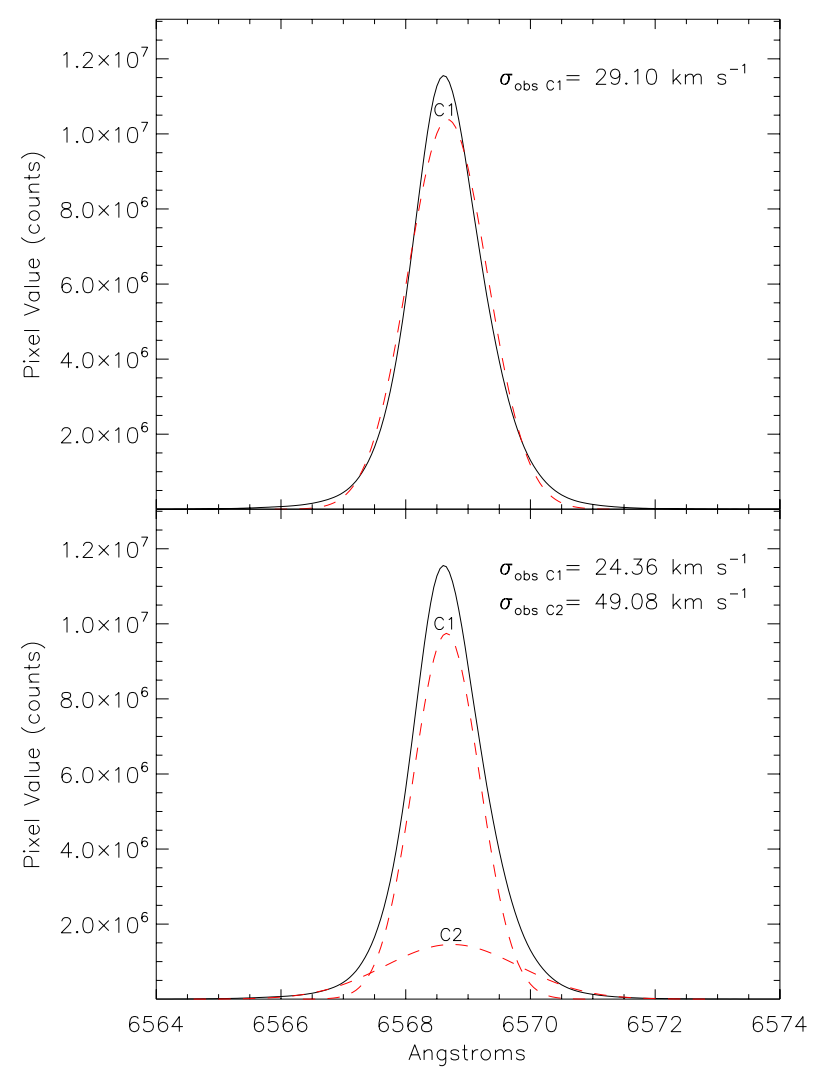

Fig. 9. Single and two-component Gaussian fits to the integrated $\mathrm{H} \alpha$ profile (upper and lower panels, respectively). The fitted components are shown by the red (dashed) lines and their widths are indicated in the upper right of each panel.

integrated the $\mathrm{H} \alpha$ profiles shown in Fig. 3 over regions of $1^{\prime} \times 1^{\prime}$, given the poor spatial sampling of our data. Then, these integrated profiles were visually inspected in order to search for double components. In Fig. 11, we superimpose these integrated $\mathrm{H} \alpha$ profiles over the $\mathrm{H} \alpha$ image. At least ten large expanding structures can be identified and are overlaid on the image in Fig. 11. Some of these - shells 1, 2, 3, and 5 - were catalogued previously (Cox \& Deharveng 1983; Wang \& Helfand 1991), so we adopt the same numbers. Structures 6, 7, 8, 9 and 10 are identified for the first time by this work. These expanding regions present double components in their $\mathrm{H} \alpha$ emission, as can be noted from Fig. 11.

In a second instance, the large expanding structures were searched by using the Gaussian fit on each individual profile. Given that simple $\mathrm{H} \alpha$ profiles can be fitted with a single Gaussian, the $\chi^{2}$ value for these fits will be lower than the value presented by $\mathrm{H} \alpha$ profiles that display double or multiple components. This fact can indicate the location of expanding structures, as profiles that are not well fitted by a single Gaussian. In this sense, we have derived a map with the $\chi^{2}$ values obtained from a single Gaussian fit to the $\mathrm{H} \alpha$ datacube of 30 Dor, which is shown in the bottom panel of Fig. 11. In this case, the Gaussian fit was performed on each observed $\mathrm{H} \alpha$ profile, which was normalized by the total intensity of each profile. Inspecting the bottom panel of Fig. 11, we detect several structures that can not be fitted with a single Gaussian and that could be associated with expanding regions. Interestingly, most of these structures lie on the same position of the expanding regions detected by visual inspection. This correlation is clear for regions \#6 and \#8. Between regions \#1, \#2 and \#8, the $\chi^{2}$ map shows another peak in the
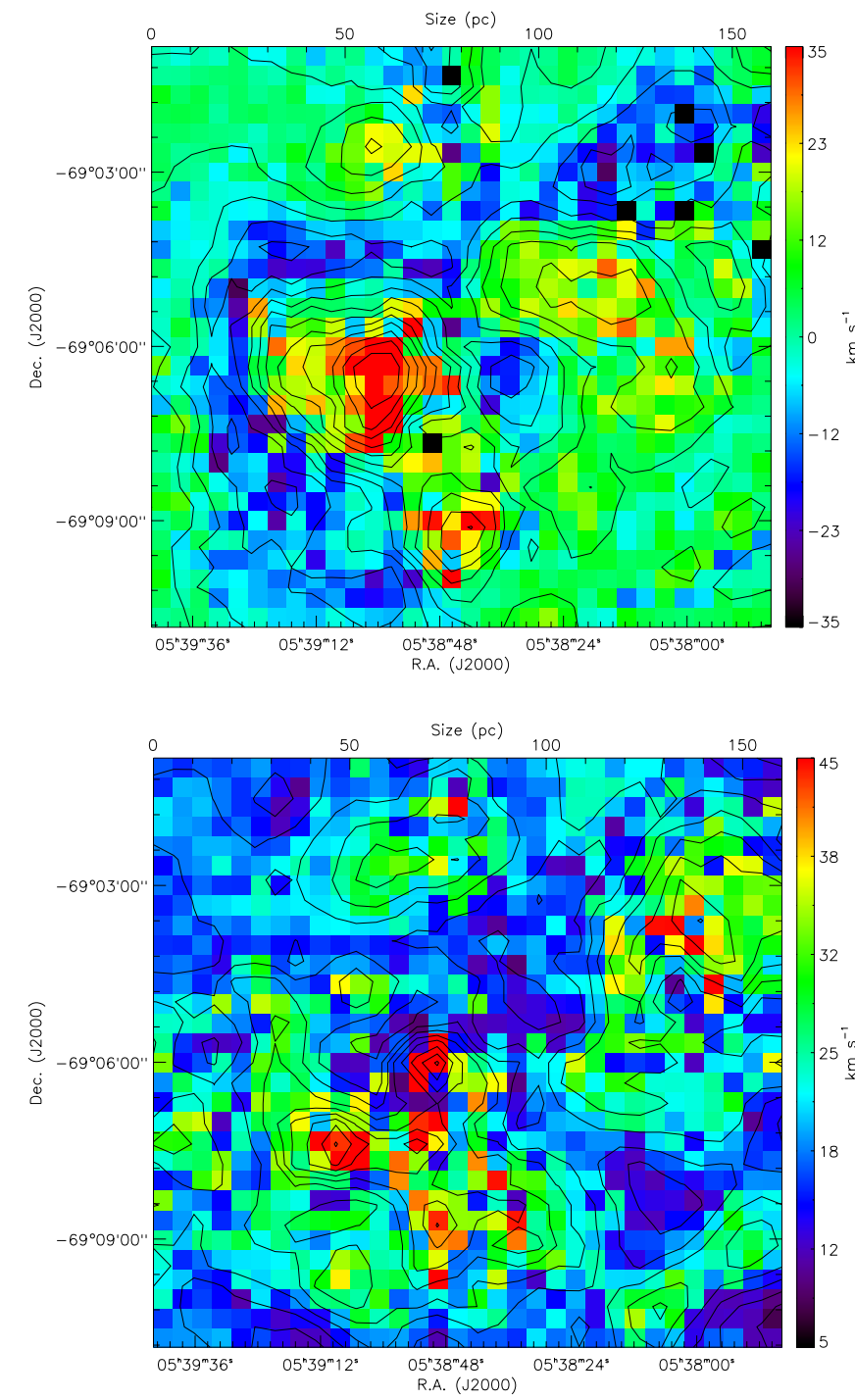

Fig. 10. Radial line-of-sight systemic velocity map (upper panel) and velocity dispersion map (lower panel) of 30 Doradus from analysis of the $\mathrm{H} \alpha$ emission in the FLAMES spectra. North at the top and east to the left. The radial velocity map was corrected by the systemic velocity of 30 Dor.

$\chi^{2}$ values. Inspection of the profiles at that location (top panel of Fig. 11) suggest the presence of a small expanding structure. Despite the analysis of the $\chi^{2}$ map is clearly more quantitative than the visual inspection of the $\mathrm{H} \alpha$ profiles, it results necessary to combine both information in order to determine the size and the real nature of the expanding structures.

Of particular interest is structure \#6. At this position we do not find any star that could be producing an expansion of the ionized gas. Studies of the diffuse X-ray emission seen by the Chandra X-ray Observatory appear to indicate a singletemperature thermal plasma in this region, but with spectral signatures of both collisional ionization equilibrium and nonequilibrium ionization (Townsley et al., in prep.). This may indicate a plasma that has undergone a recent shock and is transitioning back to ionization equilibrium, possibly consistent with a SN remnant. This scenario is compatible with the expanding structure found in this work; we will analyse the main properties of these newly-detected expanding bubbles in a future study.

Another method to search for expanding structures in GHRs is via analysis of long-slit observations, e.g. the analysis in 


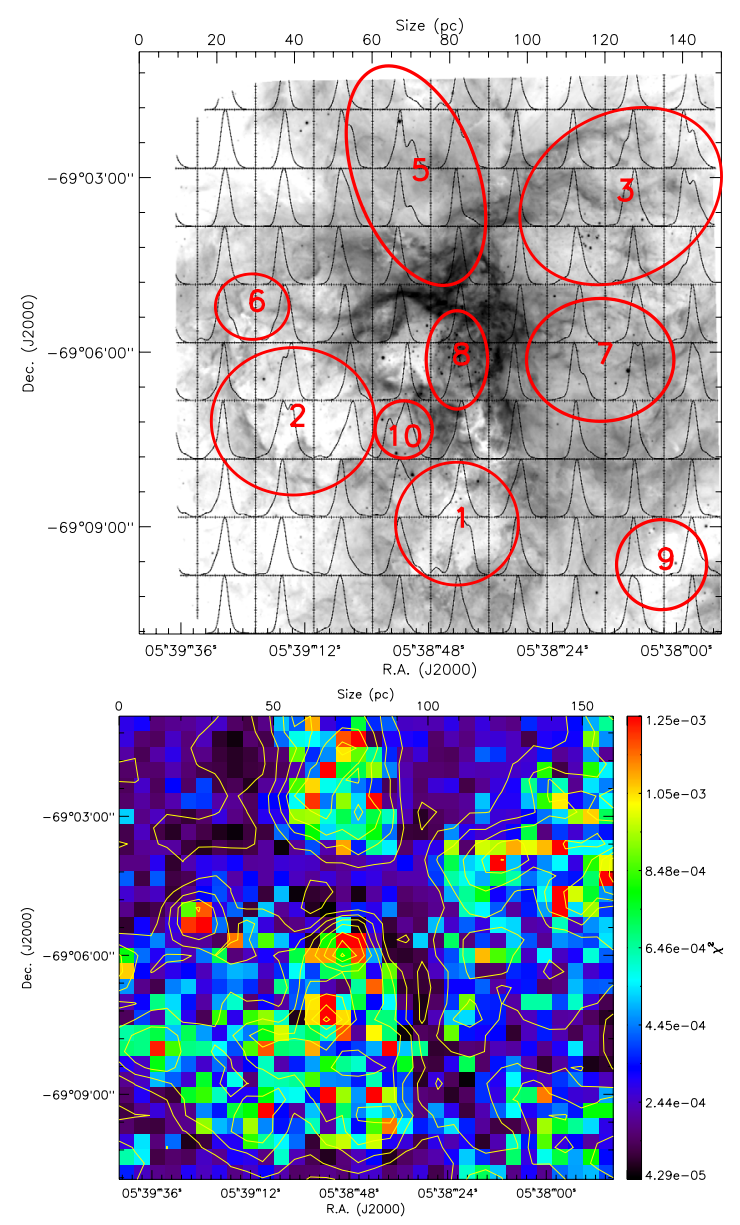

Fig. 11. Top panel: large expanding structures previously catalogued in 30 Dor (nos. 1, 2, 3 and 5) with the five new expanding structures (nos. 6, 7, 8, 9 and 10) identified here. Bottom panel: $\chi^{2}$ map for the $\mathrm{H} \alpha$ cube of 30 Dor, derived from a single Gaussian fit on each observed profile.

30 Dor by Chu \& Kennicutt (1994). To mimic their long-slit observations, we have produced 2D cuts along selected rows of the FLAMES datacube (i.e. at different declinations). In Fig. 12 we show these $2 \mathrm{D}$ cuts of rows $10,11,12,13$ and 14 of the datacube, respectively (see Table A.1 for the RA and Dec of these positions). Row \#10 passes across the southern region of the expanding structure \#2 (see Fig. 11), while rows \#11, \#12, \#13 and \#14 go northwards across the structure \#2. In these spectra, we can clearly see the $\mathrm{H} \alpha$ and [NII] $\lambda \lambda 6548,6584$ lines, with $\mathrm{H} \alpha$ the brightest feature. By inspecting the $\mathrm{H} \alpha$ emission in rows 11-14 from Fig. 12, we can note the presence of an expanding structure (as indicated by the black arrows). This structure, which can be identified with shell \#2 in Fig. 11, appears as a semi-arc of emission close to the center of the $\mathrm{H} \alpha$ line (see the red-dashed ellipse, in the case of row \#14). The expansion of this structure can be clearly identified on the blueward side of the $\mathrm{H} \alpha$ line (see black arrows), but it is not easily seen on the redward side. This structure may be off-center with respect to the systemic $\mathrm{H} \alpha$ emission of 30 Dor.

\section{Summary and conclusions}

We have presented new VLT-FLAMES spectroscopy obtained to study the kinematics of the ionized gas in 30 Doradus. These data consist of regular and irregular grids of nebular
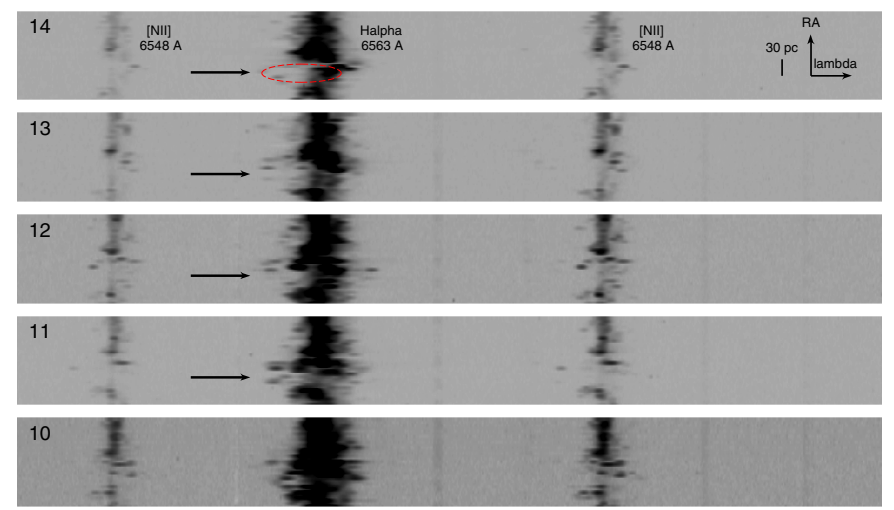

Fig. 12. 2D cut obtained from the spectroscopic datacube of 30 Doradus. These spectra correspond to rows 10, 11, 12, 13 and 14 from bottom to top (the numbers are indicated in the top left corner of each panel). The brightest lines correspond (from left-to-right) to [NII] $\lambda 6548, \mathrm{H} \alpha$ and [NII] $\lambda 6584$. The black arrows indicate the position of an expanding structure. As an example, the red-dashed ellipse indicates the location of this expanding structure in row \#14.

observations, combined with a stellar grid. The regular grid was combined into a datacube, allowing us to analyse the primary kinematic features of 30 Dor, with the main points now briefly summarised.

1. The kinematics of the ionized gas in 30 Dor are complex, with a diverse range of single and multiple $\mathrm{H} \alpha$ profiles. In the brightest regions the $\mathrm{H} \alpha$ profiles are found to be simple and narrow. However, the narrowest $\mathrm{H} \alpha$ profile observed in the data lies close to 30 Dor B, where a past $\mathrm{SN}$ explosion occurred. This is surprising in the sense that 30 Dor B might be expected to be dominated by the kinematics of the SN remnant (i.e., multiple emission profiles and high-velocity components).

2. We have applied multi-Gaussian fits to the $\mathrm{H} \alpha$ profiles of the two narrowest and broadest single-component fits detected in 30 Dor. We do not detect the presence of a broad, lowintensity component, as reported by Melnick et al. (1999) for all of their observed $\mathrm{H} \alpha$ profiles.

3. However, the integrated $\mathrm{H} \alpha$ profile of 30 Dor does display broad wings, and required Gaussian fits that include both narrow and broad components (as per Melnick et al.).

4. We have derived the velocity field and a velocity dispersion map of 30 Dor. By inspecting these maps, we found that the outer parts of our observed regular grid (i.e. a field-of-view of $10^{\prime} \times 10^{\prime}$ centered on R136) are at the same velocity of the integrated $\mathrm{H} \alpha$ profile of 30 Dor.

5. Using the spectroscopic datacube of 30 Dor, we have identified at least five previously unclassified expanding structures. In one case (structure \#6), we did not find any star associated with the expanding gas at that location.

Areas for future work include a detailed analysis of the supersonic velocity in the integrated profile of 30 Dor (besides the origin of the wings in this profile) and a more in-depth study of the individual kinematic structures identified and discussed here.

Acknowledgements. We would like to thank the referee for the useful comments that improved this paper. We also acknowledge the invaluable help of Leisa Townsley in the interpretation of some of the expanding structures in 30 Dor. S.T.-F. acknowledges the financial support of FONDECYT (Chile) through a post-doctoral position, under contract 3110087 . M.R. wishes to acknowledge support from FONDECYT (Chile) grant No. 1080335. M.R. was supported by the Chilean Center for Astrophysics FONDAP No. 15010003. 
S. Torres-Flores et al.: Studying the kinematics of the giant star-forming region 30 Doradus. I.

\section{Appendix A: Position of the fibers}

In Table A.1 we list the fiber positioning of the regular nebular grid (which does not include the positions of the broken fibers). In the cases where we used a fiber from the irregular nebular grid to complete our regular grid, we label that fiber as "IG" (irregular grid). In Table A.2 we list the fiber positions of the irregular nebular grid.

Table A.1. Fiber positions of the regular nebular grid.

\begin{tabular}{|c|c|c|c|c|c|}
\hline ID & $\begin{array}{c}X \\
\text { pix }\end{array}$ & $\begin{array}{c}Y \\
\text { pix }\end{array}$ & $\begin{array}{c}\text { RA (J2000) } \\
\text { h:m:s }\end{array}$ & $\begin{array}{c}\operatorname{Dec}(\mathrm{J} 2000) \\
\mathrm{d}: \mathrm{m}: \mathrm{s}\end{array}$ & Data \\
\hline 1 & 1 & 1 & $05: 39: 41.801$ & $-69: 10: 39.900$ & Obs. \\
\hline 2 & 2 & 1 & $05: 39: 38.059$ & $-69: 10: 39.900$ & Obs. \\
\hline 3 & 3 & 1 & $05: 39: 34.320$ & $-69: 10: 39.900$ & Obs. \\
\hline 4 & 4 & 1 & $05: 39: 30.590$ & $-69: 10: 39.900$ & Obs. \\
\hline 5 & 5 & 1 & $05: 39: 26.849$ & $-69: 10: 39.900$ & Obs. \\
\hline 6 & 6 & 1 & $05: 39: 23.110$ & $-69: 10: 39.900$ & Obs. \\
\hline 7 & 7 & 1 & $05: 39: 19.370$ & $-69: 10: 39.900$ & Obs. \\
\hline 8 & 8 & 1 & $05: 39: 15.629$ & $-69: 10: 39.900$ & Obs. \\
\hline 9 & 9 & 1 & $05: 39: 11.899$ & $-69: 10: 39.900$ & Obs. \\
\hline 10 & 10 & 1 & $05: 39: 08.160$ & $-69: 10: 39.900$ & Obs. \\
\hline 11 & 11 & 1 & $05: 39: 04.421$ & $-69: 10: 39.900$ & Obs. \\
\hline 12 & 12 & 1 & 05:39:00.679 & $-69: 10: 39.900$ & Obs. \\
\hline 13 & 13 & 1 & $05: 38: 56.950$ & $-69: 10: 39.900$ & Obs. \\
\hline 14 & 14 & 1 & $05: 38: 53.210$ & $-69: 10: 39.900$ & Obs. \\
\hline 15 & 15 & 1 & $05: 38: 49.469$ & $-69: 10: 39.900$ & Obs. \\
\hline 16 & 16 & 1 & $05: 38: 45.730$ & $-69: 10: 39.900$ & Obs. \\
\hline 17 & 17 & 1 & $05: 38: 42.000$ & $-69: 10: 39.900$ & Obs. \\
\hline 18 & 18 & 1 & $05: 38: 38.261$ & $-69: 10: 39.900$ & Obs. \\
\hline 19 & 19 & 1 & $05: 38: 34.519$ & $-69: 10: 39.900$ & Obs. \\
\hline 20 & 20 & 1 & $05: 38: 30.780$ & $-69: 10: 39.900$ & Obs. \\
\hline 21 & 21 & 1 & $05: 38: 27.050$ & $-69: 10: 39.900$ & Obs. \\
\hline 22 & 22 & 1 & $05: 38: 23.309$ & $-69: 10: 39.900$ & Obs. \\
\hline 23 & 23 & 1 & $05: 38: 19.570$ & $-69: 10: 39.900$ & Obs. \\
\hline 24 & 24 & 1 & $05: 38: 15.830$ & $-69: 10: 39.900$ & Obs. \\
\hline 25 & 25 & 1 & $05: 38: 12.101$ & $-69: 10: 39.900$ & Obs. \\
\hline 26 & 26 & 1 & 05:38:08.359 & $-69: 10: 39.900$ & Obs. \\
\hline 27 & 27 & 1 & $05: 38: 04.620$ & $-69: 10: 39.900$ & Obs. \\
\hline 28 & 28 & 1 & 05:38:00.881 & $-69: 10: 39.900$ & Obs. \\
\hline 29 & 29 & 1 & $05: 37: 57.139$ & $-69: 10: 39.900$ & Obs. \\
\hline 30 & 30 & 1 & $05: 37: 53.410$ & $-69: 10: 39.900$ & Obs. \\
\hline 31 & 31 & 1 & $05: 37: 49.670$ & $-69: 10: 39.900$ & Obs. \\
\hline 32 & 32 & 1 & $05: 37: 45.929$ & $-69: 10: 39.900$ & Obs. \\
\hline 33 & 1 & 2 & $05: 39: 41.801$ & $-69: 10: 19.920$ & Obs. \\
\hline 34 & 2 & 2 & $05: 39: 38.059$ & $-69: 10: 19.920$ & Obs. \\
\hline 35 & 3 & 2 & $05: 39: 34.320$ & $-69: 10: 19.920$ & Obs. \\
\hline 36 & 4 & 2 & $05: 39: 30.590$ & $-69: 10: 19.920$ & Obs. \\
\hline 37 & 5 & 2 & $05: 39: 26.849$ & $-69: 10: 19.920$ & Obs. \\
\hline 38 & 6 & 2 & $05: 39: 23.110$ & $-69: 10: 19.920$ & Obs. \\
\hline 39 & 7 & 2 & $05: 39: 19.370$ & $-69: 10: 19.920$ & Obs. \\
\hline 40 & 8 & 2 & $05: 39: 15.629$ & $-69: 10: 19.920$ & Obs. \\
\hline 41 & 9 & 2 & 05:39:11.899 & $-69: 10: 19.920$ & Obs. \\
\hline 42 & 10 & 2 & $05: 39: 08.160$ & $-69: 10: 19.920$ & Obs. \\
\hline 43 & 11 & 2 & 05:39:04.421 & $-69: 10: 19.920$ & Obs. \\
\hline 44 & 12 & 2 & 05:39:00.679 & $-69: 10: 19.920$ & Obs. \\
\hline 45 & 13 & 2 & $05: 38: 56.950$ & $-69: 10: 19.920$ & Obs. \\
\hline 46 & 14 & 2 & $05: 38: 53.210$ & $-69: 10: 19.920$ & Obs. \\
\hline 47 & 15 & 2 & $05: 38: 49.469$ & $-69: 10: 19.920$ & Obs. \\
\hline 48 & 16 & 2 & $05: 38: 45.730$ & $-69: 10: 19.920$ & Obs. \\
\hline 49 & 17 & 2 & $05: 38: 42.000$ & $-69: 10: 19.920$ & Obs. \\
\hline 50 & 18 & 2 & $05: 38: 38.261$ & $-69: 10: 19.920$ & Obs. \\
\hline 51 & 19 & 2 & $05: 38: 34.519$ & $-69: 10: 19.920$ & Obs. \\
\hline 52 & 20 & 2 & $05: 38: 30.780$ & $-69: 10: 19.920$ & Obs. \\
\hline 53 & 21 & 2 & $05: 38: 27.050$ & $-69: 10: 19.920$ & Obs. \\
\hline 54 & 22 & 2 & $05: 38: 23.309$ & $-69: 10: 19.920$ & Obs. \\
\hline 55 & 23 & 2 & $05: 38: 19.570$ & $-69: 10: 19.920$ & Obs. \\
\hline 56 & 24 & 2 & $05: 38: 15.830$ & $-69: 10: 19.920$ & Obs. \\
\hline
\end{tabular}

Table A.1. continued

\begin{tabular}{|c|c|c|c|c|c|}
\hline ID & $\begin{array}{c}X \\
\text { pix }\end{array}$ & $\begin{array}{c}Y \\
\text { pix }\end{array}$ & $\begin{array}{c}\text { RA (J2000) } \\
\text { h:m:s }\end{array}$ & $\begin{array}{c}\operatorname{Dec}(\mathrm{J} 2000) \\
\mathrm{d}: \mathrm{m}: \mathrm{s}\end{array}$ & Data \\
\hline 57 & 25 & 2 & $05: 38: 12.101$ & $-69: 10: 19.920$ & Obs. \\
\hline 58 & 26 & 2 & 05:38:08.359 & $-69: 10: 19.920$ & Obs. \\
\hline 59 & 27 & 2 & 05:38:04.620 & $-69: 10: 19.920$ & Obs. \\
\hline 60 & 28 & 2 & 05:38:00.881 & $-69: 10: 19.920$ & Obs. \\
\hline 61 & 29 & 2 & $05: 37: 57.139$ & $-69: 10: 19.920$ & Obs. \\
\hline 62 & 30 & 2 & $05: 37: 53.410$ & $-69: 10: 19.920$ & Obs. \\
\hline 63 & 31 & 2 & $05: 37: 49.670$ & $-69: 10: 19.920$ & Obs. \\
\hline 64 & 32 & 2 & $05: 37: 45.929$ & $-69: 10: 19.920$ & Obs. \\
\hline 65 & 1 & 3 & $05: 39: 41.796$ & $-69: 09: 59.900$ & Obs. \\
\hline 66 & 2 & 3 & $05: 39: 38.059$ & $-69: 09: 59.900$ & Obs. \\
\hline 67 & 3 & 3 & $05: 39: 34.325$ & $-69: 09: 59.900$ & Obs. \\
\hline 68 & 4 & 3 & $05: 39: 30.590$ & $-69: 09: 59.900$ & Obs. \\
\hline 69 & 5 & 3 & $05: 39: 26.846$ & $-69: 09: 59.900$ & Obs. \\
\hline 70 & 6 & 3 & $05: 39: 23.110$ & $-69: 09: 59.900$ & Obs. \\
\hline 71 & 7 & 3 & $05: 39: 19.366$ & $-69: 09: 59.900$ & Obs. \\
\hline 72 & 8 & 3 & $05: 39: 15.629$ & $-69: 09: 59.900$ & Obs. \\
\hline 73 & 9 & 3 & 05:39:11.894 & $-69: 09: 59.900$ & Obs. \\
\hline 74 & 10 & 3 & 05:39:08.160 & $-69: 09: 59.900$ & Obs. \\
\hline 75 & 11 & 3 & $05: 39: 04.416$ & $-69: 09: 59.900$ & Obs. \\
\hline 76 & 12 & 3 & 05:39:00.679 & $-69: 09: 59.900$ & Obs. \\
\hline 77 & 13 & 3 & $05: 38: 56.945$ & $-69: 09: 59.900$ & Obs. \\
\hline 78 & 14 & 3 & $05: 38: 53.210$ & $-69: 09: 59.900$ & Obs. \\
\hline 79 & 15 & 3 & $05: 38: 49.466$ & $-69: 09: 59.900$ & Obs. \\
\hline 80 & 16 & 3 & $05: 38: 45.730$ & $-69: 09: 59.900$ & Obs. \\
\hline 81 & 17 & 3 & 05:38:41.995 & $-69: 09: 59.900$ & Obs. \\
\hline 82 & 18 & 3 & $05: 38: 38.261$ & $-69: 09: 59.900$ & Obs. \\
\hline 83 & 19 & 3 & $05: 38: 34.514$ & $-69: 09: 59.900$ & Obs. \\
\hline 84 & 20 & 3 & $05: 38: 30.780$ & $-69: 09: 59.900$ & Obs. \\
\hline 85 & 21 & 3 & $05: 38: 27.050$ & $-69: 09: 59.900$ & Obs. \\
\hline 86 & 22 & 3 & 05:38:23.309 & $-69: 09: 59.900$ & Obs. \\
\hline 87 & 23 & 3 & $05: 38: 19.565$ & $-69: 09: 59.900$ & Obs. \\
\hline 88 & 24 & 3 & $05: 38: 15.830$ & $-69: 09: 59.900$ & Obs. \\
\hline 89 & 25 & 3 & $05: 38: 12.096$ & $-69: 09: 59.900$ & Obs. \\
\hline 90 & 26 & 3 & 05:38:08.359 & $-69: 09: 59.900$ & Obs. \\
\hline 91 & 27 & 3 & 05:38:04.615 & $-69: 09: 59.900$ & Obs. \\
\hline 92 & 28 & 3 & 05:38:00.881 & $-69: 09: 59.900$ & Obs. \\
\hline 93 & 29 & 3 & $05: 37: 57.146$ & $-69: 09: 59.900$ & Obs. \\
\hline 94 & 30 & 3 & $05: 37: 53.410$ & $-69: 09: 59.900$ & Obs. \\
\hline 95 & 31 & 3 & $05: 37: 49.666$ & $-69: 09: 59.900$ & Obs. \\
\hline 96 & 32 & 3 & $05: 37: 45.929$ & $-69: 09: 59.900$ & Obs. \\
\hline 97 & 1 & 4 & $05: 39: 41.796$ & $-69: 09: 39.850$ & Obs. \\
\hline 98 & 2 & 4 & $05: 39: 38.059$ & $-69: 09: 39.920$ & Obs. \\
\hline 99 & 3 & 4 & $05: 39: 34.325$ & $-69: 09: 39.920$ & Obs. \\
\hline 100 & 4 & 4 & $05: 39: 30.590$ & $-69: 09: 39.920$ & Obs. \\
\hline 101 & 5 & 4 & $05: 39: 26.846$ & $-69: 09: 39.920$ & Obs. \\
\hline 102 & 6 & 4 & $05: 39: 23.110$ & $-69: 09: 39.920$ & Obs. \\
\hline 103 & 7 & 4 & 05:39:19.366 & $-69: 09: 39.850$ & Obs. \\
\hline 104 & 8 & 4 & $05: 39: 15.629$ & $-69: 09: 39.920$ & Obs. \\
\hline 105 & 9 & 4 & 05:39:11.894 & $-69: 09: 39.850$ & Obs. \\
\hline 106 & 10 & 4 & 05:39:08.160 & $-69: 09: 39.920$ & Obs. \\
\hline 107 & 11 & 4 & 05:39:04.416 & $-69: 09: 39.920$ & Obs. \\
\hline 108 & 12 & 4 & 05:39:00.679 & $-69: 09: 39.920$ & Obs. \\
\hline 109 & 13 & 4 & $05: 38: 56.945$ & $-69: 09: 39.920$ & Obs. \\
\hline 110 & 14 & 4 & $05: 38: 53.210$ & $-69: 09: 39.920$ & Obs. \\
\hline 111 & 15 & 4 & $05: 38: 49.466$ & $-69: 09: 39.920$ & Obs. \\
\hline 112 & 16 & 4 & $05: 38: 45.730$ & $-69: 09: 39.920$ & Obs. \\
\hline 113 & 17 & 4 & 05:38:41.995 & $-69: 09: 39.920$ & Obs. \\
\hline 114 & 18 & 4 & $05: 38: 38.261$ & $-69: 09: 39.920$ & Obs. \\
\hline 115 & 19 & 4 & $05: 38: 34.514$ & $-69: 09: 39.920$ & Obs. \\
\hline 116 & 20 & 4 & $05: 38: 30.780$ & $-69: 09: 39.920$ & Obs. \\
\hline 117 & 21 & 4 & $05: 38: 27.050$ & $-69: 09: 39.920$ & Obs. \\
\hline 118 & 22 & 4 & 05:38:23.309 & $-69: 09: 39.920$ & Obs. \\
\hline 119 & 23 & 4 & $05: 38: 19.565$ & $-69: 09: 39.920$ & Obs. \\
\hline 120 & 24 & 4 & $05: 38: 15.830$ & $-69: 09: 39.920$ & Obs. \\
\hline 121 & 25 & 4 & 05:38:12.096 & $-69: 09: 39.920$ & Obs. \\
\hline
\end{tabular}


Table A.1. continued.

\begin{tabular}{|c|c|c|c|c|c|}
\hline ID & $\begin{array}{c}X \\
\text { pix }\end{array}$ & $\begin{array}{c}Y \\
\text { pix }\end{array}$ & $\begin{array}{c}\text { RA (J2000) } \\
\text { h:m:s }\end{array}$ & $\begin{array}{c}\text { Dec (J2000) } \\
\text { d:m:s }\end{array}$ & Data \\
\hline 122 & 26 & 4 & $05: 38: 08.359$ & $-69: 09: 39.920$ & Obs. \\
\hline 123 & 27 & 4 & 05:38:04.615 & $-69: 09: 39.920$ & Obs. \\
\hline 124 & 28 & 4 & 05:38:00.881 & $-69: 09: 39.920$ & Obs. \\
\hline 125 & 29 & 4 & $05: 37: 57.146$ & $-69: 09: 39.920$ & Obs. \\
\hline 126 & 30 & 4 & $05: 37: 53.410$ & $-69: 09: 39.920$ & Obs. \\
\hline 127 & 31 & 4 & $05: 37: 49.666$ & $-69: 09: 39.920$ & Obs. \\
\hline 128 & 32 & 4 & $05: 37: 45.929$ & $-69: 09: 39.920$ & Obs. \\
\hline 129 & 1 & 5 & 05:39:41.796 & $-69: 09: 19.910$ & Obs. \\
\hline 130 & 2 & 5 & 05:39:38.059 & $-69: 09: 19.910$ & Obs. \\
\hline 131 & 3 & 5 & 05:39:34.325 & $-69: 09: 19.910$ & Obs. \\
\hline 132 & 4 & 5 & 05:39:30.590 & $-69: 09: 19.910$ & Obs. \\
\hline 133 & 5 & 5 & $05: 39: 26.846$ & $-69: 09: 19.910$ & Obs. \\
\hline 134 & 6 & 5 & 05:39:23.110 & $-69: 09: 19.910$ & Obs. \\
\hline 135 & 7 & 5 & 05:39:19.366 & $-69: 09: 19.910$ & Obs. \\
\hline 136 & 8 & 5 & 05:39:15.629 & $-69: 09: 19.910$ & Obs. \\
\hline 137 & 9 & 5 & 05:39:11.894 & $-69: 09: 19.910$ & Obs. \\
\hline 138 & 10 & 5 & $05: 39: 08.160$ & $-69: 09: 19.910$ & Obs. \\
\hline 139 & 11 & 5 & 05:39:04.416 & $-69: 09: 19.910$ & Obs. \\
\hline 140 & 12 & 5 & 05:39:00.679 & $-69: 09: 19.910$ & Obs. \\
\hline 141 & 13 & 5 & 05:38:56.945 & $-69: 09: 19.910$ & Obs. \\
\hline 142 & 14 & 5 & $05: 38: 53.210$ & $-69: 09: 19.910$ & Obs. \\
\hline 143 & 15 & 5 & 05:38:49.466 & $-69: 09: 19.910$ & Obs. \\
\hline 144 & 16 & 5 & $05: 38: 45.730$ & $-69: 09: 19.910$ & Obs. \\
\hline 145 & 17 & 5 & 05:38:41.995 & $-69: 09: 19.910$ & Obs. \\
\hline 146 & 18 & 5 & 05:38:38.261 & $-69: 09: 19.910$ & Obs. \\
\hline 147 & 19 & 5 & $05: 38: 34.514$ & $-69: 09: 19.910$ & Obs. \\
\hline 148 & 20 & 5 & 05:38:30.780 & $-69: 09: 19.910$ & Obs. \\
\hline 149 & 21 & 5 & $05: 38: 27.050$ & $-69: 09: 19.910$ & Obs. \\
\hline 150 & 22 & 5 & 05:38:23.309 & $-69: 09: 19.910$ & Obs. \\
\hline 151 & 23 & 5 & $05: 38: 19.565$ & $-69: 09: 19.910$ & Obs. \\
\hline 152 & 24 & 5 & $05: 38: 15.830$ & $-69: 09: 19.910$ & Obs. \\
\hline 153 & 25 & 5 & 05:38:12.096 & $-69: 09: 19.910$ & Obs. \\
\hline 154 & 26 & 5 & 05:38:08.359 & $-69: 09: 19.910$ & Obs. \\
\hline 155 & 27 & 5 & 05:38:04.615 & $-69: 09: 19.910$ & Obs. \\
\hline 156 & 28 & 5 & 05:38:00.881 & $-69: 09: 19.910$ & Obs. \\
\hline 157 & 29 & 5 & 05:37:57.146 & $-69: 09: 19.910$ & Obs. \\
\hline 158 & 30 & 5 & $05: 37: 53.410$ & $-69: 09: 19.910$ & Obs. \\
\hline 159 & 31 & 5 & 05:37:49.666 & $-69: 09: 19.910$ & Obs. \\
\hline 160 & 32 & 5 & $05: 37: 45.929$ & $-69: 09: 19.910$ & Obs. \\
\hline 161 & 1 & 6 & 05:39:41.796 & $-69: 08: 59.860$ & Obs. \\
\hline 162 & 2 & 6 & 05:39:38.059 & $-69: 08: 59.930$ & Obs. \\
\hline 163 & 3 & 6 & 05:39:34.325 & $-69: 08: 59.930$ & Obs. \\
\hline 164 & 4 & 6 & 05:39:30.590 & $-69: 08: 59.930$ & Obs. \\
\hline 165 & 5 & 6 & 05:39:26.846 & $-69: 08: 59.930$ & Obs. \\
\hline 166 & 6 & 6 & 05:39:23.110 & $-69: 08: 59.930$ & Obs. \\
\hline 167 & 7 & 6 & 05:39:19.366 & $-69: 08: 59.860$ & Obs. \\
\hline 168 & 8 & 6 & 05:39:15.629 & $-69: 08: 59.930$ & Obs. \\
\hline 169 & 9 & 6 & 05:39:11.894 & $-69: 08: 59.860$ & Obs. \\
\hline 170 & 10 & 6 & 05:39:08.160 & $-69: 08: 59.930$ & Obs. \\
\hline 171 & 11 & 6 & 05:39:04.416 & $-69: 08: 59.930$ & Obs. \\
\hline 172 & 12 & 6 & 05:39:00.679 & $-69: 08: 59.930$ & Obs. \\
\hline 173 & 13 & 6 & 05:38:56.945 & $-69: 08: 59.930$ & Obs. \\
\hline 174 & 14 & 6 & 05:38:53.210 & $-69: 08: 59.930$ & Obs. \\
\hline 175 & 15 & 6 & $05: 38: 49.466$ & $-69: 08: 59.930$ & Obs. \\
\hline 176 & 16 & 6 & $05: 38: 45.730$ & $-69: 08: 59.930$ & Obs. \\
\hline 177 & 17 & 6 & 05:38:41.995 & $-69: 08: 59.930$ & Obs. \\
\hline 178 & 18 & 6 & $05: 38: 38.261$ & $-69: 08: 59.930$ & Obs. \\
\hline 179 & 19 & 6 & 05:38:34.514 & $-69: 08: 59.930$ & Obs. \\
\hline 180 & 20 & 6 & $05: 38: 30.780$ & $-69: 08: 59.930$ & Obs. \\
\hline 181 & 21 & 6 & $05: 38: 27.050$ & $-69: 08: 59.930$ & Obs. \\
\hline 182 & 22 & 6 & 05:38:23.309 & $-69: 08: 59.930$ & Obs. \\
\hline 183 & 23 & 6 & 05:38:19.565 & $-69: 08: 59.930$ & Obs. \\
\hline 184 & 24 & 6 & $05: 38: 15.830$ & $-69: 08: 59.930$ & Obs. \\
\hline 185 & 25 & 6 & 05:38:12.096 & $-69: 08: 59.930$ & Obs. \\
\hline
\end{tabular}

Table A.1. continued.

\begin{tabular}{|c|c|c|c|c|c|}
\hline ID & $\begin{array}{c}X \\
\text { pix }\end{array}$ & $\begin{array}{c}Y \\
\text { pix }\end{array}$ & $\begin{array}{c}\text { RA (J2000) } \\
\text { h:m:s }\end{array}$ & $\begin{array}{c}\operatorname{Dec}(\mathrm{J} 2000) \\
\text { d:m:s }\end{array}$ & Data \\
\hline 186 & 26 & 6 & $05: 38: 08.359$ & $-69: 08: 59.930$ & Obs. \\
\hline 187 & 27 & 6 & 05:38:04.615 & $-69: 08: 59.930$ & Obs. \\
\hline 188 & 28 & 6 & 05:38:00.881 & $-69: 08: 59.930$ & Obs. \\
\hline 189 & 29 & 6 & $05: 37: 57.146$ & $-69: 08: 59.930$ & Obs. \\
\hline 190 & 30 & 6 & $05: 37: 53.410$ & $-69: 08: 59.930$ & Obs. \\
\hline 191 & 31 & 6 & $05: 37: 49.666$ & $-69: 08: 59.930$ & Obs. \\
\hline 192 & 32 & 6 & $05: 37: 45.929$ & $-69: 08: 59.930$ & Obs. \\
\hline 193 & 1 & 7 & $05: 39: 41.796$ & $-69: 08: 39.910$ & Obs. \\
\hline 194 & 2 & 7 & 05:39:38.059 & $-69: 08: 39.910$ & Obs. \\
\hline 195 & 3 & 7 & $05: 39: 34.325$ & $-69: 08: 39.910$ & Obs. \\
\hline 196 & 4 & 7 & $05: 39: 30.590$ & $-69: 08: 39.910$ & Obs. \\
\hline 197 & 5 & 7 & $05: 39: 26.846$ & $-69: 08: 39.910$ & Obs. \\
\hline 198 & 6 & 7 & $05: 39: 23.110$ & $-69: 08: 39.910$ & Obs. \\
\hline 199 & 7 & 7 & $05: 39: 19.366$ & $-69: 08: 39.910$ & Obs. \\
\hline 200 & 8 & 7 & $05: 39: 15.629$ & $-69: 08: 39.910$ & Obs. \\
\hline 201 & 9 & 7 & 05:39:11.894 & $-69: 08: 39.910$ & Obs. \\
\hline 202 & 10 & 7 & 05:39:08.160 & $-69: 08: 39.910$ & Obs. \\
\hline 203 & 11 & 7 & 05:39:04.416 & $-69: 08: 39.910$ & Obs. \\
\hline 204 & 12 & 7 & 05:39:00.679 & $-69: 08: 39.910$ & Obs. \\
\hline 205 & 13 & 7 & $05: 38: 56.945$ & $-69: 08: 39.910$ & Obs. \\
\hline 206 & 14 & 7 & $05: 38: 53.210$ & $-69: 08: 39.910$ & Obs. \\
\hline 207 & 15 & 7 & $05: 38: 49.466$ & $-69: 08: 39.910$ & Obs. \\
\hline 208 & 16 & 7 & $05: 38: 45.730$ & $-69: 08: 39.910$ & Obs. \\
\hline 209 & 17 & 7 & 05:38:41.995 & $-69: 08: 39.910$ & Obs. \\
\hline 210 & 18 & 7 & $05: 38: 38.261$ & $-69: 08: 39.910$ & Obs. \\
\hline 211 & 19 & 7 & $05: 38: 34.514$ & $-69: 08: 39.910$ & Obs. \\
\hline 212 & 20 & 7 & 05:38:30.780 & $-69: 08: 39.910$ & Obs. \\
\hline 213 & 21 & 7 & $05: 38: 27.046$ & $-69: 08: 39.910$ & Obs. \\
\hline 214 & 22 & 7 & 05:38:23.309 & $-69: 08: 39.910$ & Obs. \\
\hline 215 & 23 & 7 & $05: 38: 19.565$ & $-69: 08: 39.910$ & Obs. \\
\hline 216 & 24 & 7 & $05: 38: 15.830$ & $-69: 08: 39.910$ & Obs. \\
\hline 217 & 25 & 7 & $05: 38: 12.096$ & $-69: 08: 39.910$ & Obs. \\
\hline 218 & 26 & 7 & 05:38:08.359 & $-69: 08: 39.910$ & Obs. \\
\hline 219 & 27 & 7 & 05:38:04.615 & $-69: 08: 39.910$ & Obs. \\
\hline 220 & 28 & 7 & 05:38:00.881 & $-69: 08: 39.910$ & Obs. \\
\hline 221 & 29 & 7 & $05: 37: 57.146$ & $-69: 08: 39.910$ & Obs. \\
\hline 222 & 30 & 7 & $05: 37: 53.410$ & $-69: 08: 39.910$ & Obs. \\
\hline 223 & 31 & 7 & $05: 37: 49.666$ & $-69: 08: 39.910$ & Obs. \\
\hline 224 & 32 & 7 & $05: 37: 45.929$ & $-69: 08: 39.910$ & Obs. \\
\hline 225 & 1 & 8 & 05:39:41.796 & $-69: 08: 19.860$ & Obs. \\
\hline 226 & 2 & 8 & $05: 39: 38.059$ & $-69: 08: 19.930$ & Obs. \\
\hline 227 & 3 & 8 & $05: 39: 34.325$ & $-69: 08: 19.930$ & Obs. \\
\hline 228 & 4 & 8 & $05: 39: 30.590$ & $-69: 08: 19.930$ & Obs. \\
\hline 229 & 5 & 8 & $05: 39: 26.846$ & $-69: 08: 19.930$ & Obs. \\
\hline 230 & 6 & 8 & $05: 39: 23.110$ & $-69: 08: 19.930$ & Obs. \\
\hline 231 & 7 & 8 & $05: 39: 19.366$ & $-69: 08: 19.860$ & Obs. \\
\hline 232 & 8 & 8 & $05: 39: 15.629$ & $-69: 08: 19.930$ & Obs. \\
\hline 233 & 9 & 8 & 05:39:11.894 & $-69: 08: 19.860$ & Obs. \\
\hline 234 & 10 & 8 & 05:39:08.160 & $-69: 08: 19.930$ & Obs. \\
\hline 235 & 11 & 8 & 05:39:04.416 & $-69: 08: 19.930$ & Obs. \\
\hline 236 & 12 & 8 & 05:39:00.679 & $-69: 08: 19.930$ & Obs. \\
\hline 237 & 13 & 8 & $05: 38: 56.945$ & $-69: 08: 19.930$ & Obs. \\
\hline 238 & 14 & 8 & 05:38:53.210 & $-69: 08: 19.930$ & Obs. \\
\hline 239 & 15 & 8 & $05: 38: 49.466$ & $-69: 08: 19.860$ & Obs. \\
\hline 240 & 16 & 8 & $05: 38: 45.730$ & $-69: 08: 19.930$ & Obs. \\
\hline 241 & 17 & 8 & 05:38:41.995 & $-69: 08: 19.860$ & Obs. \\
\hline 242 & 18 & 8 & $05: 38: 38.261$ & $-69: 08: 19.930$ & Obs. \\
\hline 243 & 19 & 8 & $05: 38: 34.514$ & $-69: 08: 19.860$ & Obs. \\
\hline 244 & 20 & 8 & $05: 38: 30.780$ & $-69: 08: 19.930$ & Obs. \\
\hline 245 & 21 & 8 & $05: 38: 27.046$ & $-69: 08: 19.860$ & Obs. \\
\hline 246 & 22 & 8 & 05:38:23.309 & $-69: 08: 19.930$ & Obs. \\
\hline 247 & 23 & 8 & $05: 38: 19.565$ & $-69: 08: 19.860$ & Obs. \\
\hline 248 & 24 & 8 & $05: 38: 15.830$ & $-69: 08: 19.930$ & Obs. \\
\hline 249 & 25 & 8 & $05: 38: 12.096$ & $-69: 08: 19.860$ & Obs. \\
\hline
\end{tabular}


S. Torres-Flores et al.: Studying the kinematics of the giant star-forming region 30 Doradus. I.

Table A.1. continued.

\begin{tabular}{|c|c|c|c|c|c|}
\hline ID & $\begin{array}{c}X \\
\text { pix }\end{array}$ & $\begin{array}{c}Y \\
\text { pix }\end{array}$ & $\begin{array}{l}\text { RA (J2000) } \\
\text { h:m:s }\end{array}$ & $\begin{array}{c}\text { Dec (J2000) } \\
\text { d:m:s }\end{array}$ & Data \\
\hline 250 & 26 & 8 & $05: 38: 08.359$ & $-69: 08: 19.930$ & Obs. \\
\hline 251 & 27 & 8 & 05:38:04.615 & $-69: 08: 19.860$ & Obs. \\
\hline 252 & 28 & 8 & 05:38:00.881 & $-69: 08: 19.930$ & Obs. \\
\hline 253 & 29 & 8 & 05:37:57.146 & $-69: 08: 19.860$ & Obs. \\
\hline 254 & 30 & 8 & $05: 37: 53.410$ & $-69: 08: 19.930$ & Obs. \\
\hline 255 & 31 & 8 & 05:37:49.666 & $-69: 08: 19.860$ & Obs. \\
\hline 256 & 32 & 8 & 05:37:45.929 & $-69: 08: 19.930$ & Obs. \\
\hline 257 & 1 & 9 & 05:39:41.796 & $-69: 07: 59.920$ & Obs. \\
\hline 258 & 2 & 9 & 05:39:38.059 & $-69: 07: 59.920$ & Obs. \\
\hline 259 & 3 & 9 & $05: 39: 34.325$ & $-69: 07: 59.920$ & Obs. \\
\hline 260 & 4 & 9 & 05:39:30.590 & $-69: 07: 59.920$ & Obs. \\
\hline 261 & 5 & 9 & $05: 39: 26.846$ & $-69: 07: 59.920$ & Obs. \\
\hline 262 & 6 & 9 & 05:39:23.110 & $-69: 07: 59.920$ & Obs. \\
\hline 263 & 7 & 9 & 05:39:19.366 & $-69: 07: 59.920$ & Obs. \\
\hline 264 & 8 & 9 & 05:39:15.629 & $-69: 07: 59.920$ & Obs. \\
\hline 265 & 9 & 9 & 05:39:11.894 & $-69: 07: 59.920$ & Obs. \\
\hline 266 & 10 & 9 & 05:39:08.160 & $-69: 07: 59.920$ & Obs. \\
\hline 267 & 11 & 9 & 05:39:04.416 & $-69: 07: 59.920$ & Obs. \\
\hline 268 & 12 & 9 & 05:39:00.679 & $-69: 07: 59.920$ & Obs. \\
\hline 269 & 13 & 9 & 05:38:56.945 & $-69: 07: 59.920$ & Obs. \\
\hline 270 & 14 & 9 & 05:38:53.210 & $-69: 07: 59.920$ & Obs. \\
\hline 271 & 15 & 9 & 05:38:49.466 & $-69: 07: 59.920$ & Obs. \\
\hline 272 & 16 & 9 & $05: 38: 45.730$ & $-69: 07: 59.920$ & Obs. \\
\hline 273 & 17 & 9 & 05:38:41.995 & $-69: 07: 59.920$ & Obs. \\
\hline 274 & 18 & 9 & 05:38:38.261 & $-69: 07: 59.920$ & Obs. \\
\hline 275 & 19 & 9 & $05: 38: 34.514$ & $-69: 07: 59.920$ & Obs. \\
\hline 276 & 20 & 9 & 05:38:30.780 & $-69: 07: 59.920$ & Obs. \\
\hline 277 & 21 & 9 & $05: 38: 27.046$ & $-69: 07: 59.920$ & Obs. \\
\hline 278 & 22 & 9 & 05:38:23.309 & $-69: 07: 59.920$ & Obs. \\
\hline 279 & 23 & 9 & 05:38:19.565 & $-69: 07: 59.920$ & Obs. \\
\hline 280 & 24 & 9 & 05:38:15.830 & $-69: 07: 59.920$ & Obs. \\
\hline 281 & 25 & 9 & 05:38:12.096 & $-69: 07: 59.920$ & Obs. \\
\hline 282 & 26 & 9 & 05:38:08.359 & $-69: 07: 59.920$ & Obs. \\
\hline 283 & 27 & 9 & 05:38:04.615 & $-69: 07: 59.920$ & Obs. \\
\hline 284 & 28 & 9 & 05:38:00.881 & $-69: 07: 59.920$ & Obs. \\
\hline 285 & 29 & 9 & $05: 37: 57.146$ & $-69: 07: 59.920$ & Obs. \\
\hline 286 & 30 & 9 & $05: 37: 53.410$ & $-69: 07: 59.920$ & Obs. \\
\hline 287 & 31 & 9 & 05:37:49.666 & $-69: 07: 59.920$ & Obs. \\
\hline 288 & 32 & 9 & $05: 37: 45.929$ & $-69: 07: 59.920$ & Obs. \\
\hline 289 & 1 & 10 & 05:39:41.796 & $-69: 07: 39.860$ & Obs. \\
\hline 290 & 2 & 10 & 05:39:38.059 & $-69: 07: 39.940$ & Obs. \\
\hline 291 & 3 & 10 & 05:39:34.325 & $-69: 07: 39.940$ & Obs. \\
\hline 292 & 4 & 10 & 05:39:30.590 & $-69: 07: 39.940$ & Obs. \\
\hline 293 & 5 & 10 & 05:39:26.846 & $-69: 07: 39.940$ & Obs. \\
\hline 294 & 6 & 10 & 05:39:23.110 & $-69: 07: 39.940$ & Obs. \\
\hline 295 & 7 & 10 & 05:39:19.366 & $-69: 07: 39.860$ & Obs. \\
\hline 296 & 8 & 10 & 05:39:15.629 & $-69: 07: 39.940$ & Obs. \\
\hline 297 & 9 & 10 & 05:39:11.894 & $-69: 07: 39.860$ & Obs. \\
\hline 298 & 10 & 10 & 05:39:08.160 & $-69: 07: 39.940$ & Obs. \\
\hline 299 & 11 & 10 & 05:39:04.416 & $-69: 07: 39.940$ & Obs. \\
\hline 300 & 12 & 10 & 05:39:00.679 & $-69: 07: 39.940$ & Obs. \\
\hline 301 & 13 & 10 & 05:38:56.945 & $-69: 07: 39.940$ & Obs. \\
\hline 302 & 14 & 10 & 05:38:53.210 & $-69: 07: 39.940$ & Obs. \\
\hline 303 & 15 & 10 & $05: 38: 49.466$ & $-69: 07: 39.860$ & Obs. \\
\hline 304 & 16 & 10 & 05:38:45.730 & $-69: 07: 39.940$ & Obs. \\
\hline 305 & 17 & 10 & 05:38:41.995 & $-69: 07: 39.860$ & Obs. \\
\hline 306 & 18 & 10 & 05:38:38.261 & $-69: 07: 39.940$ & Obs. \\
\hline 307 & 19 & 10 & $05: 38: 34.512$ & $-69: 07: 40.080$ & No Obs. \\
\hline 308 & 20 & 10 & 05:38:30.780 & $-69: 07: 39.940$ & Obs. \\
\hline 309 & 21 & 10 & 05:38:27.048 & $-69: 07: 40.080$ & No Obs. \\
\hline 310 & 22 & 10 & 05:38:23.309 & $-69: 07: 39.940$ & Obs. \\
\hline 311 & 23 & 10 & 05:38:19.560 & $-69: 07: 40.080$ & No Obs. \\
\hline 312 & 24 & 10 & 05:38:15.830 & $-69: 07: 39.940$ & Obs. \\
\hline 313 & 25 & 10 & 05:38:12.096 & $-69: 07: 40.080$ & Obs. (IG) \\
\hline 314 & 26 & 10 & 05:38:08.359 & $-69: 07: 39.940$ & Obs. \\
\hline
\end{tabular}

Table A.1. continued.

\begin{tabular}{|c|c|c|c|c|c|}
\hline ID & $\begin{array}{c}X \\
\text { pix }\end{array}$ & $\begin{array}{c}Y \\
\text { pix }\end{array}$ & $\begin{array}{l}\text { RA (J2000) } \\
\text { h:m:s }\end{array}$ & $\begin{array}{c}\text { Dec (J2000) } \\
\text { d:m:s }\end{array}$ & Data \\
\hline 315 & 27 & 10 & $05: 38: 04.608$ & $-69: 07: 40.080$ & No Obs. \\
\hline 316 & 28 & 10 & 05:38:00.881 & $-69: 07: 39.940$ & Obs. \\
\hline 317 & 29 & 10 & 05:37:57.144 & $-69: 07: 40.080$ & No Obs. \\
\hline 318 & 30 & 10 & $05: 37: 53.410$ & $-69: 07: 39.940$ & Obs. \\
\hline 319 & 31 & 10 & 05:37:49.666 & $-69: 07: 39.860$ & Obs. \\
\hline 320 & 32 & 10 & $05: 37: 45.929$ & $-69: 07: 39.940$ & Obs. \\
\hline 321 & 1 & 11 & 05:39:41.796 & $-69: 07: 19.880$ & Obs. \\
\hline 322 & 2 & 11 & 05:39:38.059 & $-69: 07: 19.880$ & Obs. \\
\hline 323 & 3 & 11 & $05: 39: 34.320$ & $-69: 07: 19.880$ & Obs. \\
\hline 324 & 4 & 11 & 05:39:30.590 & $-69: 07: 19.880$ & Obs. \\
\hline 325 & 5 & 11 & 05:39:26.849 & $-69: 07: 19.880$ & Obs. \\
\hline 326 & 6 & 11 & $05: 39: 23.110$ & $-69: 07: 19.880$ & Obs. \\
\hline 327 & 7 & 11 & 05:39:19.366 & $-69: 07: 19.880$ & Obs. \\
\hline 328 & 8 & 11 & 05:39:15.629 & $-69: 07: 19.880$ & Obs. \\
\hline 329 & 9 & 11 & 05:39:11.894 & $-69: 07: 19.880$ & Obs. \\
\hline 330 & 10 & 11 & 05:39:08.160 & $-69: 07: 19.880$ & Obs. \\
\hline 331 & 11 & 11 & $05: 39$ & $-69: 07: 19.880$ & Obs. \\
\hline 332 & 12 & 11 & 05:39:00.679 & $-69: 07: 19.880$ & Obs. \\
\hline 333 & 13 & 11 & $05: 38: 56.950$ & $-69: 07: 19.880$ & Obs. \\
\hline 334 & 14 & 11 & 05:38:53.210 & $-69: 07: 19.880$ & Obs. \\
\hline 335 & 15 & 11 & 05:38:49.466 & $-69: 07: 19.880$ & Obs. \\
\hline 336 & 16 & 11 & $05: 38: 45.730$ & $-69: 07: 19.880$ & Obs. \\
\hline 337 & 17 & 11 & 05:38:41.995 & $-69: 07: 19.880$ & Obs. \\
\hline 338 & 18 & 11 & 05:38:38.261 & $-69: 07: 19.880$ & Obs. \\
\hline 339 & 19 & 11 & $05: 38: 34.514$ & $-69: 07: 19.880$ & Obs. \\
\hline 340 & 20 & 11 & $05: 38: 30.780$ & $-69: 07: 19.880$ & Obs. \\
\hline 341 & 21 & 11 & $05: 38: 27.050$ & $-69: 07: 19.880$ & Obs. \\
\hline 342 & 22 & 11 & 05:38:23.309 & $-69: 07: 19.880$ & Obs. \\
\hline 343 & 23 & 11 & 05:38:19.565 & $-69: 07: 19.880$ & Obs. \\
\hline 344 & 24 & 11 & $05: 38: 15.830$ & $-69: 07: 19.880$ & Obs. \\
\hline 345 & 25 & 11 & 05:38: & $-69: 07: 19.880$ & Obs. \\
\hline 346 & 26 & 11 & 05:38:08.359 & $-69: 07: 19.880$ & Obs. \\
\hline 347 & 27 & 11 & 05:38:04.615 & $-69: 07: 19.880$ & Obs. \\
\hline 348 & 28 & 11 & 05:38:00.881 & $-69: 07: 19.880$ & Obs. \\
\hline 349 & 29 & 11 & $05: 37: 57.146$ & $-69: 07: 19.880$ & Obs. \\
\hline 350 & 30 & 11 & $05: 37: 53.410$ & $-69: 07: 19.880$ & Obs. \\
\hline 351 & 31 & 11 & $05: 37: 49.666$ & $-69: 07: 19.880$ & Obs. \\
\hline 352 & 32 & 11 & $05: 37: 45.929$ & $-69: 07: 19.880$ & Obs. \\
\hline 353 & 1 & 12 & 05:39:41.796 & $-69: 06: 59.870$ & Obs. \\
\hline 354 & 2 & 12 & 05:39:38.059 & $-69: 06: 59.940$ & Obs. \\
\hline 355 & 3 & 12 & $05: 39: 34.320$ & $-69: 06: 59.940$ & Obs. \\
\hline 356 & 4 & 12 & 05:39:30.590 & $-69: 06: 59.940$ & Obs. \\
\hline 357 & 5 & 12 & 05:39:26.849 & $-69: 06: 59.940$ & Obs. \\
\hline 358 & 6 & 12 & 05:39:23.110 & $-69: 06: 59.940$ & Obs. \\
\hline 359 & 7 & 12 & 05:39:19.366 & $-69: 06: 59.870$ & Obs. \\
\hline 360 & 8 & 12 & $05:$ & $-69: 0$ & Obs. \\
\hline 361 & 9 & 12 & 05:39:11.894 & $-69: 06: 59.870$ & Obs. \\
\hline 362 & 10 & 12 & 05:39:08.160 & $-69: 06: 59.940$ & Obs. \\
\hline 363 & 11 & 12 & 05:39:04.421 & $-69: 06: 59.940$ & Obs. \\
\hline 364 & 12 & 12 & 05:39:00.679 & $-69: 06: 59.940$ & Obs. \\
\hline 365 & 13 & 12 & $05: 38: 56.950$ & $-69: 06: 59.940$ & Obs. \\
\hline 366 & 14 & 12 & 05:38:53.210 & $-69: 06: 59.940$ & Obs. \\
\hline 367 & 15 & 12 & 05:38:49.466 & $-69: 06: 59.870$ & Obs. \\
\hline 368 & 16 & 12 & $05: 38: 45.730$ & $-69: 06: 59.940$ & Obs. \\
\hline 369 & 17 & 12 & 05:38:41.995 & $-69: 06: 59.870$ & Obs. \\
\hline 370 & 18 & 12 & $05: 38: 38.261$ & $-69: 06: 59.940$ & Obs. \\
\hline 371 & 19 & 12 & $05: 38: 34.514$ & $-69: 06: 59.940$ & Obs. \\
\hline 372 & 20 & 12 & $05: 38: 30.780$ & $-69: 06: 59.940$ & Obs. \\
\hline 373 & 21 & 12 & $05: 38: 27.050$ & $-69: 06: 59.940$ & Obs. \\
\hline 374 & 22 & 12 & 05:38:23.309 & $-69: 06: 59.940$ & Obs. \\
\hline 375 & 23 & 12 & 05:38:19.565 & $-69: 06: 59.940$ & Obs. \\
\hline 376 & 24 & 12 & 05:38:15.830 & $-69: 06: 59.940$ & Obs. \\
\hline 377 & 25 & 12 & 05:38:12.096 & $-69: 06: 59.940$ & Obs. \\
\hline 378 & 26 & 12 & 05:38:08.359 & $-69: 06: 59.940$ & Obs. \\
\hline 379 & 27 & 12 & 05:38:04.615 & $-69: 06: 59.940$ & Obs. \\
\hline
\end{tabular}


Table A.1. continued.

\begin{tabular}{|c|c|c|c|c|c|}
\hline ID & $\begin{array}{c}X \\
\text { pix }\end{array}$ & $\begin{array}{c}Y \\
\text { pix }\end{array}$ & $\begin{array}{l}\text { RA (J2000) } \\
\text { h:m:s }\end{array}$ & $\begin{array}{c}\text { Dec (J2000) } \\
\text { d:m:s }\end{array}$ & Data \\
\hline 380 & 28 & 12 & $05: 38: 00.881$ & $-69: 06: 59.940$ & Obs. \\
\hline 381 & 29 & 12 & $05: 37: 57.146$ & $-69: 06: 59.940$ & Obs. \\
\hline 382 & 30 & 12 & $05: 37: 53.410$ & $-69: 06: 59.940$ & Obs. \\
\hline 383 & 31 & 12 & $05: 37: 49.666$ & $-69: 06: 59.870$ & Obs. \\
\hline 384 & 32 & 12 & $05: 37: 45.929$ & $-69: 06: 59.940$ & Obs. \\
\hline 385 & 1 & 13 & $05: 39: 41.796$ & $-69: 06: 39.890$ & Obs. \\
\hline 386 & 2 & 13 & 05:39:38.059 & $-69: 06: 39.890$ & Obs. \\
\hline 387 & 3 & 13 & $05: 39: 34.325$ & $-69: 06: 39.890$ & Obs. \\
\hline 388 & 4 & 13 & 05:39:30.590 & $-69: 06: 39.890$ & Obs. \\
\hline 389 & 5 & 13 & $05: 39: 26.846$ & $-69: 06: 39.890$ & Obs. \\
\hline 390 & 6 & 13 & $05: 39: 23.110$ & $-69: 06: 39.890$ & Obs. \\
\hline 391 & 7 & 13 & 05:39:19.366 & $-69: 06: 39.890$ & Obs. \\
\hline 392 & 8 & 13 & $05: 39: 15.629$ & $-69: 06: 39.890$ & Obs. \\
\hline 393 & 9 & 13 & 05:39:11.894 & $-69: 06: 39.890$ & Obs. \\
\hline 394 & 10 & 13 & $05: 39: 08.160$ & $-69: 06: 39.890$ & Obs. \\
\hline 395 & 11 & 13 & 05:39:04.416 & $-69: 06: 39.890$ & Obs. \\
\hline 396 & 12 & 13 & 05:39:00.679 & $-69: 06: 39.890$ & Obs. \\
\hline 397 & 13 & 13 & $05: 38: 56.945$ & $-69: 06: 39.890$ & Obs. \\
\hline 398 & 14 & 13 & $05: 38: 53.210$ & $-69: 06: 39.890$ & Obs. \\
\hline 399 & 15 & 13 & $05: 38: 49.466$ & $-69: 06: 39.890$ & Obs. \\
\hline 400 & 16 & 13 & $05: 38: 45.730$ & $-69: 06: 39.890$ & Obs. \\
\hline 401 & 17 & 13 & 05:38:41.995 & $-69: 06: 39.890$ & Obs. \\
\hline 402 & 18 & 13 & $05: 38: 38.261$ & $-69: 06: 39.890$ & Obs. \\
\hline 403 & 19 & 13 & $05: 38: 34.514$ & $-69: 06: 39.890$ & Obs. \\
\hline 404 & 20 & 13 & 05:38:30.780 & $-69: 06: 39.890$ & Obs. \\
\hline 405 & 21 & 13 & $05: 38: 27.050$ & $-69: 06: 39.890$ & Obs. \\
\hline 406 & 22 & 13 & 05:38:23.309 & $-69: 06: 39.890$ & Obs. \\
\hline 407 & 23 & 13 & $05: 38: 19.565$ & $-69: 06: 39.890$ & Obs. \\
\hline 408 & 24 & 13 & $05: 38: 15.830$ & $-69: 06: 39.890$ & Obs. \\
\hline 409 & 25 & 13 & 05:38:12.096 & $-69: 06: 39.890$ & Obs. \\
\hline 410 & 26 & 13 & 05:38:08.359 & $-69: 06: 39.890$ & Obs. \\
\hline 411 & 27 & 13 & 05:38:04.615 & $-69: 06: 39.890$ & Obs. \\
\hline 412 & 28 & 13 & 05:38:00.881 & $-69: 06: 39.890$ & Obs. \\
\hline 413 & 29 & 13 & $05: 37: 57.146$ & $-69: 06: 39.890$ & Obs. \\
\hline 414 & 30 & 13 & $05: 37: 53.410$ & $-69: 06: 39.890$ & Obs. \\
\hline 415 & 31 & 13 & $05: 37: 49.666$ & $-69: 06: 39.890$ & Obs. \\
\hline 416 & 32 & 13 & $05: 37: 45.929$ & $-69: 06: 39.890$ & Obs. \\
\hline 417 & 1 & 14 & 05:39:41.796 & $-69: 06: 19.870$ & Obs. \\
\hline 418 & 2 & 14 & 05:39:38.059 & $-69: 06: 19.940$ & Obs. \\
\hline 419 & 3 & 14 & $05: 39: 34.325$ & $-69: 06: 19.940$ & Obs. \\
\hline 420 & 4 & 14 & $05: 39: 30.590$ & $-69: 06: 19.940$ & Obs. \\
\hline 421 & 5 & 14 & $05: 39: 26.846$ & $-69: 06: 19.940$ & Obs. \\
\hline 422 & 6 & 14 & $05: 39: 23.110$ & $-69: 06: 19.940$ & Obs. \\
\hline 423 & 7 & 14 & $05: 39: 19.366$ & $-69: 06: 19.870$ & Obs. \\
\hline 424 & 8 & 14 & $05: 39: 15.629$ & $-69: 06: 19.940$ & Obs. \\
\hline 425 & 9 & 14 & 05:39:11.894 & $-69: 06: 19.870$ & Obs. \\
\hline 426 & 10 & 14 & 05:39:08.160 & $-69: 06: 19.940$ & Obs. \\
\hline 427 & 11 & 14 & 05:39:04.416 & $-69: 06: 19.940$ & Obs. \\
\hline 428 & 12 & 14 & 05:39:00.679 & $-69: 06: 19.940$ & Obs. \\
\hline 429 & 13 & 14 & 05:38:56.945 & $-69: 06: 19.940$ & Obs. \\
\hline 430 & 14 & 14 & $05: 38: 53.210$ & $-69: 06: 19.940$ & Obs. \\
\hline 431 & 15 & 14 & 05:38:49.464 & $-69: 06: 19.800$ & No Obs. \\
\hline 432 & 16 & 14 & $05: 38: 45.730$ & $-69: 06: 19.940$ & Obs. \\
\hline 433 & 17 & 14 & 05:38:41.976 & $-69: 06: 19.800$ & No Obs. \\
\hline 434 & 18 & 14 & $05: 38: 38.261$ & $-69: 06: 19.940$ & Obs. \\
\hline 435 & 19 & 14 & $05: 38: 34.514$ & $-69: 06: 19.940$ & Obs. \\
\hline 436 & 20 & 14 & $05: 38: 30.780$ & $-69: 06: 19.940$ & Obs. \\
\hline 437 & 21 & 14 & $05: 38: 27.050$ & $-69: 06: 19.940$ & Obs. \\
\hline 438 & 22 & 14 & 05:38:23.309 & $-69: 06: 19.940$ & Obs. \\
\hline 439 & 23 & 14 & $05: 38: 19.565$ & $-69: 06: 19.940$ & Obs. \\
\hline 440 & 24 & 14 & $05: 38: 15.830$ & $-69: 06: 19.940$ & Obs. \\
\hline 441 & 25 & 14 & 05:38:12.096 & $-69: 06: 19.940$ & Obs. \\
\hline 442 & 26 & 14 & 05:38:08.359 & $-69: 06: 19.940$ & Obs. \\
\hline 443 & 27 & 14 & 05:38:04.615 & $-69: 06: 19.940$ & Obs. \\
\hline
\end{tabular}

Table A.1. continued.

\begin{tabular}{|c|c|c|c|c|c|}
\hline ID & $\begin{array}{c}X \\
\text { pix }\end{array}$ & $\begin{array}{c}Y \\
\text { pix }\end{array}$ & $\begin{array}{l}\text { RA (J2000) } \\
\text { h:m:s }\end{array}$ & $\begin{array}{c}\operatorname{Dec}(\mathrm{J} 2000) \\
\mathrm{d}: \mathrm{m}: \mathrm{s}\end{array}$ & Data \\
\hline 444 & 28 & 14 & $05: 38: 00.881$ & $-69: 06: 19.940$ & Obs. \\
\hline 445 & 29 & 14 & 05:37:57.146 & $-69: 06: 19.940$ & Obs. \\
\hline 446 & 30 & 14 & 05:37:53.410 & $-69: 06: 19.940$ & Obs. \\
\hline 447 & 31 & 14 & $05: 37: 49.666$ & $-69: 06: 19.870$ & Obs. \\
\hline 448 & 32 & 14 & $05: 37: 45.929$ & $-69: 06: 19.940$ & Obs. \\
\hline 449 & 1 & 15 & 05:39:41.796 & $-69: 05: 59.890$ & Obs. \\
\hline 450 & 2 & 15 & 05:39:38.059 & 59.890 & Obs. \\
\hline 451 & 3 & 15 & 05:39:34.325 & $-69: 05: 59.890$ & Obs. \\
\hline 452 & 4 & 15 & 05:39:30.590 & $-69: 05: 59.890$ & Obs. \\
\hline 453 & 5 & 15 & $05: 39: 26.846$ & $-69: 05: 59.890$ & Obs. \\
\hline 454 & 6 & 15 & $05: 39: 23.110$ & $-69: 05: 59.890$ & Obs. \\
\hline 455 & 7 & 15 & 05:39:19.366 & $-69: 05: 59.890$ & Obs. \\
\hline 456 & 8 & 15 & 05:39:15.629 & $-69: 05: 59.890$ & Obs. \\
\hline 457 & 9 & 15 & 05:39:11.894 & $-69: 05: 59.890$ & Obs. \\
\hline 458 & 10 & 15 & 05:39:08.160 & $-69: 05: 59.890$ & Obs. \\
\hline 459 & 11 & 15 & 05:39:04.416 & $-69: 05: 59.890$ & Obs. \\
\hline 460 & 12 & 15 & 05:39:00.679 & $-69: 05: 59.890$ & Obs. \\
\hline 461 & 13 & 15 & 05:38:56.945 & $-69: 05: 59.890$ & Obs. \\
\hline 462 & 14 & 15 & $05: 3$ & $-69: 05: 59.890$ & Obs. \\
\hline 463 & 15 & 15 & .469 & $-69:$ & Obs. \\
\hline 464 & 16 & 15 & $05:$ & -69 : & Obs. \\
\hline 465 & 17 & 15 & $05: 3$ & $-69: 05: 59.890$ & Obs. \\
\hline 466 & 18 & 15 & $05: 3$ & $-69: 06: 00.000$ & No Obs. \\
\hline 467 & 19 & 15 & 4.514 & $-69: 05: 59.890$ & Obs. \\
\hline 468 & 20 & 15 & 05:38:30.780 & $-69: 05: 59.890$ & Obs. \\
\hline 469 & 21 & 15 & $05: 38: 27.046$ & $-69: 05: 59.890$ & Obs. \\
\hline 470 & 22 & 15 & 05:38:23.309 & $-69: 05: 59.890$ & Obs. \\
\hline 471 & 23 & 15 & 05:38:19.565 & $-69: 05: 59.890$ & Obs. \\
\hline 472 & 24 & 15 & 05:38:15.830 & $-69: 05: 59.890$ & Obs. \\
\hline 473 & 25 & 15 & 05:38:12.096 & $-69: 05: 59.890$ & Obs. \\
\hline 474 & 26 & 15 & 05:38:08.359 & $-69: 05: 59.890$ & Obs. \\
\hline 475 & 27 & 15 & 05:38:04.615 & $-69: 05: 59.890$ & Obs. \\
\hline 476 & 28 & 15 & 00.881 & $-69: 05$ & Obs. \\
\hline 477 & 29 & 15 & 46 & -69 & Obs. \\
\hline 478 & 30 & 15 & 3.410 & $-69:$ & Obs. \\
\hline 479 & 31 & 15 & :49.666 & $-69: 05: 59.890$ & Obs. \\
\hline 480 & 32 & 15 & $05: 37: 45.929$ & $-69: 05: 59.890$ & Obs. \\
\hline 481 & 1 & 16 & $05: 39: 41.796$ & $-69: 05: 39.880$ & Obs. \\
\hline 482 & 2 & 16 & $05: 39: 38.059$ & $-69: 05: 39.950$ & Obs. \\
\hline 483 & 3 & 16 & $05: 39: 34.325$ & $-69: 05: 39.950$ & Obs. \\
\hline 484 & 4 & 16 & $05: 39: 30.590$ & $-69: 05: 39.950$ & Obs. \\
\hline 485 & 5 & 16 & $05: 39: 26.846$ & $-69: 05: 39.950$ & Obs. \\
\hline 486 & 6 & 16 & $05: 39: 23.110$ & $-69: 05: 39.950$ & Obs. \\
\hline 487 & 7 & 16 & 05:39:19.366 & $-69: 05: 39.880$ & Obs. \\
\hline 488 & 8 & 16 & $05: 39: 15.629$ & $-69: 05: 39.950$ & Obs. \\
\hline 489 & 9 & 16 & 05:39:11.894 & $-69: 05: 39.880$ & Obs. \\
\hline 490 & 10 & 16 & 05:39:08.160 & $-69: 05: 39.950$ & Obs. \\
\hline 491 & 11 & 16 & 05: & $-69:$ & Obs. \\
\hline 492 & 12 & 16 & $05: 39: 00$ & $-69:$ & Obs. \\
\hline 493 & 13 & 16 & 05:38:56.945 & $-69: 05: 39.880$ & Obs. \\
\hline 494 & 14 & 16 & $05: 38: 53.210$ & $-69: 05: 39.950$ & Obs. \\
\hline 495 & 15 & 16 & $05: 38: 49.469$ & $-69: 05: 39.950$ & Obs. \\
\hline 496 & 16 & 16 & $05: 38: 45.730$ & $-69: 05: 39.950$ & Obs. \\
\hline 497 & 17 & 16 & $05: 38: 42.000$ & $-69: 05: 39.950$ & Obs. \\
\hline 498 & 18 & 16 & $05: 38: 38.256$ & $-69: 05: 39.840$ & No Obs. \\
\hline 499 & 19 & 16 & $05: 38: 34.514$ & $-69: 05: 39.880$ & Obs. \\
\hline 500 & 20 & 16 & $05: 38: 30.780$ & $-69: 05: 39.950$ & Obs. \\
\hline 501 & 21 & 16 & $05: 38: 27.046$ & $-69: 05: 39.880$ & Obs. \\
\hline 502 & 22 & 16 & 05:38:23.309 & $-69: 05: 39.950$ & Obs. \\
\hline 503 & 23 & 16 & $05: 38: 19.560$ & $-69: 05: 39.840$ & No Obs. \\
\hline 504 & 24 & 16 & $05: 38: 15.830$ & $-69: 05: 39.950$ & Obs. \\
\hline 505 & 25 & 16 & $05: 38: 12.096$ & $-69: 05: 39.880$ & Obs. \\
\hline 506 & 26 & 16 & 05:38:08.359 & $-69: 05: 39.950$ & Obs. \\
\hline 507 & 27 & 16 & $05: 38: 04.615$ & $-69: 05: 39.950$ & Obs. \\
\hline
\end{tabular}


S. Torres-Flores et al.: Studying the kinematics of the giant star-forming region 30 Doradus. I.

Table A.1. continued.

\begin{tabular}{|c|c|c|c|c|c|}
\hline ID & $\begin{array}{c}X \\
\text { pix }\end{array}$ & $\begin{array}{c}Y \\
\text { pix }\end{array}$ & $\begin{array}{c}\text { RA (J2000) } \\
\text { h:m:s }\end{array}$ & $\begin{array}{c}\text { Dec (J2000) } \\
\text { d:m:s }\end{array}$ & Data \\
\hline 508 & 28 & 16 & $05: 38: 00.881$ & $-69: 05: 39.950$ & Obs. \\
\hline 509 & 29 & 16 & $05: 37: 57.146$ & $-69: 05: 39.950$ & Obs. \\
\hline 510 & 30 & 16 & 05:37:53.410 & $-69: 05: 39.950$ & Obs. \\
\hline 511 & 31 & 16 & 05:37:49.666 & $-69: 05: 39.880$ & Obs. \\
\hline 512 & 32 & 16 & $05: 37: 45.929$ & $-69: 05: 39.950$ & Obs. \\
\hline 513 & 1 & 17 & 05:39:41.796 & $-69: 05: 19.900$ & Obs. \\
\hline 514 & 2 & 17 & 05:39:38.059 & $-69: 05: 19.900$ & Obs. \\
\hline 515 & 3 & 17 & $05: 39: 34.325$ & $-69: 05: 19.900$ & Obs. \\
\hline 516 & 4 & 17 & $05: 39: 30.590$ & $-69: 05: 19.900$ & Obs. \\
\hline 517 & 5 & 17 & $05: 39: 26.846$ & $-69: 05: 19.900$ & Obs. \\
\hline 518 & 6 & 17 & 05:39:23.110 & $-69: 05: 19.900$ & Obs. \\
\hline 519 & 7 & 17 & 05:39:19.366 & $-69: 05: 19.900$ & Obs. \\
\hline 520 & 8 & 17 & $05: 39: 15.629$ & $-69: 05: 19.900$ & Obs. \\
\hline 521 & 9 & 17 & 05:39:11.894 & $-69: 05: 19.900$ & Obs. \\
\hline 522 & 10 & 17 & 05:39:08.160 & $-69: 05: 19.900$ & Obs. \\
\hline 523 & 11 & 17 & 05:39:04.416 & $-69: 05: 19.900$ & Obs. \\
\hline 524 & 12 & 17 & 05:39:00.679 & $-69: 05: 19.900$ & Obs. \\
\hline 525 & 13 & 17 & 05:38:56.945 & $-69: 05: 19.900$ & Obs. \\
\hline 526 & 14 & 17 & $05: 38: 53.210$ & $-69: 05: 19.900$ & Obs. \\
\hline 527 & 15 & 17 & 05:38:49.469 & $-69: 05: 19.900$ & Obs. \\
\hline 528 & 16 & 17 & $05: 38: 45.730$ & $-69: 05: 19.900$ & Obs. \\
\hline 529 & 17 & 17 & $05: 38: 42.000$ & $-69: 05: 19.900$ & Obs. \\
\hline 530 & 18 & 17 & 05:38:38.261 & $-69: 05: 19.900$ & Obs. \\
\hline 531 & 19 & 17 & $05: 38: 34.514$ & $-69: 05: 19.900$ & Obs. \\
\hline 532 & 20 & 17 & 05:38:30.780 & $-69: 05: 19.900$ & Obs. \\
\hline 533 & 21 & 17 & $05: 38: 27.046$ & $-69: 05: 19.900$ & Obs. \\
\hline 534 & 22 & 17 & 05:38:23.309 & $-69: 05: 19.900$ & Obs. \\
\hline 535 & 23 & 17 & $05: 38: 19.560$ & $-69: 05: 20.040$ & No Obs. \\
\hline 536 & 24 & 17 & 05:38:16.032 & $-69: 05: 20.040$ & No Obs. \\
\hline 537 & 25 & 17 & 05:38:12.096 & $-69: 05: 19.900$ & Obs. \\
\hline 538 & 26 & 17 & 05:38:08.359 & $-69: 05: 19.900$ & Obs. \\
\hline 539 & 27 & 17 & 05:38:04.608 & $-69: 05: 20.040$ & No Obs. \\
\hline 540 & 28 & 17 & 05:38:00.888 & $-69: 05: 19.680$ & Obs. (IG) \\
\hline 541 & 29 & 17 & $05: 37: 57.146$ & $-69: 05: 19.900$ & Obs. \\
\hline 542 & 30 & 17 & 05:37:53.410 & $-69: 05: 19.900$ & Obs. \\
\hline 543 & 31 & 17 & 05:37:49.666 & $-69: 05: 19.900$ & Obs. \\
\hline 544 & 32 & 17 & $05: 37: 45.929$ & $-69: 05: 19.900$ & Obs. \\
\hline 545 & 1 & 18 & 05:39:41.796 & $-69: 04: 59.880$ & Obs. \\
\hline 546 & 2 & 18 & 05:39:38.059 & $-69: 04: 59.920$ & Obs. \\
\hline 547 & 3 & 18 & 05:39:34.325 & $-69: 04: 59.920$ & Obs. \\
\hline 548 & 4 & 18 & $05: 39: 30.590$ & $-69: 04: 59.920$ & Obs. \\
\hline 549 & 5 & 18 & 05:39:26.846 & $-69: 04: 59.920$ & Obs. \\
\hline 550 & 6 & 18 & 05:39:23.110 & $-69: 04: 59.920$ & Obs. \\
\hline 551 & 7 & 18 & 05:39:19.368 & $-69: 04: 59.880$ & No Obs. \\
\hline 552 & 8 & 18 & 05:39:15.629 & $-69: 04: 59.920$ & Obs. \\
\hline 553 & 9 & 18 & 05:39:11.880 & $-69: 04: 59.880$ & No Obs. \\
\hline 554 & 10 & 18 & 05:39:08.160 & $-69: 04: 59.920$ & Obs. \\
\hline 555 & 11 & 18 & 05:39:04.416 & $-69: 04: 59.880$ & No Obs. \\
\hline 556 & 12 & 18 & 05:39:00.679 & $-69: 04: 59.920$ & Obs. \\
\hline 557 & 13 & 18 & $05: 38: 56.928$ & $-69: 04: 59.880$ & Obs. (IG) \\
\hline 558 & 14 & 18 & 05:38:53.210 & $-69: 04: 59.920$ & Obs. \\
\hline 559 & 15 & 18 & $05: 38: 49.466$ & $-69: 04: 59.880$ & Obs. \\
\hline 560 & 16 & 18 & $05: 38: 45.730$ & $-69: 04: 59.920$ & Obs. \\
\hline 561 & 17 & 18 & 05:38:41.995 & $-69: 04: 59.880$ & Obs. \\
\hline 562 & 18 & 18 & 05:38:38.261 & $-69: 04: 59.920$ & Obs. \\
\hline 563 & 19 & 18 & $05: 38: 34.512$ & $-69: 04: 59.880$ & Obs. (IG) \\
\hline 564 & 20 & 18 & 05:38:30.780 & $-69: 04: 59.920$ & Obs. \\
\hline 565 & 21 & 18 & 05:38:27.048 & $-69: 04: 59.880$ & No Obs. \\
\hline 566 & 22 & 18 & 05:38:23.309 & $-69: 04: 59.920$ & Obs. \\
\hline 567 & 23 & 18 & 05:38:19.565 & $-69: 04: 59.880$ & Obs. \\
\hline 568 & 24 & 18 & $05: 38: 15.816$ & $-69: 04: 59.880$ & No Obs. \\
\hline 569 & 25 & 18 & 05:38:12.312 & $-69: 05: 00.240$ & No Obs. \\
\hline 570 & 26 & 18 & 05:38:08.359 & $-69: 04: 59.920$ & Obs. \\
\hline 571 & 27 & 18 & 05:38:04.608 & $-69: 04: 59.880$ & No Obs. \\
\hline 572 & 28 & 18 & 05:38:01.104 & $-69: 05: 00.240$ & No Obs. \\
\hline
\end{tabular}

Table A.1. continued.

\begin{tabular}{|c|c|c|c|c|c|}
\hline ID & $\begin{array}{c}X \\
\text { pix }\end{array}$ & $\begin{array}{c}Y \\
\text { pix }\end{array}$ & $\begin{array}{l}\text { RA (J2000) } \\
\text { h:m:s }\end{array}$ & $\begin{array}{c}\text { Dec (J2000) } \\
\text { d:m:s }\end{array}$ & Data \\
\hline 573 & 29 & 18 & $05: 37: 57.146$ & $-69: 04: 59.920$ & Obs. \\
\hline 574 & 30 & 18 & $05: 37: 53.410$ & $-69: 04: 59.920$ & Obs. \\
\hline 575 & 31 & 18 & $05: 37: 49.666$ & $-69: 04: 59.880$ & Obs. \\
\hline 576 & 32 & 18 & $05: 37: 45.929$ & $-69: 04: 59.920$ & Obs. \\
\hline 577 & 1 & 19 & $05: 39: 41.796$ & $-69: 04: 39.900$ & Obs. \\
\hline 578 & 2 & 19 & 05:39:38.059 & $-69: 04: 39.900$ & Obs. \\
\hline 579 & 3 & 19 & $05: 39: 34.325$ & $-69: 04: 39.900$ & Obs. \\
\hline 580 & 4 & 19 & $05: 39: 30.590$ & $-69: 04: 39.900$ & Obs. \\
\hline 581 & 5 & 19 & $05: 39: 26.846$ & $-69: 04: 39.900$ & Obs. \\
\hline 582 & 6 & 19 & 05:39:23.110 & $-69: 04: 39.900$ & Obs. \\
\hline 583 & 7 & 19 & $05: 39: 19.366$ & $-69: 04: 39.900$ & Obs. \\
\hline 584 & 8 & 19 & $05: 39: 15.629$ & $-69: 04: 39.900$ & Obs. \\
\hline 585 & 9 & 19 & 05:39:11.894 & $-69: 04: 39.900$ & Obs. \\
\hline 586 & 10 & 19 & 05:39:08.160 & $-69: 04: 39.900$ & Obs. \\
\hline 587 & 11 & 19 & 05:39:04.421 & $-69: 04: 39.900$ & Obs. \\
\hline 588 & 12 & 19 & 05:39:00.679 & $-69: 04: 39.900$ & Obs. \\
\hline 589 & 13 & 19 & $05: 38: 56.945$ & $-69: 04: 39.900$ & Obs. \\
\hline 590 & 14 & 19 & $05: 38: 53.210$ & $-69: 04: 39.900$ & Obs. \\
\hline 591 & 15 & 19 & 05:38:49.466 & $-69: 04: 39.900$ & Obs. \\
\hline 592 & 16 & 19 & 05:38:45.730 & $-69: 04: 39.900$ & Obs. \\
\hline 593 & 17 & 19 & 05:38:41.995 & $-69: 04: 39.900$ & Obs. \\
\hline 594 & 18 & 19 & $05: 38: 38.261$ & $-69: 04: 39.900$ & Obs. \\
\hline 595 & 19 & 19 & $05: 38: 34.514$ & $-69: 04: 39.900$ & Obs. \\
\hline 596 & 20 & 19 & $05: 38: 30.780$ & $-69: 04: 39.900$ & Obs. \\
\hline 597 & 21 & 19 & $05: 38: 27.046$ & $-69: 04: 39.900$ & Obs. \\
\hline 598 & 22 & 19 & 05:38:23.309 & $-69: 04: 39.900$ & Obs. \\
\hline 599 & 23 & 19 & 05:38:19.565 & $-69: 04: 39.900$ & Obs. \\
\hline 600 & 24 & 19 & $05: 38: 15.830$ & $-69: 04: 39.900$ & Obs. \\
\hline 601 & 25 & 19 & 05:38:12.096 & $-69: 04: 40.080$ & No Obs. \\
\hline 602 & 26 & 19 & 05:38:08.568 & $-69: 04: 40.080$ & No Obs. \\
\hline 603 & 27 & 19 & 05:38:04.848 & $-69: 04: 40.080$ & Obs. (IG) \\
\hline 604 & 28 & 19 & 05:38:00.888 & $-69: 04: 39.720$ & No Obs. \\
\hline 605 & 29 & 19 & $05: 37: 57.146$ & $-69: 04: 39.900$ & Obs. \\
\hline 606 & 30 & 19 & 05:37:53.410 & $-69: 04: 39.900$ & Obs. \\
\hline 607 & 31 & 19 & 05:37:49.666 & $-69: 04: 39.900$ & Obs. \\
\hline 608 & 32 & 19 & $05: 37: 45.929$ & $-69: 04: 39.900$ & Obs. \\
\hline 609 & 1 & 20 & 05:39:41.796 & $-69: 04: 19.880$ & Obs. \\
\hline 610 & 2 & 20 & $05: 39: 38.059$ & $-69: 04: 19.920$ & Obs. \\
\hline 611 & 3 & 20 & $05: 39: 34.325$ & $-69: 04: 19.920$ & Obs. \\
\hline 612 & 4 & 20 & $05: 39: 30.590$ & $-69: 04: 19.920$ & Obs. \\
\hline 613 & 5 & 20 & 05:39:26.846 & $-69: 04: 19.920$ & Obs. \\
\hline 614 & 6 & 20 & 05:39:23.110 & $-69: 04: 19.920$ & Obs. \\
\hline 615 & 7 & 20 & 05:39:19.366 & $-69: 04: 19.920$ & Obs. \\
\hline 616 & 8 & 20 & $05: 39: 15.629$ & $-69: 04: 19.920$ & Obs. \\
\hline 617 & 9 & 20 & 05:39:11.894 & $-69: 04: 19.920$ & Obs. \\
\hline 618 & 10 & 20 & 05:39:08.160 & $-69: 04: 19.920$ & Obs. \\
\hline 619 & 11 & 20 & 05:39:04.421 & $-69: 04: 19.920$ & Obs. \\
\hline 620 & 12 & 20 & 05:39:00.679 & $-69: 04: 19.920$ & Obs. \\
\hline 621 & 13 & 20 & $05: 38: 56.945$ & $-69: 04: 19.920$ & Obs. \\
\hline 622 & 14 & 20 & $05: 38: 53.210$ & $-69: 04: 19.920$ & Obs. \\
\hline 623 & 15 & 20 & $05: 38: 49.466$ & $-69: 04: 19.880$ & Obs. \\
\hline 624 & 16 & 20 & $05: 38: 45.730$ & $-69: 04: 19.920$ & Obs. \\
\hline 625 & 17 & 20 & 05:38:41.995 & $-69: 04: 19.880$ & Obs. \\
\hline 626 & 18 & 20 & 05:38:38.261 & $-69: 04: 19.920$ & Obs. \\
\hline 627 & 19 & 20 & $05: 38: 34.514$ & $-69: 04: 19.920$ & Obs. \\
\hline 628 & 20 & 20 & 05:38:30.780 & $-69: 04: 19.920$ & Obs. \\
\hline 629 & 21 & 20 & 05:38:27.046 & $-69: 04: 19.920$ & Obs. \\
\hline 630 & 22 & 20 & 05:38:23.309 & $-69: 04: 19.920$ & Obs. \\
\hline 631 & 23 & 20 & $05: 38: 19.565$ & $-69: 04: 19.880$ & Obs. \\
\hline 632 & 24 & 20 & $05: 38: 15.830$ & $-69: 04: 19.920$ & Obs. \\
\hline 633 & 25 & 20 & 05:38:12.096 & $-69: 04: 19.880$ & Obs. \\
\hline 634 & 26 & 20 & 05:38:08.352 & $-69: 04: 19.920$ & No Obs. \\
\hline 635 & 27 & 20 & 05:38:04.848 & $-69: 04: 20.280$ & No Obs. \\
\hline 636 & 28 & 20 & 05:38:00.888 & $-69: 04: 19.920$ & No Obs. \\
\hline 637 & 29 & 20 & 05:37:57.146 & $-69: 04: 19.920$ & Obs. \\
\hline
\end{tabular}


Table A.1. continued.

\begin{tabular}{|c|c|c|c|c|c|}
\hline ID & $\begin{array}{c}X \\
\text { pix }\end{array}$ & $\begin{array}{c}Y \\
\text { pix }\end{array}$ & $\begin{array}{c}\text { RA (J2000) } \\
\text { h:m:s }\end{array}$ & $\begin{array}{c}\operatorname{Dec}(\mathrm{J} 2000) \\
\mathrm{d}: \mathrm{m}: \mathrm{s}\end{array}$ & Data \\
\hline 638 & 30 & 20 & $05: 37: 53.410$ & $-69: 04: 19.920$ & Obs. \\
\hline 639 & 31 & 20 & 05:37:49.666 & $-69: 04: 19.880$ & Obs. \\
\hline 640 & 32 & 20 & $05: 37: 45.929$ & $-69: 04: 19.920$ & Obs. \\
\hline 641 & 1 & 21 & 05:39:41.796 & $-69: 03: 59.900$ & Obs. \\
\hline 642 & 2 & 21 & 05:39:38.059 & $-69: 03: 59.900$ & Obs. \\
\hline 643 & 3 & 21 & 05:39:34.325 & $-69: 03: 59.900$ & Obs. \\
\hline 644 & 4 & 21 & $05: 39: 30.590$ & $-69: 03: 59.900$ & Obs. \\
\hline 645 & 5 & 21 & $05: 39: 26.846$ & $-69: 03: 59.900$ & Obs. \\
\hline 646 & 6 & 21 & 05:39:23.110 & $-69: 03: 59.900$ & Obs. \\
\hline 647 & 7 & 21 & 05:39:19.366 & $-69: 03: 59.900$ & Obs. \\
\hline 648 & 8 & 21 & 05:39:15.629 & $-69: 03: 59.900$ & Obs. \\
\hline 649 & 9 & 21 & 05:39:11.894 & $-69: 03: 59.900$ & Obs. \\
\hline 650 & 10 & 21 & 05:39:08.160 & $-69: 03: 59.900$ & Obs. \\
\hline 651 & 11 & 21 & 05:39:04.421 & $-69: 03: 59.900$ & Obs. \\
\hline 652 & 12 & 21 & 05:39:00.679 & $-69: 03: 59.900$ & Obs. \\
\hline 653 & 13 & 21 & $05: 38: 56.945$ & $-69: 03: 59.900$ & Obs. \\
\hline 654 & 14 & 21 & 05:38:53.210 & $-69: 03: 59.900$ & Obs. \\
\hline 655 & 15 & 21 & 05:38:49.466 & $-69: 03: 59.900$ & Obs. \\
\hline 656 & 16 & 21 & 05:38:45.730 & $-69: 03: 59.900$ & Obs. \\
\hline 657 & 17 & 21 & 05:38:41.995 & $-69: 03: 59.900$ & Obs. \\
\hline 658 & 18 & 21 & 05:38:38.261 & $-69: 03: 59.900$ & Obs. \\
\hline 659 & 19 & 21 & $05: 38: 34.519$ & $-69: 03: 59.900$ & Obs. \\
\hline 660 & 20 & 21 & 05:38:30.780 & $-69: 03: 59.900$ & Obs. \\
\hline 661 & 21 & 21 & $05: 38: 27.050$ & $-69: 03: 59.900$ & Obs. \\
\hline 662 & 22 & 21 & 05:38:23.309 & $-69: 03: 59.900$ & Obs. \\
\hline 663 & 23 & 21 & 05:38:19.565 & $-69: 03: 59.900$ & Obs. \\
\hline 664 & 24 & 21 & 05:38:15.830 & $-69: 03: 59.900$ & Obs. \\
\hline 665 & 25 & 21 & 05:38:12.096 & $-69: 03: 59.900$ & Obs. \\
\hline 666 & 26 & 21 & 05:38:08.359 & $-69: 03: 59.900$ & Obs. \\
\hline 667 & 27 & 21 & 05:38:04.620 & $-69: 03: 59.900$ & Obs. \\
\hline 668 & 28 & 21 & 05:38:00.881 & $-69: 03: 59.900$ & Obs. \\
\hline 669 & 29 & 21 & 05:37:57.139 & $-69: 03: 59.900$ & Obs. \\
\hline 670 & 30 & 21 & $05: 37: 53.410$ & $-69: 03: 59.900$ & Obs. \\
\hline 671 & 31 & 21 & 05:37:49.666 & $-69: 03: 59.900$ & Obs. \\
\hline 672 & 32 & 21 & 05:37:45.929 & $-69: 03: 59.900$ & Obs. \\
\hline 673 & 1 & 22 & 05:39:41.796 & $-69: 03: 39.850$ & Obs. \\
\hline 674 & 2 & 22 & 05:39:38.059 & $-69: 03: 39.920$ & Obs. \\
\hline 675 & 3 & 22 & $05: 39: 34.325$ & $-69: 03: 39.920$ & Obs. \\
\hline 676 & 4 & 22 & 05:39:30.590 & $-69: 03: 39.920$ & Obs. \\
\hline 677 & 5 & 22 & 05:39:26.846 & $-69: 03: 39.920$ & Obs. \\
\hline 678 & 6 & 22 & 05:39:23.110 & $-69: 03: 39.920$ & Obs. \\
\hline 679 & 7 & 22 & 05:39:19.366 & $-69: 03: 39.920$ & Obs. \\
\hline 680 & 8 & 22 & 05:39:15.629 & $-69: 03: 39.920$ & Obs. \\
\hline 681 & 9 & 22 & 05:39:11.894 & $-69: 03: 39.920$ & Obs. \\
\hline 682 & 10 & 22 & 05:39:08.160 & $-69: 03: 39.920$ & Obs. \\
\hline 683 & 11 & 22 & 05:39:04.421 & $-69: 03: 39.920$ & Obs. \\
\hline 684 & 12 & 22 & 05:39:00.679 & $-69: 03: 39.920$ & Obs. \\
\hline 685 & 13 & 22 & 05:38:56.945 & $-69: 03: 39.920$ & Obs. \\
\hline 686 & 14 & 22 & 05:38:53.210 & $-69: 03: 39.920$ & Obs. \\
\hline 687 & 15 & 22 & 05:38:49.466 & $-69: 03: 39.850$ & Obs. \\
\hline 688 & 16 & 22 & 05:38:45.730 & $-69: 03: 39.920$ & Obs. \\
\hline 689 & 17 & 22 & 05:38:41.995 & $-69: 03: 39.850$ & Obs. \\
\hline 690 & 18 & 22 & 05:38:38.261 & $-69: 03: 39.920$ & Obs. \\
\hline 691 & 19 & 22 & 05:38:34.519 & $-69: 03: 39.920$ & Obs. \\
\hline 692 & 20 & 22 & 05:38:30.780 & $-69: 03: 39.920$ & Obs. \\
\hline 693 & 21 & 22 & $05: 38: 27.050$ & $-69: 03: 39.920$ & Obs. \\
\hline 694 & 22 & 22 & 05:38:23.309 & $-69: 03: 39.920$ & Obs. \\
\hline 695 & 23 & 22 & 05:38:19.565 & $-69: 03: 39.850$ & Obs. \\
\hline 696 & 24 & 22 & 05:38:15.830 & $-69: 03: 39.920$ & Obs. \\
\hline 697 & 25 & 22 & 05:38:12.096 & $-69: 03: 39.850$ & Obs. \\
\hline 698 & 26 & 22 & 05:38:08.359 & $-69: 03: 39.920$ & Obs. \\
\hline 699 & 27 & 22 & 05:38:04.620 & $-69: 03: 39.920$ & Obs. \\
\hline 700 & 28 & 22 & 05:38:00.881 & $-69: 03: 39.920$ & Obs. \\
\hline 701 & 29 & 22 & 05:37:57.139 & $-69: 03: 39.920$ & Obs. \\
\hline 702 & 30 & 22 & 05:37:53.410 & $-69: 03: 39.920$ & Obs. \\
\hline
\end{tabular}

Table A.1. continued.

\begin{tabular}{|c|c|c|c|c|c|}
\hline ID & $\begin{array}{c}X \\
\text { pix }\end{array}$ & $\begin{array}{c}Y \\
\text { pix }\end{array}$ & $\begin{array}{l}\text { RA (J2000) } \\
\text { h:m:s }\end{array}$ & $\begin{array}{c}\operatorname{Dec}(\mathrm{J} 2000) \\
\mathrm{d}: \mathrm{m}: \mathrm{s}\end{array}$ & Data \\
\hline 703 & 31 & 22 & $05: 37: 49.666$ & $-69: 03: 39.850$ & Obs. \\
\hline 704 & 32 & 22 & $05: 37: 45.929$ & $-69: 03: 39.920$ & Obs. \\
\hline 705 & 1 & 23 & 05:39:41.796 & $-69: 03: 19.910$ & Obs. \\
\hline 706 & 2 & 23 & 05:39:38.059 & $-69: 03: 19.910$ & Obs. \\
\hline 707 & 3 & 23 & $05: 39: 34.325$ & $-69: 03: 19.910$ & Obs. \\
\hline 708 & 4 & 23 & $05: 39: 30.590$ & $-69: 03: 19.910$ & Obs. \\
\hline 709 & 5 & 23 & $05: 39: 26.846$ & $-69: 03: 19.910$ & Obs. \\
\hline 710 & 6 & 23 & 05:39:23.110 & $-69: 03: 19.910$ & Obs. \\
\hline 711 & 7 & 23 & 05:39:19.366 & $-69: 03: 19.910$ & Obs. \\
\hline 712 & 8 & 23 & $05: 39: 15.629$ & $-69: 03: 19.910$ & Obs. \\
\hline 713 & 9 & 23 & $05: 39: 11.894$ & $-69: 03: 19.910$ & Obs. \\
\hline 714 & 10 & 23 & 05:39:08.160 & $-69: 03: 19.910$ & Obs. \\
\hline 715 & 11 & 23 & 05:39:04.416 & $-69: 03: 19.910$ & Obs. \\
\hline 716 & 12 & 23 & 05:39:00.679 & $-69: 03: 19.910$ & Obs. \\
\hline 717 & 13 & 23 & 05:38:56.945 & $-69: 03: 19.910$ & Obs. \\
\hline 718 & 14 & 23 & 05:38:53.210 & $-69: 03: 19.910$ & Obs. \\
\hline 719 & 15 & 23 & $05: 38: 49.466$ & $-69: 03: 19.910$ & Obs. \\
\hline 720 & 16 & 23 & $05: 3 \varepsilon$ & $-69:$ & Obs. \\
\hline 721 & 17 & 23 & .995 & $-69:$ & Obs. \\
\hline 722 & 18 & 23 & 05:38: & $-69:($ & Obs. \\
\hline 723 & 19 & 23 & $05: 38: 34.514$ & $-69: 03: 19.910$ & Obs. \\
\hline 724 & 20 & 23 & 05:38:30.780 & $-69: 03: 19.910$ & Obs. \\
\hline 725 & 21 & 23 & $05: 38: 27.046$ & $-69: 03: 19.910$ & Obs. \\
\hline 726 & 22 & 23 & 05:38:23.309 & $-69: 03: 19.910$ & Obs. \\
\hline 727 & 23 & 23 & $05: 38: 19.565$ & $-69: 03: 19.910$ & Obs. \\
\hline 728 & 24 & 23 & $05: 38: 15.830$ & $-69: 03: 19.910$ & Obs. \\
\hline 729 & 25 & 23 & 05:38:12.096 & $-69: 03: 19.910$ & Obs. \\
\hline 730 & 26 & 23 & 05:38:08.359 & $-69: 03: 19.910$ & Obs. \\
\hline 731 & 27 & 23 & 05:38:04.615 & $-69: 03: 19.910$ & Obs. \\
\hline 732 & 28 & 23 & 05:38:00.881 & $-69: 03: 19.910$ & Obs. \\
\hline 733 & 29 & 23 & $05: 37: 57.146$ & $-69: 03: 19.910$ & Obs. \\
\hline 734 & 30 & 23 & $05: 37: 53.410$ & $-69: 03:$ & Obs. \\
\hline 735 & 31 & 23 & 9.666 & $-69:$ & Obs. \\
\hline 736 & 32 & 23 & $05: 3$ & $-69:($ & Obs. \\
\hline 737 & 1 & 24 & 05:39:41.796 & $-69: 02: 59.860$ & Obs. \\
\hline 738 & 2 & 24 & 05:39:38.059 & $-69: 02: 59.930$ & Obs. \\
\hline 739 & 3 & 24 & $05: 39: 34.325$ & $-69: 02: 59.860$ & Obs. \\
\hline 740 & 4 & 24 & $05: 39: 30.590$ & $-69: 02: 59.930$ & Obs. \\
\hline 741 & 5 & 24 & $05: 39: 26.846$ & $-69: 02: 59.860$ & Obs. \\
\hline 742 & 6 & 24 & $05: 39: 23.110$ & $-69: 02: 59.930$ & Obs. \\
\hline 743 & 7 & 24 & $05: 39: 19.366$ & $-69: 02: 59.860$ & Obs. \\
\hline 744 & 8 & 24 & $05: 39: 15.629$ & $-69: 02: 59.930$ & Obs. \\
\hline 745 & 9 & 24 & 05:39:11.894 & $-69: 02: 59.860$ & Obs. \\
\hline 746 & 10 & 24 & 05:39:08.160 & $-69: 02: 59.930$ & Obs. \\
\hline 747 & 11 & 24 & 05:39:04.416 & $-69: 03: 00.000$ & No Obs. \\
\hline 748 & 12 & 24 & 05:39:00.679 & $-69: 02: 59.930$ & Obs. \\
\hline 749 & 13 & 24 & $05: 38: 56.945$ & $-69: 02: 59.860$ & Obs. \\
\hline 750 & 14 & 24 & 10 & $-69:$ & Obs. \\
\hline 751 & 15 & 24 & $05: 3$ & $-69:($ & Obs. \\
\hline 752 & 16 & 24 & $05: 38: 45.730$ & $-69: 02: 59.930$ & Obs. \\
\hline 753 & 17 & 24 & $05: 38: 41.995$ & $-69: 02: 59.860$ & Obs. \\
\hline 754 & 18 & 24 & $05: 38: 38.261$ & $-69: 02: 59.930$ & Obs. \\
\hline 755 & 19 & 24 & $05: 38: 34.514$ & $-69: 02: 59.930$ & Obs. \\
\hline 756 & 20 & 24 & $05: 38: 30.780$ & $-69: 02: 59.930$ & Obs. \\
\hline 757 & 21 & 24 & $05: 38: 27.046$ & $-69: 02: 59.930$ & Obs. \\
\hline 758 & 22 & 24 & 05:38:23.309 & $-69: 02: 59.930$ & Obs. \\
\hline 759 & 23 & 24 & $05: 38: 19.565$ & $-69: 02: 59.860$ & Obs. \\
\hline 760 & 24 & 24 & $05: 38: 15.830$ & $-69: 02: 59.930$ & Obs. \\
\hline 761 & 25 & 24 & 05:38:12.096 & $-69: 02: 59.860$ & Obs. \\
\hline 762 & 26 & 24 & 05:38:08.359 & $-69: 02: 59.930$ & Obs. \\
\hline 763 & 27 & 24 & 05:38:04.615 & $-69: 02: 59.930$ & Obs. \\
\hline 764 & 28 & 24 & 05:38:00.881 & $-69: 02: 59.930$ & Obs. \\
\hline 765 & 29 & 24 & $05: 37: 57.146$ & $-69: 02: 59.930$ & Obs. \\
\hline 766 & 30 & 24 & 05:37:53.410 & $-69: 02: 59.930$ & Obs. \\
\hline 767 & 31 & 24 & 05:37:49.666 & $-69: 02: 59.860$ & Obs. \\
\hline
\end{tabular}


S. Torres-Flores et al.: Studying the kinematics of the giant star-forming region 30 Doradus. I.

Table A.1. continued.

\begin{tabular}{|c|c|c|c|c|c|}
\hline ID & $\begin{array}{c}X \\
\text { pix }\end{array}$ & $\begin{array}{c}Y \\
\text { pix }\end{array}$ & $\begin{array}{l}\text { RA (J2000) } \\
\text { h:m:s }\end{array}$ & $\begin{array}{c}\text { Dec (J2000) } \\
\text { d:m:s }\end{array}$ & Data \\
\hline 768 & 32 & 24 & $05: 37: 45.929$ & $-69: 02: 59.930$ & Obs. \\
\hline 769 & 1 & 25 & $05: 39: 41.796$ & $-69: 02: 39.910$ & Obs. \\
\hline 770 & 2 & 25 & $05: 39: 38.059$ & $-69: 02: 39.910$ & Obs. \\
\hline 771 & 3 & 25 & 05:39:34.325 & $-69: 02: 39.910$ & Obs. \\
\hline 772 & 4 & 25 & 05:39:30.590 & $-69: 02: 39.910$ & Obs. \\
\hline 773 & 5 & 25 & $05: 39: 26.846$ & $-69: 02: 39.910$ & Obs. \\
\hline 774 & 6 & 25 & 05:39:23.110 & $-69: 02: 39.910$ & Obs. \\
\hline 775 & 7 & 25 & 05:39:19.366 & $-69: 02: 39.910$ & Obs. \\
\hline 776 & 8 & 25 & 05:39:15.629 & $-69: 02: 39.910$ & Obs. \\
\hline 777 & 9 & 25 & 05:39:11.894 & $-69: 02: 39.910$ & Obs. \\
\hline 778 & 10 & 25 & 05:39:08.160 & $-69: 02: 39.910$ & Obs. \\
\hline 779 & 11 & 25 & 05:39:04.416 & $-69: 02: 39.840$ & No Obs. \\
\hline 780 & 12 & 25 & 05:39:00.672 & $-69: 02: 39.840$ & No Obs. \\
\hline 781 & 13 & 25 & 05:38:56.945 & $-69: 02: 39.910$ & Obs. \\
\hline 782 & 14 & 25 & $05: 38: 53.210$ & $-69: 02: 39.910$ & Obs. \\
\hline 783 & 15 & 25 & 05:38:49.466 & $-69: 02: 39.910$ & Obs. \\
\hline 784 & 16 & 25 & $05: 38: 45.730$ & $-69: 02: 39.910$ & Obs. \\
\hline 785 & 17 & 25 & 05:38:41.995 & $-69: 02: 39.910$ & Obs. \\
\hline 786 & 18 & 25 & $05: 38: 38.261$ & $-69: 02: 39.910$ & Obs. \\
\hline 787 & 19 & 25 & $05: 38: 34.514$ & $-69: 02: 39.910$ & Obs. \\
\hline 788 & 20 & 25 & 05:38:30.780 & $-69: 02: 39.910$ & Obs. \\
\hline 789 & 21 & 25 & $05: 38: 27.046$ & $-69: 02: 39.910$ & Obs. \\
\hline 790 & 22 & 25 & 05:38:23.309 & $-69: 02: 39.910$ & Obs. \\
\hline 791 & 23 & 25 & 05:38:19.565 & $-69: 02: 39.910$ & Obs. \\
\hline 792 & 24 & 25 & $05: 38: 15.830$ & $-69: 02: 39.910$ & Obs. \\
\hline 793 & 25 & 25 & 05:38:12.096 & $-69: 02: 39.910$ & Obs. \\
\hline 794 & 26 & 25 & 05:38:08.359 & $-69: 02: 39.910$ & Obs. \\
\hline 795 & 27 & 25 & 05:38:04.615 & $-69: 02: 39.910$ & Obs. \\
\hline 796 & 28 & 25 & 05:38:00.881 & $-69: 02: 39.910$ & Obs. \\
\hline 797 & 29 & 25 & $05: 37: 57.146$ & $-69: 02: 39.910$ & Obs. \\
\hline 798 & 30 & 25 & $05: 37: 53.410$ & $-69: 02: 39.910$ & Obs. \\
\hline 799 & 31 & 25 & 05:37:49.666 & $-69: 02: 39.910$ & Obs. \\
\hline 800 & 32 & 25 & $05: 37: 45.929$ & $-69: 02: 39.910$ & Obs. \\
\hline 801 & 1 & 26 & $05: 39: 41.784$ & $-69: 02: 20.040$ & No Obs. \\
\hline 802 & 2 & 26 & 05:39:38.059 & $-69: 02: 19.930$ & Obs. \\
\hline 803 & 3 & 26 & $05: 39: 34.320$ & $-69: 02: 20.040$ & No Obs. \\
\hline 804 & 4 & 26 & $05: 39: 30.590$ & $-69: 02: 19.930$ & Obs. \\
\hline 805 & 5 & 26 & $05: 39: 26.856$ & $-69: 02: 20.040$ & No Obs. \\
\hline 806 & 6 & 26 & $05: 39: 23.110$ & $-69: 02: 19.930$ & Obs. \\
\hline 807 & 7 & 26 & 05:39:19.368 & $-69: 02: 20.040$ & No Obs. \\
\hline 808 & 8 & 26 & 05:39:15.629 & $-69: 02: 19.930$ & Obs. \\
\hline 809 & 9 & 26 & 05:39:11.880 & $-69: 02: 20.040$ & No Obs. \\
\hline 810 & 10 & 26 & 05:39:08.160 & $-69: 02: 19.930$ & Obs. \\
\hline 811 & 11 & 26 & 05:39:04.416 & $-69: 02: 20.040$ & Obs. (IG) \\
\hline 812 & 12 & 26 & 05:39:00.672 & $-69: 02: 20.040$ & Obs. (IG) \\
\hline 813 & 13 & 26 & 05:38:56.952 & $-69: 02: 20.040$ & Obs. (IG) \\
\hline 814 & 14 & 26 & $05: 38: 53.210$ & $-69: 02: 19.930$ & Obs. \\
\hline 815 & 15 & 26 & 05:38:49.464 & $-69: 02: 20.040$ & No Obs. \\
\hline 816 & 16 & 26 & $05: 38: 45.730$ & $-69: 02: 19.930$ & Obs. \\
\hline 817 & 17 & 26 & 05:38:41.976 & $-69: 02: 20.040$ & No Obs. \\
\hline 818 & 18 & 26 & 05:38:38.261 & $-69: 02: 19.930$ & Obs. \\
\hline 819 & 19 & 26 & $05: 38: 34.514$ & $-69: 02: 19.930$ & Obs. \\
\hline 820 & 20 & 26 & $05: 38: 30.780$ & $-69: 02: 19.930$ & Obs. \\
\hline 821 & 21 & 26 & $05: 38: 27.046$ & $-69: 02: 19.930$ & Obs. \\
\hline 822 & 22 & 26 & 05:38:23.309 & $-69: 02: 19.930$ & Obs. \\
\hline 823 & 23 & 26 & $05: 38: 19.565$ & $-69: 02: 19.860$ & Obs. \\
\hline 824 & 24 & 26 & 05:38:15.830 & $-69: 02: 19.930$ & Obs. \\
\hline 825 & 25 & 26 & 05:38:12.096 & $-69: 02: 19.860$ & Obs. \\
\hline 826 & 26 & 26 & 05:38:08.359 & $-69: 02: 19.930$ & Obs. \\
\hline 827 & 27 & 26 & $05: 38: 04.615$ & $-69: 02: 19.930$ & Obs. \\
\hline 828 & 28 & 26 & 05:38:00.881 & $-69: 02: 19.930$ & Obs. \\
\hline 829 & 29 & 26 & $05: 37: 57.146$ & $-69: 02: 19.930$ & Obs. \\
\hline 830 & 30 & 26 & $05: 37: 53.410$ & $-69: 02: 19.930$ & Obs. \\
\hline 831 & 31 & 26 & $05: 37: 49.666$ & $-69: 02: 19.860$ & Obs. \\
\hline 832 & 32 & 26 & $05: 37: 45.929$ & $-69: 02: 19.930$ & Obs. \\
\hline
\end{tabular}

Table A.1. continued.

\begin{tabular}{|c|c|c|c|c|c|}
\hline ID & $\begin{array}{c}X \\
\text { pix }\end{array}$ & $\begin{array}{c}Y \\
\text { pix }\end{array}$ & $\begin{array}{c}\text { RA (J2000) } \\
\text { h:m:s }\end{array}$ & $\begin{array}{c}\text { Dec (J2000) } \\
\text { d:m:s }\end{array}$ & Data \\
\hline 833 & 1 & 27 & $05: 39: 41.796$ & $-69: 01: 59.920$ & Obs. \\
\hline 834 & 2 & 7 & & 9.920 & bs. \\
\hline 835 & 3 & 7 & 25 & $-69:$ & Obs. \\
\hline 836 & 4 & 7 & 90 & $-69: 0$ & Obs. \\
\hline 837 & 5 & 7 & 46 & -69 : & Obs. \\
\hline 838 & 6 & 7 & 05:39: & $-69:($ & Obs. \\
\hline 839 & 7 & 27 & $05: 39: 1$ & $-69: 01:$ & Obs. \\
\hline 840 & 8 & 27 & 05:39: & $-69: 01: 59.920$ & Obs. \\
\hline 841 & 9 & 7 & 05:39: & 9.920 & Obs. \\
\hline 842 & 10 & 27 & .160 & -69 : & Obs. \\
\hline 843 & 11 & 7 & & -69 & Obs. \\
\hline 844 & 12 & 7 & & -69 & Obs. \\
\hline 845 & 13 & 27 & & -69 & Obs. \\
\hline 846 & 14 & 27 & & -69 & Obs. \\
\hline 847 & 15 & & & -69 & bs. \\
\hline 848 & 16 & & & -69 & bs. \\
\hline 849 & 17 & & & -69 & bs. \\
\hline 850 & 18 & & 51 & -69 & Obs. \\
\hline 851 & 19 & & 14 & -69 & Obs. \\
\hline 852 & 20 & 27 & 80 & -69 & Obs. \\
\hline 853 & 21 & 2 & $05:$ & $-69:$ & Obs. \\
\hline 854 & 22 & 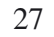 & 309 & -69 & Obs. \\
\hline 855 & 23 & & & -69 & Obs. \\
\hline 856 & 24 & & & & Obs. \\
\hline 857 & 25 & & & & Obs. \\
\hline 858 & 26 & & & & bs. \\
\hline 859 & 27 & & & & Obs. \\
\hline 860 & 28 & & & & Obs. \\
\hline 861 & 29 & & & & Obs. \\
\hline 862 & 30 & & & & Obs. \\
\hline 863 & 31 & & & & bs. \\
\hline 864 & 32 & & & & bs. \\
\hline 865 & 1 & & & & bs. \\
\hline 866 & 2 & & & - & bs. \\
\hline 867 & 3 & & & -69 & Obs. \\
\hline 868 & 4 & & & -69 & Obs. \\
\hline 869 & 5 & & & & bs. \\
\hline 870 & 6 & & & & bs. \\
\hline 871 & 7 & & & & bs. \\
\hline 872 & 8 & & & & bs. \\
\hline 873 & 9 & & & & bs. \\
\hline 874 & 10 & & & & bs. \\
\hline 875 & 11 & & & & bs. \\
\hline 876 & 12 & & & & os. \\
\hline 17 & 13 & & & & bs. \\
\hline 878 & 14 & & & & bs. \\
\hline 879 & 15 & & & -69 & bs. \\
\hline 880 & 16 & & 30 & -69 & bs. \\
\hline 881 & 17 & & & -69 & Obs. \\
\hline 882 & 18 & & & -69 & Obs. \\
\hline 8 & 19 & & & & Obs. \\
\hline 8 & 20 & & & & bs. \\
\hline 8 & 21 & & & & bs. \\
\hline 8 & 22 & & & $-c$ & bs. \\
\hline 8 & 23 & & & & Obs. \\
\hline 8 & 24 & 20 & & -65 & Obs. \\
\hline 889 & 25 & 28 & & & Obs. \\
\hline & 26 & & & & \\
\hline 891 & 27 & 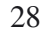 & & & Obs. \\
\hline 892 & 28 & 2 & & & \\
\hline 0 & 29 & & & & \\
\hline 0 & 30 & & & & \\
\hline 0 & 31 & & & $-69: 01: 39.860$ & \\
\hline 8 & 32 & & & & \\
\hline 897 & 1 & 29 & 05:39:41.796 & $-69: 01: 19.880$ & \\
\hline
\end{tabular}


Table A.1. continued.

\begin{tabular}{|c|c|c|c|c|c|}
\hline ID & $\begin{array}{c}X \\
\text { pix }\end{array}$ & $\begin{array}{c}Y \\
\text { pix }\end{array}$ & $\begin{array}{c}\text { RA (J2000) } \\
\text { h:m:s }\end{array}$ & $\begin{array}{c}\text { Dec (J2000) } \\
\text { d:m:s }\end{array}$ & Data \\
\hline 898 & 2 & 29 & $05: 39: 38.059$ & $-69: 01: 19.880$ & Obs. \\
\hline 899 & 3 & 29 & 05:39:34.325 & $-69: 01: 19.880$ & Obs. \\
\hline 900 & 4 & 29 & 05:39:30.590 & $-69: 01: 19.880$ & Obs. \\
\hline 901 & 5 & 29 & 05:39:26.846 & $-69: 01: 19.880$ & Obs. \\
\hline 902 & 6 & 29 & 05:39:23.110 & $-69: 01: 19.880$ & Obs. \\
\hline 903 & 7 & 29 & 05:39:19.366 & $-69: 01: 19.880$ & Obs. \\
\hline 904 & 8 & 29 & 05:39:15.629 & $-69: 01: 19.880$ & Obs. \\
\hline 905 & 9 & 29 & 05:39:11.894 & $-69: 01: 19.880$ & Obs. \\
\hline 906 & 10 & 29 & 05:39:08.160 & $-69: 01: 19.880$ & Obs. \\
\hline 907 & 11 & 29 & 05:39:04.421 & $-69: 01: 19.880$ & Obs. \\
\hline 908 & 12 & 29 & 05:39:00.679 & $-69: 01: 19.880$ & Obs. \\
\hline 909 & 13 & 29 & 05:38:56.945 & $-69: 01: 19.880$ & Obs. \\
\hline 910 & 14 & 29 & 05:38:53.210 & $-69: 01: 19.880$ & Obs. \\
\hline 911 & 15 & 29 & 05:38:49.466 & $-69: 01: 19.880$ & Obs. \\
\hline 912 & 16 & 29 & 05:38:45.730 & $-69: 01: 19.880$ & Obs. \\
\hline 913 & 17 & 29 & 05:38:41.995 & $-69: 01: 19.880$ & Obs. \\
\hline 914 & 18 & 29 & 05:38:38.261 & $-69: 01: 19.880$ & Obs. \\
\hline 915 & 19 & 29 & $05: 38: 34.514$ & $-69: 01: 19.880$ & Obs. \\
\hline 916 & 20 & 29 & 05:38:30.780 & $-69: 01: 19.880$ & Obs. \\
\hline 917 & 21 & 29 & 05:38:27.046 & $-69: 01: 19.880$ & Obs. \\
\hline 918 & 22 & 29 & 05:38:23.309 & $-69: 01: 19.880$ & Obs. \\
\hline 919 & 23 & 29 & 05:38:19.565 & $-69: 01: 19.880$ & Obs. \\
\hline 920 & 24 & 29 & 05:38:15.830 & $-69: 01: 19.880$ & Obs. \\
\hline 921 & 25 & 29 & 05:38:12.096 & $-69: 01: 19.880$ & Obs. \\
\hline 922 & 26 & 29 & 05:38:08.359 & $-69: 01: 19.880$ & Obs. \\
\hline 923 & 27 & 29 & 05:38:04.615 & $-69: 01: 19.880$ & Obs. \\
\hline 924 & 28 & 29 & 05:38:00.881 & $-69: 01: 19.880$ & Obs. \\
\hline 925 & 29 & 29 & 05:37:57.146 & $-69: 01: 19.880$ & Obs. \\
\hline 926 & 30 & 29 & 05:37:53.410 & $-69: 01: 19.880$ & Obs. \\
\hline 927 & 31 & 29 & 05:37:49.666 & $-69: 01: 19.880$ & Obs. \\
\hline 928 & 32 & 29 & 05:37:45.929 & $-69: 01: 19.880$ & Obs. \\
\hline 929 & 1 & 30 & 05:39:41.796 & $-69: 00: 59.940$ & Obs. \\
\hline 930 & 2 & 30 & 05:39:38.059 & $-69: 00: 59.940$ & Obs. \\
\hline 931 & 3 & 30 & 05:39:34.325 & $-69: 00: 59.940$ & Obs. \\
\hline 932 & 4 & 30 & 05:39:30.590 & $-69: 00: 59.940$ & Obs. \\
\hline 933 & 5 & 30 & $05: 39: 26.846$ & $-69: 00: 59.940$ & Obs. \\
\hline 934 & 6 & 30 & 05:39:23.110 & $-69: 00: 59.940$ & Obs. \\
\hline 935 & 7 & 30 & 05:39:19.366 & $-69: 00: 59.940$ & Obs. \\
\hline 936 & 8 & 30 & 05:39:15.629 & $-69: 00: 59.940$ & Obs. \\
\hline 937 & 9 & 30 & 05:39:11.894 & $-69: 00: 59.940$ & Obs. \\
\hline 938 & 10 & 30 & 05:39:08.160 & $-69: 00: 59.940$ & Obs. \\
\hline 939 & 11 & 30 & 05:39:04.421 & $-69: 00: 59.940$ & Obs. \\
\hline 940 & 12 & 30 & 05:39:00.679 & $-69: 00: 59.940$ & Obs. \\
\hline 941 & 13 & 30 & 05:38:56.945 & $-69: 00: 59.940$ & Obs. \\
\hline 942 & 14 & 30 & 05:38:53.210 & $-69: 00: 59.940$ & Obs. \\
\hline 943 & 15 & 30 & 05:38:49.466 & $-69: 00: 59.940$ & Obs. \\
\hline 944 & 16 & 30 & $05: 38: 45.730$ & $-69: 00: 59.940$ & Obs. \\
\hline 945 & 17 & 30 & 05:38:41.995 & $-69: 00: 59.940$ & Obs. \\
\hline 946 & 18 & 30 & 05:38:38.261 & $-69: 00: 59.940$ & Obs. \\
\hline 947 & 19 & 30 & $05: 38: 34.514$ & $-69: 00: 59.940$ & Obs. \\
\hline 948 & 20 & 30 & 05:38:30.780 & $-69: 00: 59.940$ & Obs. \\
\hline 949 & 21 & 30 & 05:38:27.046 & $-69: 00: 59.940$ & Obs. \\
\hline 950 & 22 & 30 & 05:38:23.309 & $-69: 00: 59.940$ & Obs. \\
\hline 951 & 23 & 30 & 05:38:19.560 & $-69: 00: 59.760$ & No Obs. \\
\hline 952 & 24 & 30 & 05:38:15.830 & $-69: 00: 59.940$ & Obs. \\
\hline 953 & 25 & 30 & 05:38:12.096 & $-69: 00: 59.760$ & No Obs. \\
\hline 954 & 26 & 30 & 05:38:08.359 & $-69: 00: 59.940$ & Obs. \\
\hline 955 & 27 & 30 & 05:38:04.615 & $-69: 00: 59.940$ & Obs. \\
\hline 956 & 28 & 30 & 05:38:00.881 & $-69: 00: 59.940$ & Obs. \\
\hline 957 & 29 & 30 & 05:37:57.146 & $-69: 00: 59.940$ & Obs. \\
\hline 958 & 30 & 30 & 05:37:53.410 & $-69: 00: 59.940$ & Obs. \\
\hline 959 & 31 & 30 & 05:37:49.656 & $-69: 00: 59.760$ & No Obs. \\
\hline 960 & 32 & 30 & 05:37:45.929 & $-69: 00: 59.940$ & No Obs. \\
\hline
\end{tabular}

Table A.2. Fiber positions of the irregular nebular grid.

\begin{tabular}{ccc}
\hline \hline ID & RA (J2000) & Dec $(\mathrm{J} 2000)$ \\
& h:m:s & d:m:s \\
\hline 1 & $05: 38: 45.040$ & $-69: 05: 09.600$ \\
2 & $05: 38: 47.970$ & $-69: 05: 03.600$ \\
3 & $05: 38: 51.330$ & $-69: 04: 59.900$ \\
4 & $05: 38: 44.980$ & $-69: 04: 50.600$ \\
5 & $05: 38: 43.050$ & $-69: 04: 31.900$ \\
6 & $05: 38: 55.000$ & $-69: 05: 01.600$ \\
7 & $05: 38: 36.380$ & $-69: 06: 21.200$ \\
8 & $05: 38: 34.580$ & $-69: 04: 56.900$ \\
9 & $05: 38: 32.340$ & $-69: 05: 16.900$ \\
10 & $05: 38: 37.320$ & $-69: 04: 37.900$ \\
11 & $05: 38: 31.340$ & $-69: 05: 40.200$ \\
12 & $05: 38: 42.430$ & $-69: 07: 04.300$ \\
13 & $05: 38: 54.010$ & $-69: 05: 32.600$ \\
14 & $05: 38: 33.200$ & $-69: 06: 52.600$ \\
15 & $05: 38: 38.070$ & $-69: 04: 20.200$ \\
16 & $05: 38: 43.420$ & $-69: 04: 15.900$ \\
17 & $05: 38: 59.170$ & $-69: 05: 08.900$ \\
18 & $05: 38: 31.670$ & $-69: 03: 44.200$ \\
19 & $05: 39: 02.540$ & $-69: 05: 20.800$ \\
20 & $05: 38: 37.270$ & $-69: 03: 36.900$ \\
21 & $05: 38: 38.380$ & $-69: 03: 57.600$ \\
22 & $05: 38: 35.400$ & $-69: 03: 48.900$ \\
23 & $05: 39: 02.040$ & $-69: 05: 42.200$ \\
24 & $05: 38: 56.070$ & $-69: 06: 34.200$ \\
25 & $05: 38: 56.810$ & $-69: 05: 46.200$ \\
26 & $05: 38: 31.700$ & $-69: 07: 21.200$ \\
27 & $05: 38: 09.570$ & $-69: 06: 21.300$ \\
28 & $05: 38: 53.370$ & $-69: 02: 00.900$ \\
29 & $05: 38: 45.040$ & $-69: 06: 54.900$ \\
30 & $05: 38: 30.700$ & $-69: 08: 09.600$ \\
31 & $05: 38: 40.060$ & $-69: 06: 33.600$ \\
32 & $05: 38: 49.970$ & $-69: 07: 12.900$ \\
33 & $05: 39: 09.370$ & $-69: 04: 32.800$ \\
34 & $05: 39: 25.560$ & $-69: 11: 38.200$ \\
35 & $05: 38: 27.820$ & $-69: 03: 08.500$ \\
36 & $05: 38: 19.120$ & $-69: 03: 12.500$ \\
37 & $05: 38: 16.870$ & $-69: 03: 23.800$ \\
38 & $05: 38: 41.300$ & $-69: 07: 34.600$ \\
39 & $05: 38: 57.050$ & $-69: 06: 05.700$ \\
40 & $05: 39: 11.490$ & $-69: 04: 42.100$ \\
41 & $05: 38: 11.680$ & $-69: 07: 30.400$ \\
42 & $05: 39: 05.900$ & $-69: 05: 24.800$ \\
43 & $05: 38: 10.160$ & $-69: 03: 22.100$ \\
44 & $05: 38: 10.050$ & $-69: 08: 01.100$ \\
45 & $05: 38: 18.390$ & $-69: 02: 18.100$ \\
46 & $05: 38: 21.790$ & $-69: 02: 59.200$ \\
47 & $05: 38: 48.180$ & $-69: 10: 12.200$ \\
48 & $05: 38: 29.070$ & $-69: 02: 44.200$ \\
49 & $05: 37: 59.150$ & $-69: 05: 25.200$ \\
50 & $05: 39: 27.080$ & $-69: 05: 41.900$ \\
51 & $05: 38: 57.530$ & $-69: 08: 19.500$ \\
52 & $05: 38: 38.430$ & $-69: 00: 39.100$ \\
53 & $05: 38: 03.900$ & $-69: 07: 06.600$ \\
54 & $05: 38: 38.490$ & $-69: 09: 53.600$ \\
55 & $05: 38: 08.530$ & $-69: 04: 00.000$ \\
56 & $05: 37: 50.440$ & $-69: 10: 12.600$ \\
58 & $05: 39: 04.910$ & $-69: 05: 52.800$ \\
59 & $05: 38: 30.050$ & $-69: 10: 11.600$ \\
60 & $05: 39: 35.030$ & $-69: 05: 02.100$ \\
61 & $05: 38: 23.970$ & $-69: 02: 30.200$ \\
62 & $05: 39: 24.330$ & $-69: 05: 15.300$ \\
63 & $05: 38: 05.520$ & $-69: 08: 01.900$ \\
64 & $05: 39: 24.110$ & $-69: 04: 40.700$ \\
\hline & & $-69: 06: 08.600$ \\
\hline 5 & \\
57 &
\end{tabular}


S. Torres-Flores et al.: Studying the kinematics of the giant star-forming region 30 Doradus. I.

Table A.2. continued.

\begin{tabular}{ccc}
\hline \hline ID & RA (J2000) & Dec (J2000) \\
& h:m:s:s & d:s \\
\hline 65 & $05: 39: 22.960$ & $-69: 07: 25.600$ \\
66 & $05: 39: 00.770$ & $-69: 08: 09.200$ \\
67 & $05: 39: 23.820$ & $-69: 07: 07.600$ \\
68 & $05: 38: 43.710$ & $-69: 00: 19.800$ \\
69 & $05: 38: 00.870$ & $-69: 06: 09.900$ \\
70 & $05: 39: 01.190$ & $-69: 02: 23.200$ \\
71 & $05: 39: 17.270$ & $-69: 01: 42.800$ \\
72 & $05: 39: 04.010$ & $-69: 07: 51.800$ \\
73 & $05: 39: 24.570$ & $-69: 04: 57.300$ \\
74 & $05: 37: 56.400$ & $-69: 05: 47.900$ \\
75 & $05: 39: 26.180$ & $-69: 06: 41.600$ \\
76 & $05: 37: 46.900$ & $-69: 07: 12.900$ \\
77 & $05: 39: 12.740$ & $-69: 07: 31.100$ \\
78 & $05: 38: 38.880$ & $-69: 08: 13.600$ \\
79 & $05: 39: 10.630$ & $-69: 08: 12.400$ \\
80 & $05: 39: 26.590$ & $-69: 05: 58.600$ \\
81 & $05: 38: 12.050$ & $-69: 02: 14.100$ \\
82 & $05: 38: 57.830$ & $-69: 02: 18.500$ \\
83 & $05: 38: 56.420$ & $-69: 10: 00.200$ \\
84 & $05: 39: 12.490$ & $-69: 07: 47.800$ \\
85 & $05: 37: 54.360$ & $-69: 07: 33.800$ \\
86 & $05: 37: 40.910$ & $-69: 06: 59.300$ \\
87 & $05: 37: 42.900$ & $-69: 10: 46.800$ \\
88 & $05: 38: 12.210$ & $-69: 08: 56.100$ \\
89 & $05: 38: 26.430$ & $-69: 09: 48.200$ \\
90 & $05: 39: 05.790$ & $-69: 02: 28.500$ \\
91 & $05: 37: 35.900$ & $-69: 06: 43.800$ \\
92 & $05: 38: 39.860$ & $-69: 01: 13.300$ \\
93 & $05: 38: 52.300$ & $-69: 09: 38.200$ \\
94 & $05: 38: 17.320$ & $-69: 09: 30.100$ \\
95 & $05: 39: 46.110$ & $-69: 07: 46.300$ \\
96 & $05: 37: 27.250$ & $-69: 06: 44.900$ \\
97 & $05: 37: 21.850$ & $-69: 06: 53.400$ \\
98 & $05: 37: 49.760$ & $-69: 10: 46.700$ \\
99 & $05: 38: 20.380$ & $-69: 13: 42.800$ \\
100 & $05: 38: 19.560$ & $-69: 10: 02.800$ \\
101 & $05: 39: 07.530$ & $-69: 02: 50.500$ \\
102 & $05: 39: 12.880$ & $-69: 08: 09.100$ \\
103 & $05: 39: 51.520$ & $-69: 06: 39.400$ \\
104 & $05: 39: 18.360$ & $-69: 08: 01.700$ \\
105 & $05: 38: 18.340$ & $-69: 12: 51.100$ \\
106 & $05: 37: 43.590$ & $-69: 10: 12.800$ \\
107 & $05: 38: 40.980$ & $-69: 13: 32.900$ \\
108 & $05: 38: 52.710$ & $-69: 12: 18.400$ \\
109 & $05: 38: 17.760$ & $-69: 11: 14.600$ \\
110 & $05: 38: 30.620$ & $-69: 13: 55.600$ \\
111 & $05: 39: 08.510$ & $-69: 08: 18.500$ \\
112 & $05: 38: 43.390$ & $-69: 17: 00.000$ \\
113 & $05: 37: 51.260$ & $-68: 58: 03.000$ \\
114 & $05: 37: 35.340$ & $-68: 58: 36.400$ \\
115 & $05: 37: 36.910$ & $-68: 58: 27.400$ \\
116 & $05: 37: 37.450$ & $-68: 58: 01.700$ \\
117 & $05: 37: 49.310$ & $-69: 16: 00.000$ \\
\hline & & \\
\hline
\end{tabular}

\section{References}

Bosch, G., Terlevich, E., \& Terlevich, R. 2009, AJ, 137, 3437

Chu, Y.-H., \& Kennicutt, R. C., Jr. 1994, Ap\&SS, 216, 253

Cox, P., \& Deharveng, L. 1983, A\&A, 117, 265

Crowther, P. A., Schnurr, O., Hirschi, R., et al. 2010, MNRAS, 408, 731

Danziger, I. J., Goss, W. M., Murdin, P., Clark, D. H., \& Boksenberg, A. 1981, MNRAS, 195, 33

Evans, C. J., Taylor, W. D., Hénault-Brunet, V., et al. 2011, A\&A, 530, A108

Hirata, R., \& Horaguchi, T. 1995, Atomic Spectral Line List, Kyoto Univ.

Lopez, L. A., Krumholz, M. R., Bolatto, A. D., Prochaska, J. X., \& Ramirez-Ruiz, E. 2011, ApJ, 731, 91

Lucke, P. B., \& Hodge, P. W. 1970, AJ, 75, 171

MacKenty, J. W., Maíz-Apellániz, J., Pickens, C. E., et al. 2000, AJ, 120, 3007

Maíz-Apellániz, J., \& Walborn, N. R. 2001, IAUS, 205, 222

Markwardt C. B. 2009, in Astronomical Data Analysis Software and Systems XVIII, eds. D. A. Bohlender, D. Durand, \& P. Dowler, ASPC Conf. Ser., 411, 251

Melnick, J., Tenorio-Tagle, G., \& Terlevich, R. 1999, MNRAS, 302, 677

Oey, M. S. 1996, ApJ, 467, 666

Pasquini, L., Avila, G., Blecha, A., et al. 2002, The Messenger, 110, 1

Pellegrini, E. W., Baldwin, J. A., \& Ferland, G. J. 2011, ApJ, 738, 34

Redman, M. P., Al-Mostafa, Z. A., Meaburn, J., \& Bryce, M. 2003, MNRAS, 344,741

Sana, H., de Koter, A., de Mink, S. E., et al. 2013, A\&A, 550, A107

Smith, M. G., \& Weedman, D. W. 1972, ApJ, 172, 307

Tenorio-Tagle, G., Muñoz-Tuñon, C., \& Cox, D. P. 1993, ApJ, 418, 767

Tenorio-Tagle, G., Muñoz-Tuñon, C., \& Cid-Fernandes, R. 1996, ApJ, 456, 264

Torres-Flores, S., Barbá, R., Apellániz, J. M., Rubio, M., \& Bosch, G. 2011, BAAA, 54, 243

Townsley, L. K., Broos, P. S., Feigelson, E. D., Garmire, G. P., \& Getman, K. V. 2006a, AJ, 131, 2164

Townsley, L. K., Broos, P. S., Feigelson, E. D., et al. 2006b, AJ, 131, 2140

Walborn, N. R. 1991, IAUS, 148, 145

Walborn, N. R., Maíz-Apellániz, J., \& Barbá, R. H. 2002, AJ, 124, 1601

Wang, Q. D. 1999, ApJ, 510, L139

Wang, Q., \& Helfand, D. J. 1991, ApJ, 370, 541

Weaver, R., McCray, R., Castor, J., Shapiro, P., \& Moore, R. 1977, ApJ, 218, 377 